Guide to Nondestructive Assay Standards:

Preparation Criteria, Availability, and

Practical Considerations
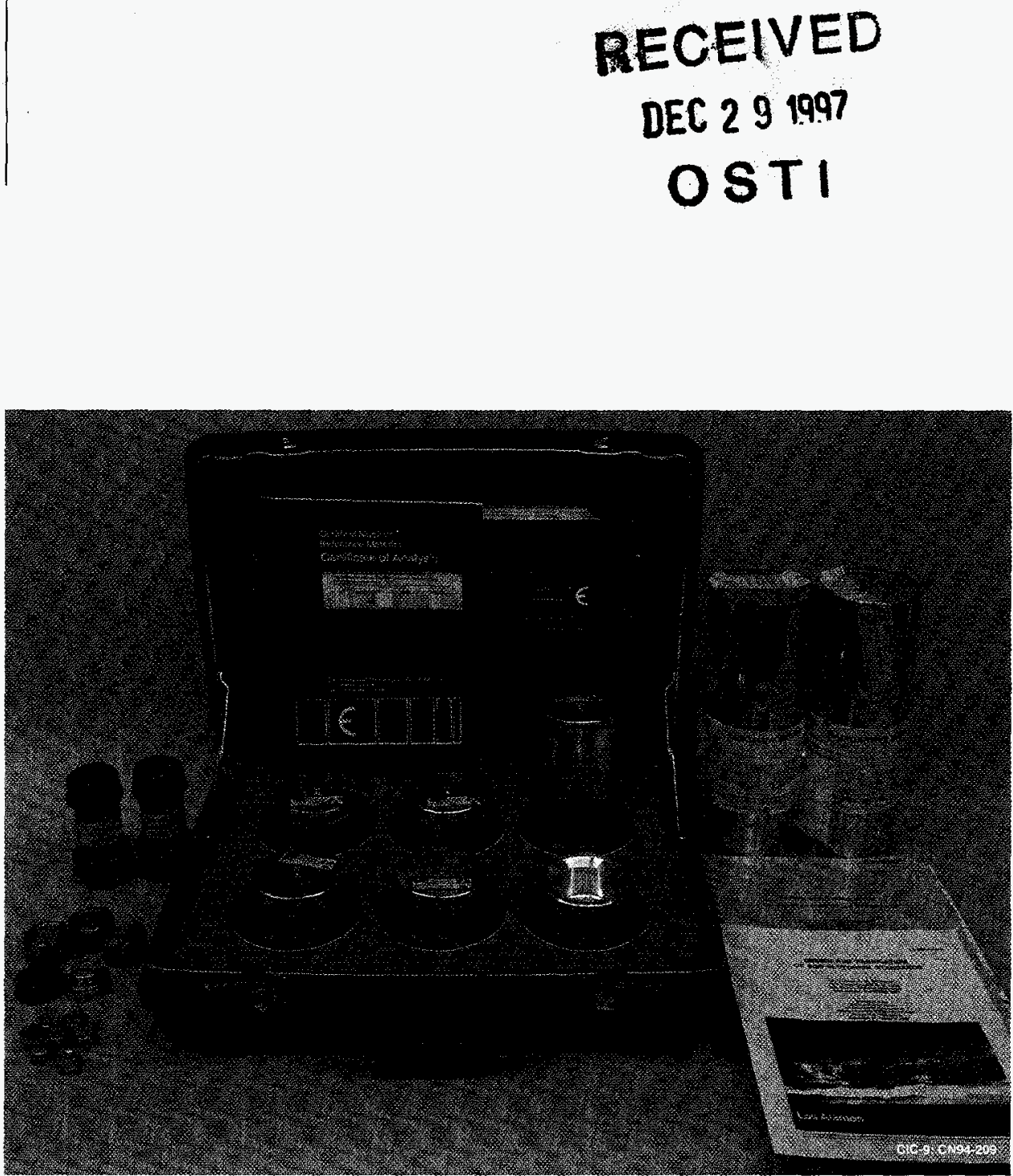
This work was supported by the US Department of Energy,

Office of Nonproliferation and National Security,

Office of Safeguards and Security.

\section{Edited by Jeff Skiby, Group CIC-1 \\ Prepared by Genara Jaramillo, Group NIS-5}

Front cover: Starting from the center and continuing around clockwise, European Community Certified Nucelar Reference Material 171/New Brunswick Laboratory Certified Reference Material (CRM) 969 (briefcase); Segmented Gamma-Ray Scanning Standards (tall stainless steel containers); Plutonium Isotopic Determination Intercomparison Exercise ( PIDIE) Standards; Central Bureau of Nuclear Measurements Nuclear Reference Material 271 (lower left of briefcase); and CRM 136, 137, and 138, formerly National Bureau of Standards 946, 947, and 948 .

An Affirmative Action/Equal Opportunity Employer

This report was prepared as an account of work sponsored by an agency of the United States Government. Neither The Regents of the University of California, the United States Government nor any agency thereof, nor any of their employees, makes any warranty, express or implied, or assumes any legal liability or responsibility for the accuracy, completeness, or usefuiness of any information, apparatus, product, or process disclosed, or represents that its use would not infringe privately owned rights. Reference herein to any specific commercial product, process, or service by trade name, trademark, manufacturer, or otherwise, does not necessarily constitute or imply its endorsement, recommendation, or favoring by The Regents of the University of California, the United States Government, or any agency thereof. The views and opinions of authors expressed herein do not necessarily state or reflect those of The Regents of the University of California, the United States Government, or any agency thereof. Los Alamos National Laboratory strongly supports academic freedom and a researcher's right to publish; as an institution, however, the Laboratory does not endorse the viewpoint of a publication or guarantee its technical correctness. 


\section{DISCLADIER}

Portions of this docoment may be illegible in electronic image prodnets. Inages are produced from the best arailable original document. 
Guide to Nondestructive Assay Standards:

Preparation Criteria, Availability, and

Practical Considerations

S. -T. Hsue

J. E. Stewart

T. E. Sampson

G. Butler

C. R. Rudy

P. M. Rinard 


\section{CONTENTS}

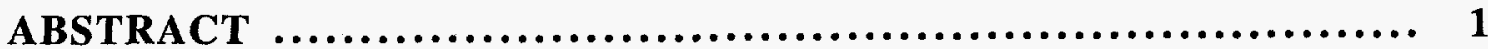

EXECUTIVE SUMMARY $\ldots \ldots \ldots \ldots \ldots \ldots \ldots \ldots \ldots \ldots \ldots \ldots \ldots \ldots \ldots \ldots \ldots \ldots$

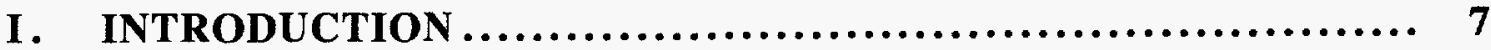

A . Motivation............................................... 7

B . Practical Guidance................................... 8

C. Definitions ........................................... 9

1. Standards ...................................................... 9

2. Certified Reference Material ...................................... 9

3. Working Reference Material.................................... 10

References............................................... 10

II. OVERVIEW: GAMMA-RAY TECHNIQUES................. 13

A . Plutonium Isotopic Measurements $\ldots \ldots \ldots \ldots \ldots \ldots \ldots \ldots \ldots \ldots \ldots$

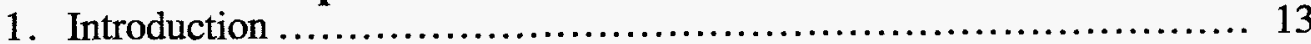

2. Measurement Principles................................................. 13

3. Use of Standards ................................................. 14

4. Examples of Standards............................................. 18

a. Isotopic Reference Materials for NDA........................ 18

b. Isotopic Reference Materials for Mass Spectrometry ............... 18

c. Working Standards for Isotopic Analysis....................... 19

d. Segmented Gamma Scanner (tall stainless steel containers) .......... 19

e. EC Certified Nuclear Reference Material 171/NBL CRM 969 (briefcase) ....................................... 19

f. CRM 136, 137, 138, Formerly NBS 946, 947, 948

(contained in pipe nipples) .................................. 19

g. CBNM Nuclear Reference Material $271 \ldots \ldots \ldots \ldots \ldots \ldots \ldots \ldots \ldots \ldots . \ldots \ldots$

5. Calorimetry Exchange Program.................................... 20

6. Practical Considerations .......................................... 21

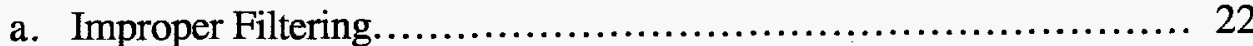

b. Improper Count Rates ...................................... 22

c. Poor Quality Detector........................................ 22

d. Unanticipated Isotope Interferences............................ 22

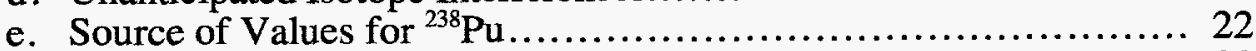

f. Am-241 Separation ........................................... 22

g. Units of Reported Results..................................... 23

Conclusions.$\ldots \ldots \ldots \ldots \ldots \ldots \ldots \ldots \ldots \ldots \ldots \ldots \ldots \ldots \ldots \ldots \ldots \ldots \ldots \ldots \ldots$

References............................................. 23

B . Uranium Enrichment Measurements..................... 25

1. Measurement Principles......................................... 25

2. Physical Criteria for Standards........................................ 26

3. The "quasi-infinite" Sample Size ............................... 26 
4. Preparation and Examples of Standards .......................... 28

References............................................... 29

C. Solutions $\ldots \ldots \ldots \ldots \ldots \ldots \ldots \ldots \ldots \ldots \ldots \ldots \ldots \ldots \ldots \ldots \ldots \ldots \ldots \ldots \ldots \ldots$

1. Measurement Principles............................................ 30

2. Preparation of Standards......................................... 31

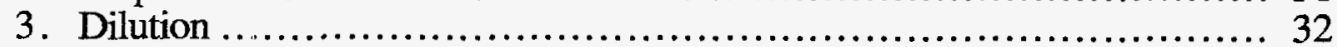

4. General Comments .............................................. 33

References................................................. 33

D. Segmented Gamma Scanning $\ldots \ldots \ldots \ldots \ldots \ldots \ldots \ldots \ldots \ldots \ldots \ldots \ldots$

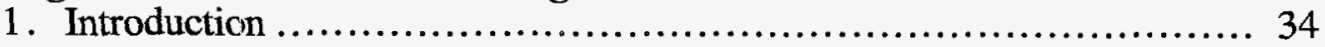

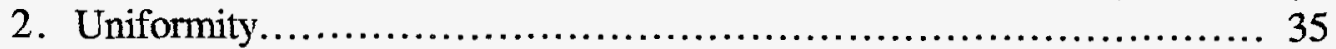

3. Transmission........................................................ 35

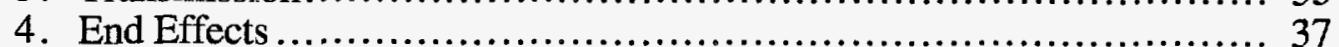

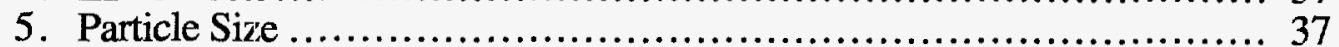

6. Typical Examples of SGS Standards............................... 39

References................................................. 40

III. OVERVIEW: NEUTRON AND CALORIMETER

MEASUREMENTS...................................... 41

A. Neutron Techniques $. . \ldots \ldots \ldots \ldots \ldots \ldots \ldots \ldots \ldots \ldots \ldots \ldots \ldots \ldots \ldots . \ldots 1$

1. Bulk Measurements of Uranium and Plutonium Masses ................ 41

a. Thermal Neutron Coincidence Counting ......................... 41

b. Thermal Neutron Multiplicity Counting ........................... 46

References............................................. 47

2. Californium Shuffler $\ldots \ldots \ldots \ldots \ldots \ldots \ldots \ldots \ldots \ldots \ldots \ldots \ldots \ldots \ldots . . \ldots 8$

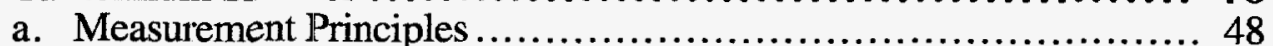

b. Physical Criteria for Standards .................................... 48

c. Preparation of Practical Standards ................................. 49

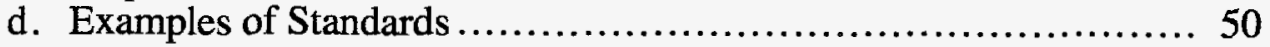

References...$\ldots \ldots \ldots \ldots \ldots \ldots \ldots \ldots \ldots \ldots \ldots \ldots \ldots \ldots \ldots \ldots \ldots \ldots \ldots 1$

B . Calorimetry $\ldots \ldots \ldots \ldots \ldots \ldots \ldots \ldots \ldots \ldots \ldots \ldots \ldots \ldots \ldots \ldots \ldots \ldots \ldots \ldots \ldots$

1. Principles ............................................................ 53

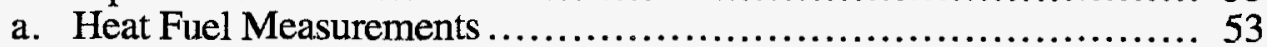

b. Specific Power and SNM Mass Determination..................... 54

2. Standard Requirements ............................................ 55

a. Radioactive Heat Standards.................................... 55

b. Electrical Standards ............................................. 58

References $\ldots \ldots \ldots \ldots \ldots \ldots \ldots \ldots \ldots \ldots \ldots \ldots \ldots \ldots \ldots \ldots \ldots \ldots \ldots \ldots \ldots$

IV. CURRENTLY AVAILABLE NONDESTRUCTIVE ASSAY

STANDARDS........................................ 61

A. Certified Reference Materials..........................61 


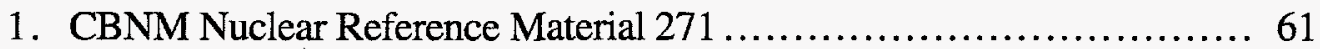

2. Certified Nuclear Reference Material EC NRM 171/NBL

CRM 969.

B . Working Reference Material...............................63

1. Performance Laboratory Standards .................................. 65

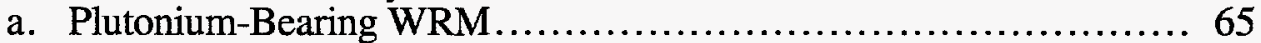

b. MOX WRM................................................ 66

2. PIDIE Plutonium Isotopic Standards............................... 66

3. Los Alamos Fabricated WRM.................................... 67

4. Segmented Gamma Scanning Plutonium Can Standard ................ 67

5. SGS Uranium Drum Standard ..................................... 70

6. Active Well Coincidence Counter (AWCC) Uranium Standard........... 71

7. Performance Demonstration Program (PDP) Plutonium Working Reference Material............................................... 73

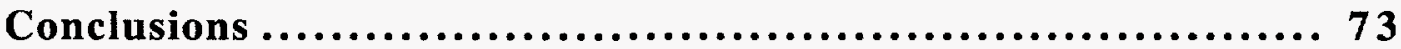

References................................................. 74

V. PREPARATION PROCEDURE AND TRACEABILITY .......... 77

A. Planning............................................. 77

B . Preparation.......................................... 77

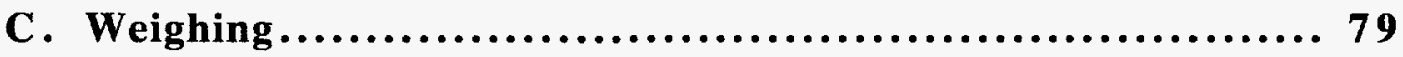

D. Container and Packaging................................80

E. Sampling and Characterization $. . \ldots \ldots \ldots \ldots \ldots \ldots \ldots \ldots \ldots \ldots, 80$

F. Verification .......................................... 82

Conclusion................................................. 83

References................................................ 83

VI. ALTERNATIVES TO STANDARDS........................ 85

A. Detector Family Techniques-Cross Calibration............... 85

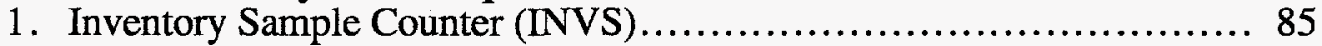

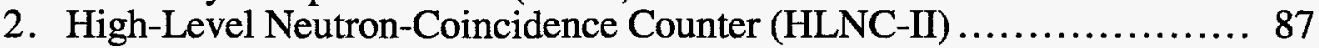

3. Active-Well Coincidence Counter ................................. 88

4. Uranium Neutron Coincidence Collar.............................. 90

5. Cross-Calibration Summary....................................... 90

B . Use Of Monte Carlo Simulations To Minimize

The Required Number Of Standards $. . . \ldots \ldots \ldots \ldots \ldots \ldots \ldots \ldots, 91$

1. Fast Breeder Reactor Mixed-Oxide Fuel Elements ................... 91

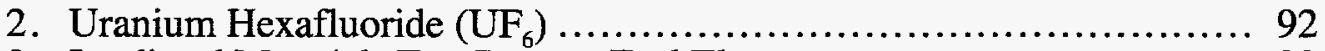

3. Irradiated Materials Test Reactor Fuel Elements .................... 92 
C. Use Of Calorimetry/Gamma-Ray Spectroscopy To Reduce The Need For Destructive Analysis ............... 93

References................................................ 93

VII. QUALITY ASSURANCE OF STANDARDS ...................95

A. Calorimetry Exchange Program $\ldots \ldots \ldots \ldots \ldots \ldots \ldots \ldots \ldots \ldots \ldots, 95$

B . Calibration Using Calorimetry/Gamma-Ray Assay .......... 96

C. Standards Recertification ................................. 99

References................................................. 100

VIII. RECOMMENDATIONS.................................... 101

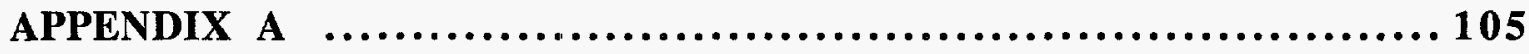




\section{ACRONYMS}

\begin{tabular}{|c|c|}
\hline AERE & $\begin{array}{l}\text { Atomic Energy Research } \\
\text { Establishment }\end{array}$ \\
\hline AFO & Albuquerque Field Office \\
\hline $\mathbf{A L}$ & analytical laboratory \\
\hline $\mathbf{A N C C}$ & $\begin{array}{l}\text { active neutron coincidence } \\
\text { counting }\end{array}$ \\
\hline ASTM & $\begin{array}{l}\text { American Society for Testing } \\
\text { and Materials }\end{array}$ \\
\hline $\mathbf{A W C C}$ & $\begin{array}{l}\text { Active Well Coincidence } \\
\text { Counter }\end{array}$ \\
\hline BWR & boiling water reactor \\
\hline CALEX & $\begin{array}{l}\text { Calorimetry Sample } \\
\text { Exchange }\end{array}$ \\
\hline CBNM & $\begin{array}{l}\text { Central Bureau of Nuclear } \\
\text { Measurements }\end{array}$ \\
\hline CEC & $\begin{array}{l}\text { Commission of European } \\
\text { Communities }\end{array}$ \\
\hline CRM & certified reference material \\
\hline CSL & $\begin{array}{l}\text { chemistry standard } \\
\text { laboratory }\end{array}$ \\
\hline $\mathbf{D A}$ & $\begin{array}{l}\text { destructive analysis or } \\
\text { chemical analysis }\end{array}$ \\
\hline DE & diatomaceous earth \\
\hline DOE & Department of Energy \\
\hline DVM & digital voltmeter \\
\hline FFTF & Fast Flux Test Facility \\
\hline FBR & fast breeder reactor \\
\hline HEU & highly enriched uranium \\
\hline HLNC-II & $\begin{array}{l}\text { High-Level Neutron } \\
\text { Coincidence Counter }\end{array}$ \\
\hline IAEA & $\begin{array}{l}\text { International Atomic Energy } \\
\text { Agency }\end{array}$ \\
\hline INVS & inventory sample counter \\
\hline IRMM & $\begin{array}{l}\text { Institute for Reference } \\
\text { Materials and } \\
\text { Measurements }\end{array}$ \\
\hline KED & $\begin{array}{l}\text { K-Edge absorption } \\
\text { densitometry }\end{array}$ \\
\hline LLNL & $\begin{array}{l}\text { Lawrence Livermore } \\
\text { National Laboratory }\end{array}$ \\
\hline LOI & loss-on-ignition \\
\hline $\mathbf{L W R}$ & light-water reactor \\
\hline MC & mixing container \\
\hline MC\&A & $\begin{array}{l}\text { materials control and } \\
\text { accounting }\end{array}$ \\
\hline MEC & $\begin{array}{l}\text { Mound Electrical Calibration } \\
\text { Heater }\end{array}$ \\
\hline
\end{tabular}

\begin{tabular}{|c|c|}
\hline MOX & mixed oxide \\
\hline MS & mass spectrometry \\
\hline MTR & Materials Testing Reactor \\
\hline NBL & New Brunswick Laboratory \\
\hline NBS & National Bureau of Standards \\
\hline $\mathbf{N C C}$ & oincidence counting \\
\hline NDA & nondestructive assay \\
\hline NDT & nondestructive testing \\
\hline NIST & $\begin{array}{l}\text { National Institute of Science } \\
\text { and Technology }\end{array}$ \\
\hline PAN & passive and active neutron \\
\hline PDP & $\begin{array}{l}\text { Performance Demonstration } \\
\text { Program }\end{array}$ \\
\hline PERLA & PERformance LAboratory \\
\hline PFPF & $\begin{array}{l}\text { Plutonium Fuel Production } \\
\text { Facility }\end{array}$ \\
\hline IDIE & Plutonium Isotopic \\
\hline & Determination \\
\hline & Intercomparison Exercise \\
\hline $\mathrm{C}$ & $\begin{array}{l}\text { passive neutron coincidence } \\
\text { counting }\end{array}$ \\
\hline & pressurized-water-reactor \\
\hline RRFC & Research Reactor Fuel Counter \\
\hline SA & solution assay instrument \\
\hline SALF & $\begin{array}{l}\text { Safeguards Analytical } \\
\text { Laboratory Evaluation }\end{array}$ \\
\hline SGS & $\begin{array}{l}\text { segmented gamma-ray scanner } \\
\text { special nuclear materials }\end{array}$ \\
\hline $\mathbf{S} / \mathbf{R}$ & shipper/receiver \\
\hline SRM & standard reference material \\
\hline & Savannah River Site \\
\hline & standard vial \\
\hline$r$ & $\begin{array}{l}\text { thermal neutron coincidence } \\
\text { counter }\end{array}$ \\
\hline TN & $\begin{array}{l}\text { thermal neutron multiplicity } \\
\text { counter }\end{array}$ \\
\hline $\mathbf{T}$ & trans \\
\hline UFBC & $\begin{array}{l}\text { Universal Fast Breeder Reactor } \\
\text { Subassembly Counter }\end{array}$ \\
\hline UN & $\begin{array}{l}\text { Uranium Neutron Coincidence } \\
\text { Collar }\end{array}$ \\
\hline & Unite \\
\hline & workin \\
\hline $\mathbf{w}$ & $\begin{array}{l}\text { Westinghouse Savannah Rive } \\
\text { Site }\end{array}$ \\
\hline & $\pi$ \\
\hline
\end{tabular}




\title{
GUIDE TO NONDESTRUCTIVE ASSAY STANDARDS: PREPARATION CRITERIA, AVAILABILITY, AND PRACTICAL CONSIDERATIONS*
}

\author{
S.-T. Hsue, J. E. Stewart, T. E. Sampson, \\ G. W. Butler, C. R. Rudy, and P. M. Rinard
}

\begin{abstract}
For certification and measurement control, nondestructive assay (NDA) instruments and methods used for verification measurements of special nuclear materials (SNMs) require calibrations based on certified reference materials (CRMs), or working reference materials (WRMs), traceable to the national system of measurements, and adequately characteristic of the unknowns. The Department of Energy Office of Safeguards and Security is sponsoring production of a comprehensive guide to preparation of NDA standards. The scope of the report includes preparation criteria, current availability of CRMs and WRMs, practical considerations for preparation and characterization, and an extensive bibliography.

In preparing the report, based primarily on experience at Los Alamos, we have found that standards preparation is highly dependent on the particular NDA method being applied. We therefore include sections that contain information specific to commonly used neutron and gamma-ray NDA techniques.

We also present approaches that are alternatives to, or minimize requirements for physical standards. In this section, we describe instrument cross-calibration, where one member of a family of nearly identical detectors is carefully calibrated with WRMs (defining the calibration curve shape), and other detectors are normalized to the reference detector, using a single WRM, or an isotopic source. Another alternative, that is becoming increasingly viable, is the use of Monte Carlo simulations to define shape, and a single WRM, or isotopic source for normalization. Another cost- and effort-saving approach is characterize WRMs with the combination of calorimetry and plutonium isotopics ( $\mathrm{Cal} / \mathrm{Iso}$ ). In many practical cases, $\mathrm{Cal} / \mathrm{Iso}$ values are more accurate than can be obtained with destructive analysis and weighing.

The guide is tailored to a wide audience, US DOE facilities, although considerable interest has been shown from those concerned with materials control and accountability in Russia, Europe, Japan and South America.
\end{abstract}





\section{EXECUTIVE SUMMARY}

As the role of nondestructive assay (NDA) techniques has grown in the implementation of material control and accounting (MC\&A) systems for domestic US and international safeguards at nuclear facilities, so has the importance of physical standards for instrument calibration and measurement control. Most NDA techniques rely on physical standards for calibration.

Through calibration measurements of physical standards, an absolute or relative relationship is established between instrument response and the masses or ratios of SNM nuclides known to be present. Physical standards are also routinely used to test, verify, and normalize instrument response.

Physical properties of standards are considered to be known absolutely through accepted systems of fabrication, analysis, documentation, and control. The "true" values of standards parameters are usually based on methods of destructive analyses (DA) and must be as free of bias as is practicable, depending on the NDA method in question.

For optimum use of physical standards, physical properties must match those of unknowns sufficiently so as to not significantly alter the observed NDA response. Frequently, success is obtained when physical standards have known, correctable deviations in properties, compared with unknowns.

Physical standards for NDA, then, are a necessary part of the successful application of any NDA technique, regardless of the number or variety required. While many papers and books have been written describing development and application of NDA techniques, very few publications have been devoted exclusively to NDA standards. This report is intended as a review of available NDA standards and the fundamental physical principles involved in their preparation.

The Introduction (Chapter I) presents the motivation for preparing the report, the rationale for the emphasis on practical guidance and some fundamental definitions for standards. Next we discuss the individual NDA techniques most frequently used in the US and European facilities with emphasis on standards that are applicable to each technique. For each NDA method, a description of the physical principles of the measurement, guidelines for preparation of standards, availability of standards, and practical considerations are given in Chapters II and III. In Chapter IV we discuss the certified reference (CRM) and working reference material (WRM) standards that are currently available in the US and Europe. Chapter V is a generic description of the standards preparation procedures and traceability to the national system of measurements. In Chapter VI, we discuss methods to maximally utilize existing standards and alternatives to standards that are less costly and timeconsuming. Chapter VII describes quality assurance of standards through the calorimetry exchange program, calibration using the combination calorimetry/gamma-ray assay and standards recertification. The last chapter (Chapter VIII) gives six recommendations and the rationale for each. These recommendations are repeated here, as an aid to the reader. 


\section{RECOMMENDATIONS}

1. Establish, within the United States, a resource center (such as PERLA in the EEC) housing an extensive collection of wellcharacterized NDA standards and where laboratories exist in which NDA systems are characterized, calibrated, and used for training.

The US has no dedicated center where users can bring their NDA instruments for evaluation and calibration. We recommend establishing, in the US, a resource center such as the Safeguards Performance Laboratory (PERLA) in the European Economic Community (EEC).

\section{Request that NBL/IRMM develop several CRMs.}

Several CRMs should be produced that would be very useful to the user community. Because of the difficulty in preparing CRMs, they should be selected carefully in conjunction with the development effort to reduce the number of standards required to calibrate NDA systems. If New Brunswick Laboratory (NBL) and the European Institute for Reference Materials and Measurements (IRMM) have difficulty in fabricating some of these CRMs, national laboratories can provide support. The plutonium-bearing CRMs may be issued in limited sets because of the difficulty in shipping. Two needs stand out: a pure plutonium metal standards set and a low-density standards set.

\section{Develop bulk-plutonium WRMs}

Several WRMs should be produced that would be very useful to the user community at the resource facility. These items should not be shipped from site to site. Three needs have been identified: an impure oxide set, a pure plutonium metal set, and a MOX set and a wet plutonium oxide set.

4. Establish the combination of calorimetry and plutonium gamma spectrometry as an acceptable, routine method to certify plutonium-bearing WRMs.

The combination of calorimetry and plutonium gamma spectrometry is a powerful approach for characterization of plutonium-bearing WRMs. The combination is less precise and has larger bias than chemical analysis, but the combination is cheaper and more timely. With sufficient counting time, precision and bias of better than $0.3 \%$ can be achieved for homogeneous and relatively pure plutonium samples. This is certainly sufficient for waste assay systems where the precision and bias are in the several percent range. This is probably adequate for certifying secondary WRMs. 
5. Publish a reference manual on the standards (RMs) that cannot and should not be shipped from site to site.

Some NDA standards, because of physical size, cannot be shipped from site to site. Also, some low-level waste standards that are used to flag disposable waste at the $100 \mathrm{nCi} / \mathrm{g}$ level, should not be shipped to other sites because of ease of preparation. It will be very useful to publish a practical guide to illustrate how these standards are made.

6. Establish the calculational approach as an acceptable, routine method of NDA instrument calibration.

Some standards cannot be characterized without being partially or completely destroyed at great expense. Examples of this are fresh and spent reactor fuelelements. 


\section{INTRODUCTION}

\section{A. Motivation}

Material control and accounting (MC\&A) systems are designed to keep track of special nuclear materials (SNM) in any accountable process, and they are based on quantitative measurements that are used to form material balances within that process. Accordingly, our ability to use material balances to detect significant losses from the process depends greatly on accurate measurements. In the last decade, nondestructive assay (NDA) techniques have become important analytical tools for the quantitative determination of SNM. NDA has the advantage that it is often cheaper and more timely than destructive analysis (DA), requires less training of the operator, and does not generate any hazardous or radioactive waste. In some cases, such as scrap and waste from chemical or physical processes involving SNM wherein the sample is often very non-homogeneous, NDA may be the only method for determining the SNM content with reasonable accuracy. A detailed discussion of the various NDA techniques can be found in Passive Nondestructive Assay of Nuclear Materials published by the Nuclear Regulatory Commission in 1991.

Most NDA techniques rely on standards for calibration. A good calibration defines an absolute or relative relationship between the NDA instrument response and masses or ratios of SNM nuclides known to be present. The calibration takes into account the detection efficiency, any collimator between the sample and detector, all absorbers, and any other factor that will affect the response of the system. NDA standards, therefore, are a crucial part of any NDA technique, regardless of the number or variety required. While many papers and books have discussed NDA techniques, very few publications have been devoted exclusively to NDA standards. This report is intended as a review of the available NDA standards and the fundamental physical principles involved in their preparation. We have also included a bibliography on NDA standards in the Appendix.

NDA standards are generally of a physical size, SNM mass, and chemical form that is applicable to the NDA technique being calibrated, but the standards do not have to match exactly the type of material being measured. There is a general impression that the standards need to match the samples in all aspects of chemical composition; this is overly restrictive and would require many different standards. NDA standards should have properties that represent the materials being measured in all characteristics that affect the NDA measurement. For NDA standards the emphasis should be placed on calibrating the technique. For example, plutonium oxide standards for neutron coincidence counting need to contain a minimum of the impurities that contribute to the $(\alpha, n)$ reaction, whereas standards that contain a substantial amount of such impurities are perfectly adequate for neutron multiplicity counting. Understanding the physical principles of all the NDA techniques offers the opportunity to minimize the number of sets of standards required. Standards with the best pedigree (high-quality documentation that provides traceability to the national system of measurements) will not necessarily be suitable for the calibration of a technique that does not represent in some significant way the material in question. This report explains the important factors of the standards required for each of the NDA techniques discussed. 
First we will discuss the NDA techniques that are most frequently used in the United States (US) and European facilities with the emphasis on the standards that are applicable to each technique. We will not discuss the emerging NDA technologies, such as tomographic gamma scanning, because these newer techniques are still in development.

The preparation of an NDA standard is expensive and time-consuming. If a set of standards is prepared incorrectly, it is a waste of both time and money; in addition, SNM waste is generated and it must be disposed of or recovered. Therefore the fabrication of standards must be planned very carefully. Clearly the physical properties of SNM (nuclide mass, composition, distribution, and matrix) significant to NDA instrument response must be known precisely throughout the fabrication procedure and sample characterization. The examples in this report show the amount of care and thought that have gone into the preparation of each set of working reference material (WRM) standards and should be a useful guide to those who need to prepare standards. We will also discuss how well the standards need to be prepared and characterized. In some cases, such as waste assays, where the precision and bias requirements are less stringent than for input or output samples, highly accurate standards of good pedigree are perhaps not necessary. We will discuss an alternative method of certifying waste assay standards that is easy to accomplish and therefore less costly.

\section{B . Practical Guidance}

We would like to emphasize that this report is intended to serve as a practical guide to the NDA user community. In Chapter IV, in addition to the NDA techniques, we discuss the certified reference material (CRM) and WRM standards that are currently available. Only two sets of NDA CRMs are available in the US and Europe. While it is not possible to discuss every set of WRM that exists, we will select certain well-prepared and documented WRM sets that best represent the wide variety of WRM standards that have been prepared. In Chapter $\mathrm{V}$ we address standards preparation methodology by summarizing the general underlying principles of the preparation of standards. In this chapter we also describe how these standards can be traced back to a chemical analysis. Chemical analysis (destructive analysis, or DA) has existed much longer than NDA and the traceability of DA to CRM standards is better established and accepted by the user communities. DA provides the link that allows NDA measurements to be traceable to the national system of measurements.

One fact stands out from experience with NDA calibration: if the assay technique has a linear response as a function of SNM content, then the number of standards needed for calibration is small, ranging from three to five. In these systems, a low-mass standard is not very useful because the counting statistics of such a standard are poor in a normal counting time. If the assay technique has a nonlinear response, such as the Active Well Coincidence Counter (AWCC), then it is crucial to have many standards covering the assay range to adequately define the nonlinear curve.

While it is desirable to have many sets of CRM standards for an NDA system, in practice they are not apt to be available in a timely manner. Some of the requirements for WRMs are mandated by Department of Energy (DOE) regulations and must be followed closely. Therefore facilities need to be conversant in the preparation of WRMs. This report is intended to assist the staff of nuclear facilities in understanding how to prepare 
WRMs. There are also many users of NDA instruments who are routinely confronted with calibration requirements. This report will help them in understanding what standards are available and how some other facilities have dealt with the standards issue.

Some of the standards are hard to fabricate and difficult to store. A typical example of this is irradiated spent nuclear fuel. These samples are highly radioactive, hard to characterize, and difficult to store. When a spent-fuel element is chemically characterized, the standard is destroyed in the process. In these cases, the alternative to using a standard is to certify a calculational method. An example of this will be given in Chapter VI whereby Monte Carlo calculations were successfully used to simulate mixed oxide (MOX) fuel elements. The calculational method can also be used to reduce the cost of fabricating NDA standards.

After a set of standards has been prepared, it is important to have a program to assure the quality of the standards. As we point out in Chapter VII, this can be done by using NDA to reverify standards. It can also be done by round robin sample exchange or other material exchange programs. We will also discuss the calorimetry exchange (CALEX) program in the US in some detail.

Finally we discuss our recommendations for future directions of research and development on NDA standards that can save the NDA user community money and effort. One of the recommendations is to establish the acceptance of NDA techniques to certify standards. Another recommendation is to achieve better acceptance of calculational approaches for the design and also for the certification of standards.

\section{Definitions}

1. Standards. Standards can be divided into primary and secondary categories. Primary NDA standards have a good pedigree, uniform distribution of SNM, good chemical characterization traceable to chemical CRMs, and consistent verification measurements. These standards can be used with confidence for the technique in use. NDA CRM standards are always primary standards for the calibration of an assay technique.

Secondary standards have one or more problems with their pedigree. The sample may not have a uniform distribution of SNM, or the verification measurement may detect some inconsistency with the primary standards. In these cases, some adjustments may have to be made to the accepted values of the standard, making it a secondary standard.

2. Certified Reference Material. CRM standards prepared from nuclear materials are typically of high purity and have good chemical stability or reproducible stoichiometry. They are certified using the most accurate and precise measurement methods available, often with more than one laboratory involved in making certification measurements. CRMs are generally used on a national or international level, and they are at the top of the metrological hierarchy of reference materials.

A CRM standard has one or more property values certified by a technically valid procedure and is always accompanied by or traceable to a certificate or other documentation issued by a certifying body. In the US the certifying body for chemical composition, isotopic composition, and NDA measurements is the New Brunswick Laboratory (NBL) of DOE. In Europe, the certifying body is the Central Bureau for Nuclear Measurements 
(CBNM), in Geel, Belgium, now known as the Institute for Reference Materials and Measurements (IRMM).

Because a CRM standard is considered a primary reference material, it must be available to a wide and perhaps international audience for an extended period of time. In light of these considerations, the designers of a CRM standard are severely limited in the quantity of SNM that can be put into that standard, mainly because of compliance concerns with the nuclear and other regulatory agencies in different laboratories in the US and in other countries where the CRM might be needed. These limitations are undoubtedly the reason why only two sets of CRMs for NDA have been produced in the past twenty years.

3. Working Reference Material. While WRMs should have quality characteristics similar to CRMs, the rigor used to achieve those characteristics is not usually as stringent as for CRMs. Where possible, CRMs are used to calibrate the methods used for establishing the concentration values or amount of SNM assigned to a WRM, thus providing traceability back to CRMs. A WRM is normally prepared for a specific NDA instrument at the facility where it is needed.

Because of the importance of having highly reliable measurement data from nuclear materials, particularly for control and accountability purposes, CRMs are sometimes used. However, CRMs prepared from SNM are not always available for specific applications. Thus there may be an urgent need for a laboratory or facility to prepare WRMs. The supply of CRMs is often too limited in the quantities needed for long-term, routine use. Well-characterized WRMs can appropriately be used in place of CRMs in most applications.

SNM solution standards are difficult to prepare and use because of the harsh chemical and physical properties. Chemical instabilities and radioactivity are two factors that must be dealt with carefully. Plutonium solution standards are always prepared locally at each site and only WRM solutions are available. It is difficult or impossible to ship solution standards from site to site because of compliance requirements from regulatory agencies. Thus in many cases, the only viable option is to prepare a WRM solution standard at the facility where it is needed.

Some WRMs are as well-characterized as the CRM standards. An example of this is the set of plutonium oxide and MOX standards at PERformance LAboratory (PERLA). ${ }^{2,3}$ However this is the only set fabricated and it is available for use only at PERLA.

\section{References}

1. D. Reilly, N. Ensslin, H. Smith, Jr., and S. Kreiner, eds., Passive Nondestructive Assay of Nuclear Materials (Office of Nuclear Regulatory Research, US Nuclear Regulatory Commission, Washington, DC, 1991), NUREG/CR-5550.

2. S. Guardini, G. Guzzi, F. Mousty, S. Baumann, E. Kuhn, and P. De Regge, "Quality Control Provisions Applied in the Preparation and Characterization of PuBearing PERLA Standards," Institute for Safety Technology, PERLA Safeguards Performance Laboratory. CEC report EUR-13038-EN (1990). 
3. S. Guardini, G. Guzzi, F. Mousty, C. Bigliocca, E. Kuhn, N. Doubek, R. Fiedler, A. Zoigner, S. Baumann, K. H. Nelges, G. Hesbacher, P. De Regge, L. Vandevelde, R. Boden, and D. Huys, "Pu PERLA Standards: Quality Control Provisions During Preparation and Analyses of the Samples," in Proc. 7th ESARDA Symposium on Safeguards and Nucl. Mater. Manage. (ESARDA, Ispra, Italy, 1985), ESARDA 19, p. 543. 


\section{OVERVIEW: GAMMA-RAY TECHNIQUES}

\section{A. Plutonium Isotopic Measurements}

1. Introduction. The role of standards in the nondestructive measurement of the isotopic composition of plutonium (and uranium) using gamma-ray spectroscopy is significantly different than for standards used with other NDA methods. These differences arise because the method most highly developed and used does not require calibration with standards, relying instead on known values of fundamental physical constants and internal or intrinsic self-calibration. This approach allows the measurement of the isotopic composition of the plutonium in samples of arbitrary size, shape, and physical and chemical composition inside containers of arbitrary and unknown size and composition, with a measurement geometry that is not required to be reproducible.

Nevertheless, standards still play an important role in the implementation and characterization of this measurement technique. It is only by using standards to check measurement performance that one can be assured that the measurement technique is indeed performing as required and that the accuracy or measurement bias can be characterized.

In the following sections we will develop the basis for these measurements that makes them independent of standards, discuss reference material standards that are available for measurement characterization, and describe how facilities may use working standards for the same purpose.

2. Measurement Principles. The photopeak area for a single gamma ray can be written as

$$
\mathrm{C}\left(\mathrm{E}^{\mathrm{i}}\right)=\lambda^{\mathrm{i}} \mathrm{N}{ }^{\mathrm{i}} \mathrm{BR}{ }_{\mathrm{j}}^{\mathrm{i}} \varepsilon(\mathrm{E}) \quad \text {, }
$$

where $C\left(E_{j}^{i}\right)=$ photopeak area of gamma-ray $j$ with energy $\mathrm{E}_{j}$ emitted from isotope $i$,

$\lambda^{i}=$ decay constant of isotope $i\left(\lambda^{i}=\ln 2 / T_{1 / 2}^{i}\right.$, where $T_{1 / 2}^{i}$ is the half-life of isotope $I$ ),

$\mathrm{N}^{i}=$ number of atoms of isotope $i$,

$B R_{j}^{i}=$ branching ratio (gamma rays/disintegration) of gamma-ray $j$ from isotope $I$, and

$\varepsilon\left(E_{j}\right)=$ total efficiency for photopeak detection of a gamma ray with energy $E_{j}$. Includes detector efficiency, geometry, sample selfabsorption, and attenuation in materials between the sample and detector.

When this equation is applied to gamma-ray $j$ from isotope $i$ and a second gammaray $m$ from a second isotope $k$ and rearranged, the atom ratio of the two isotopes $i$ and $k$ is given by 


$$
\frac{N^{i}}{N^{k}}=\frac{C\left(E_{j}^{i}\right)}{C\left(E_{m}^{k}\right)} \cdot \frac{T_{1 / 2}^{i}}{T_{1 / 2}^{k}} \cdot \frac{B R_{m}^{k}}{B R} \frac{R E\left(E_{m}^{i}\right)}{R E\left(E_{j}\right)}
$$

In Eq. 1 and 2, the photopeak areas $C(E)$ are measured and the half-lives $T_{1 / 2}$ and branching ratios $B R$ are known nuclear data. The total efficiency $\varepsilon(E)$ in Eq. 1 has been expressed in terms of the relative efficiency $R E$ in Eq. 2. Geometry factors cancel and the relative efficiency ratio includes only sample self-absorption, attenuation in materials between the sample and detector, and detector efficiency. The use of an efficiency ratio removes the need for reproducible geometry and makes the isotopic ratio method applicable to samples of arbitrary size, shape, and composition.

At this point the relative efficiency in Eq. 2 is still unknown, although it is only a function of energy. Rearranging Eq. 1 gives

$$
R E\left(E_{j}\right)=\left[\lambda^{i} N^{i}\right] \frac{C\left(E_{j}^{i}\right)}{B R_{j}^{i}}
$$

In Eq. 3, the term in brackets is a constant for a given isotope; the relative efficiency as a function of energy may be obtained from the variation of $C\left(E_{\mathrm{j}}^{\mathrm{i}}\right) / B R_{\mathrm{j}}^{\mathrm{i}}$ with energy for a series of gamma-rays $j$ from a single isotope $i$. This variation with energy is the same for all isotopes in the sample (assuming no isotopic heterogeneities) and "curves" for several isotopes may be normalized to each other to provide more data for the relative efficiency curve. The relative efficiency curve is unique to each detector-sample-measurement combination and therefore must be obtained directly from the measurement data on each individual sample. The curve cannot be predetermined; instead, it is intrinsic or "selfdetermined" for every measurement.

Once the relative efficiency has been determined via Eq. 3, all the terms in Eq. 2 are known, giving a measurement of the ratio of isotope $i$ to isotope $j$ that is independent of calibration and standards. When Eq. 2 is used to determine the ratio of each isotope to a common isotope, the isotope ratio data may be combined to produce absolute isotope fractions.

Typical relative efficiency curves shown in Figs. 1 and 2 illustrate that, as expected, the curves are sensitive to the characteristics of the sample and detector.

3. Use of Standards. Standards still play an important role in isotopic measurements even though a complete isotopic distribution may be obtained without calibration or the use of standards. Their primary use is to verify that a particular implementation of the general measurement principles discussed above gives results of sufficient accuracy for the application. 


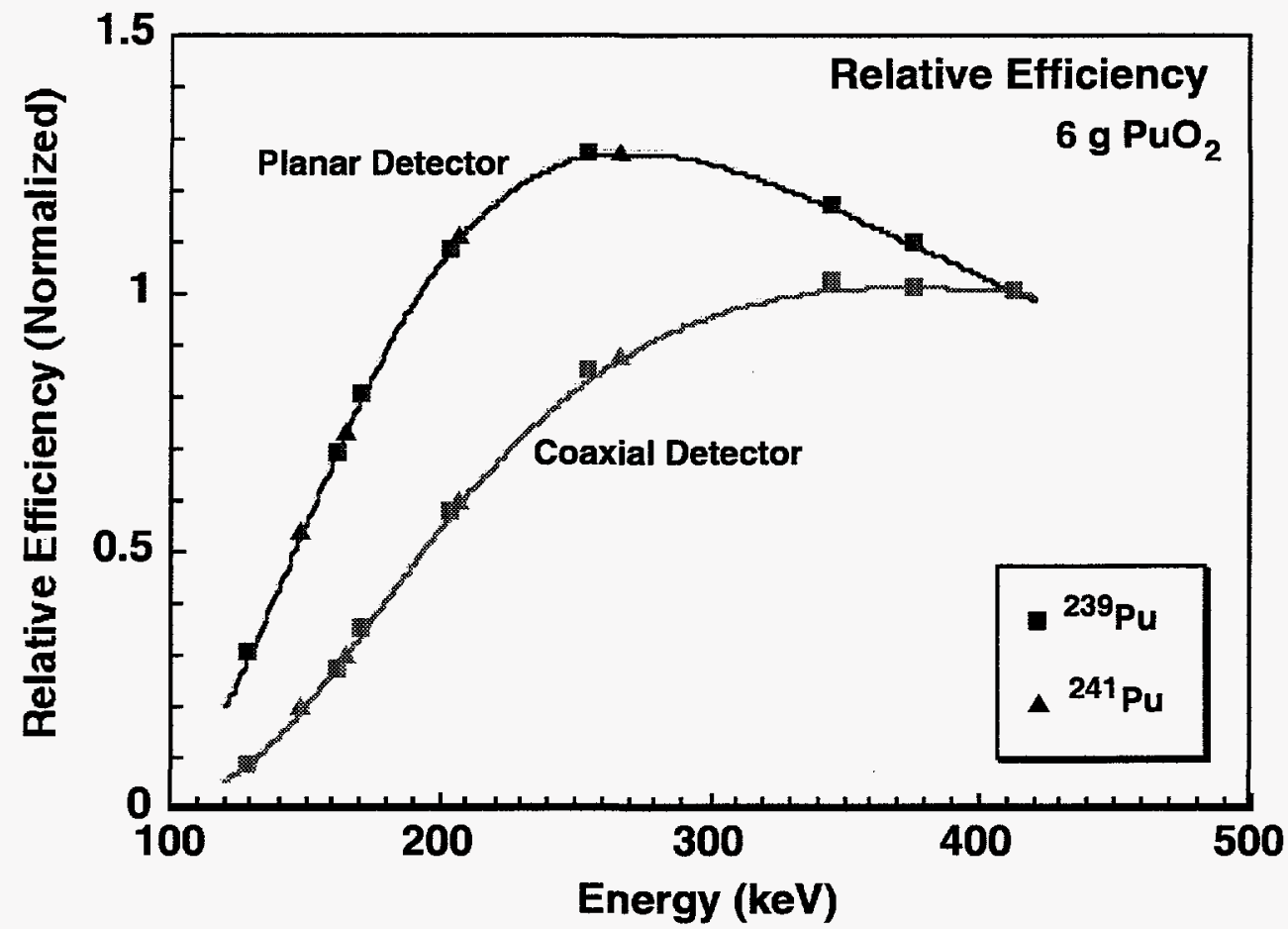

Fig. 1. Relative efficiency curves for a small plutonium sample measured with two types of detectors. The curves are constructed with data points from two isotopes.

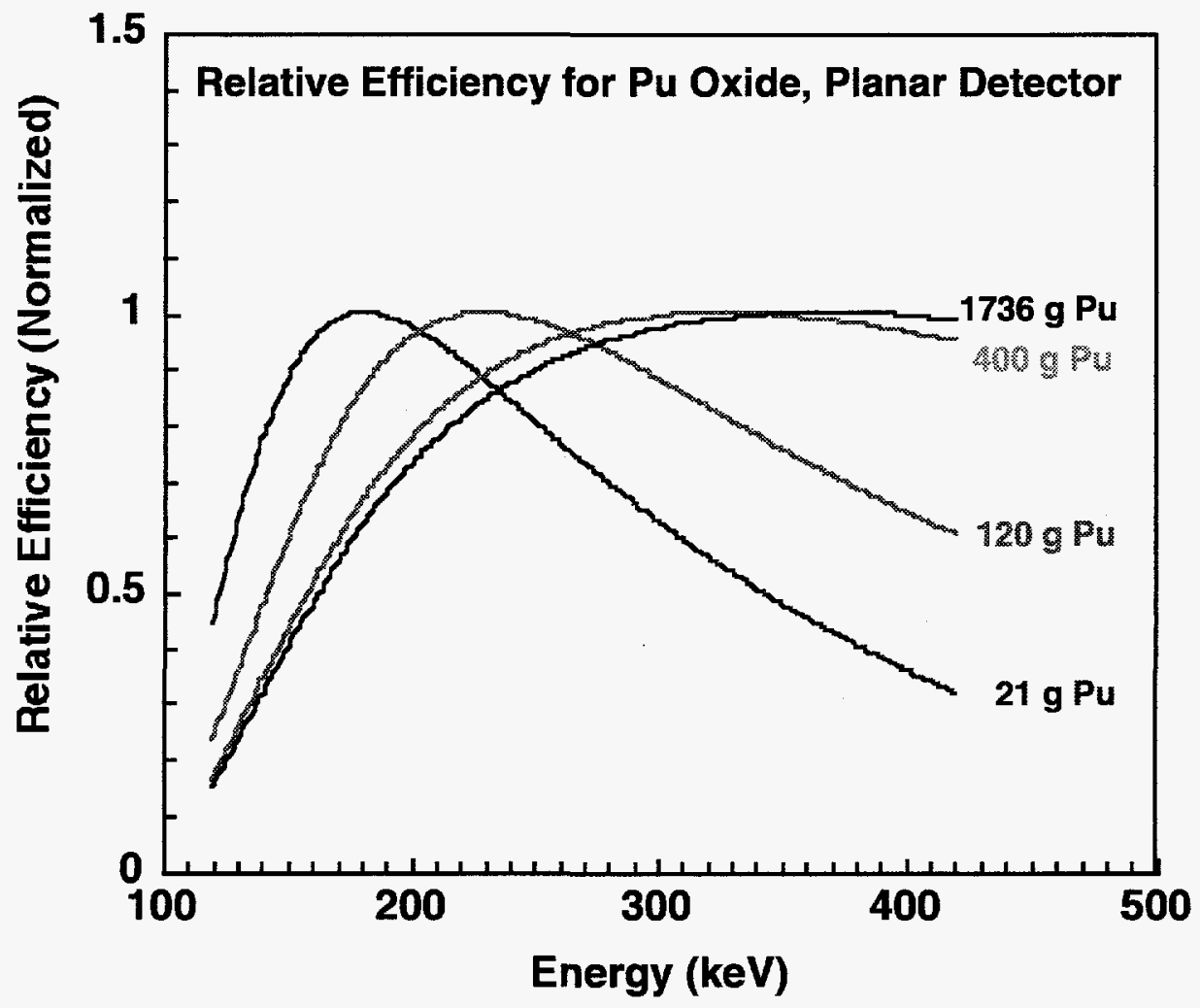

Fig. 2. Typical variation of relative efficiency showing effects of sample size when measured with the same detector. 
Using Eq. 2 in analysis algorithms to completely analyze a gamma-ray spectrum and produce a plutonium isotopic composition is a very complex process. Three plutonium isotopic analysis computer codes have been developed in the United States and are in use throughout the world. These codes are the $\mathrm{FRAM}^{2-4}$ code developed at Los Alamos, the MGA $^{5,6}$ code developed at Lawrence Livermore National Laboratory (LLNL), and the TRIFID $^{7}$ code developed at Rocky Flats. These codes differ somewhat in the energy ranges analyzed and in the detailed methods for obtaining peak areas and relative efficiency curves but share a common feature in that they include empirical correction factors to fine tune the results. The need for these factors arises because of imperfect peak-area analysis methods, imperfect modeling of the relative efficiency curve, and uncertainties in the published nuclear data (mainly in the branching ratios). While the analysis results from these codes are not free from bias, the bias on individual isotopic fractions may be $<1 \%$, relative, under good measurement conditions. An indication of the improvement that these empirical adjustments may produce is shown in Figs. 3 and 4.

The results in Figs. 3 and 4 are displayed as the ratio of the measured value to the certified or accepted value. The accepted value in nearly all cases comes from a mass spectrometry (MS) measurement and, in some instances, an alpha spectrometry

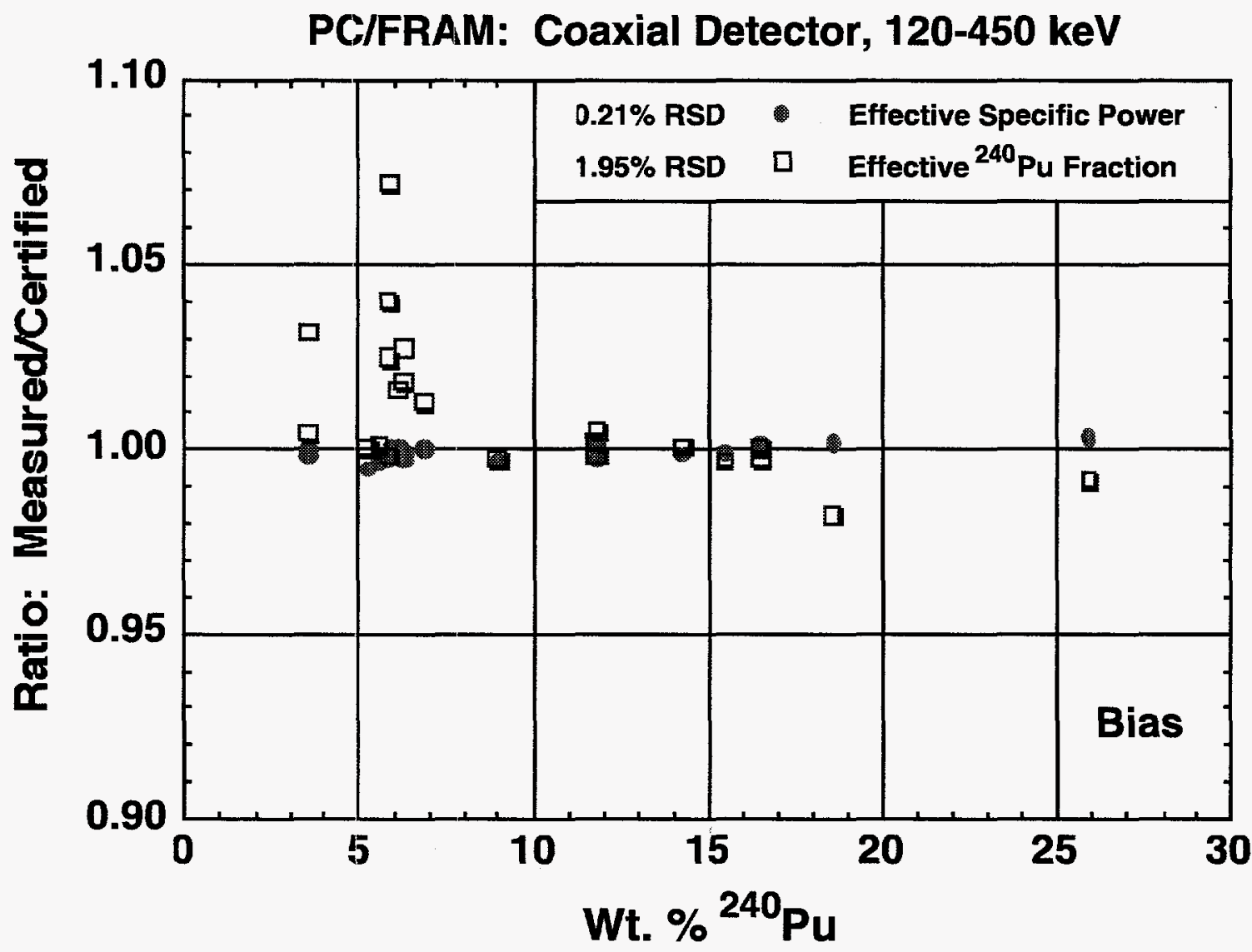

Fig. 3. The bias for two integral parameters calculated from the measured isotopic distribution. Values were calculated using an initial set of parameters. 


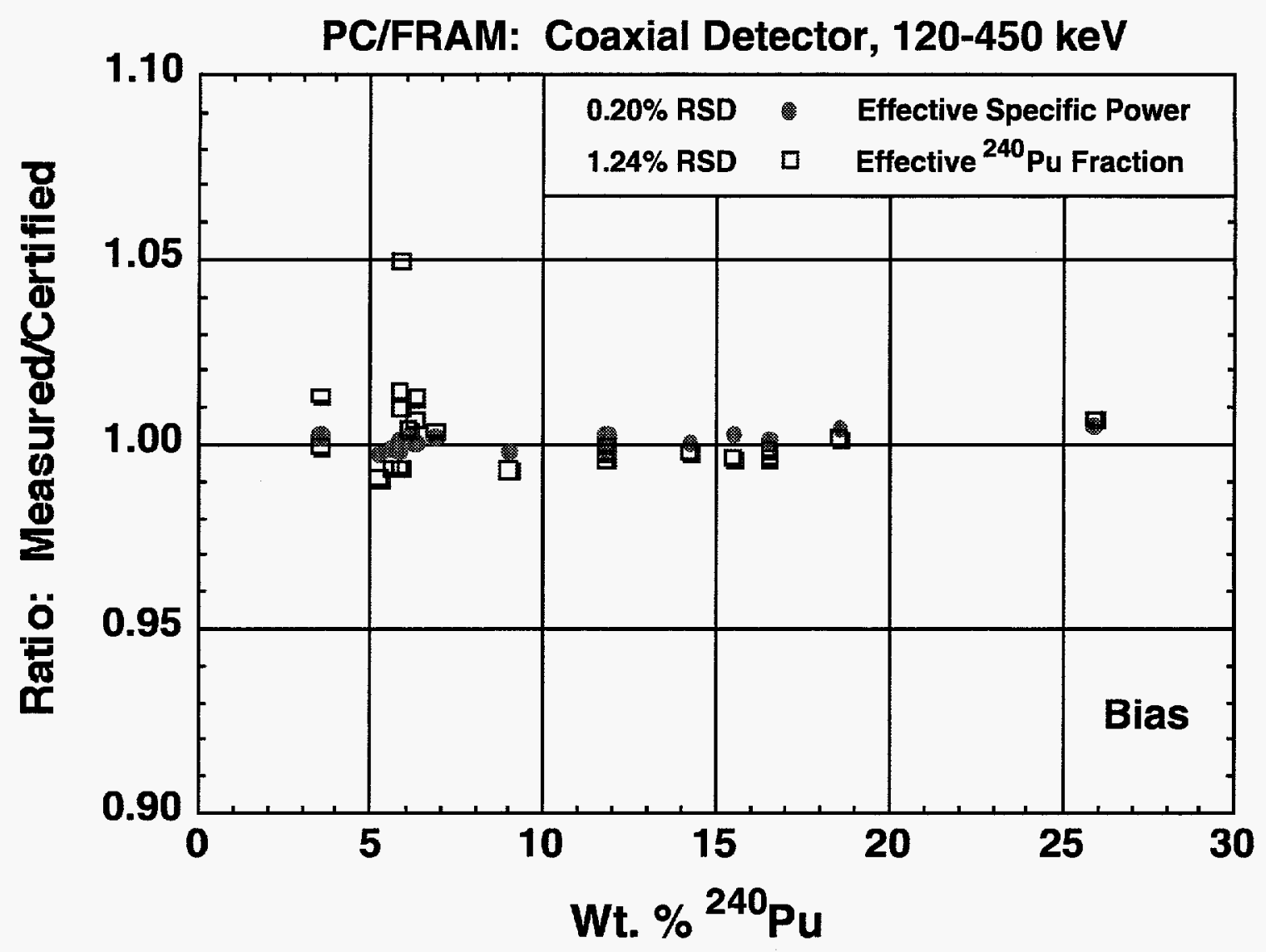

Fig. 4. Same data set as in Fig. 3 recalculated after parameter adjustments. The results for the effective ${ }^{240} \mathrm{Pu}$ fraction are significantly improved.

measurement for ${ }^{238} \mathrm{Pu}$. The existence of an MS value for the isotopic composition of an item is, for most purposes, all that is required to consider the item a "standard" for nondestructive measurements of the isotopic composition of plutonium.

We enclose the word "standard" in quotation marks to indicate that this may not be a standard in the true metrology sense, although it is very likely to be considered as one at some level in the standards hierarchy. In most cases, the accuracy and precision of the isotopic composition determined by MS is better than that for the nondestructive gammaray measurement, although there are exceptions for which the precision of the NDA measurement may exceed that of the destructive MS measurement. Most mass spectrometer measurements are carried out with strict quality control and with the use of CRMs for calibrating the mass spectrometer. The proper application of these longpracticed techniques in the analytical mass spectrometry laboratory will result in measurements that are traceable to the national measurement system and the resulting measured items may be considered as working standards in most instances. It is this widely accepted protocol that permits one to say that the isotopic measurements represented in Figs. 3 and 4 are being compared to "standards" when the item has an accepted value from MS carried out under controlled conditions with traceable MS standards. 
4. Examples of Standards. We present examples of standards in several categories. Standards can be used for direct comparison with nondestructive isotopic measurements and standards are also used to calibrate the mass spectrometers.

This second category of standards is important for NDA isotopic measurements because they are the conduit through which NDA working standards may be traceable to the national measurement system.

Both groups of standards can be represented by high-level standards, usually CRMs, and lower-level standards, usually called working standards.

a. Isotopic Reference Materials for NDA. Only two CRM standards have been prepared expressly for nondestructive isotopic analysis measurements.

CBNM Nuclear Reference Material 271 This standards set was prepared by the CBNM (now called the IRMM in Geel, Belgium, a laboratory of the Commission of the European Communities) and was issued with a certification date of 20 June 1986. The set consists of four sealed stainless steel containers each containing a sintered pellet of about $6.6 \mathrm{~g}$ of $\mathrm{PuO}_{2}$. The cans are $40 \mathrm{~mm}$ in diameter by $21 \mathrm{~mm}$ tall, each containing one of four isotopic compositions. They are labeled according to their nominal ${ }^{239} \mathrm{Pu}$ percentage as CBNM Pu 93, CBNM Pu 84, CBNM Pu 70, and CBNM $\mathrm{Pu} 61$. They are certified for ${ }^{238} \mathrm{Pu}^{242} \mathrm{Pu}$ and ${ }^{241} \mathrm{Am}$ isotopic fractions. Only a few sets of these standards exist in the United States (Los Alamos, LLNL, and NBL). The user should directly contact IRMM for availability.

EC Certified Nuclear Reference Material 171/NBL CRM 969 This set of lowenriched uranium standards issued in 1985 was prepared and certified jointly in the US and Europe. These standards were prepared primarily for uranium enrichment measurements but may also be used for nondestructive isotopic analysis measurements analogous to those developed for plutonium. The Los Alamos FRAM code can measure uranium in addition to plutonium while the MGAU code (a separate but similar code to MGA) was developed expressly for uranium. The set consists of five sealed aluminum alloy cans each containing $200 \mathrm{~g}$ of $\mathrm{U}_{3} \mathrm{O}_{8}$ in a different enrichment. The cans are $80 \mathrm{~mm}$ in diameter by $90 \mathrm{~mm}$ tall with nominal ${ }^{235} \mathrm{U}$ enrichments of $0.31 \%, 0.71 \%, 1.94 \%, 2.95 \%$, and $4.46 \%$. These materials may be obtained from the NBL in Argonne, IL.

b. Isotopic Reference Materials for Mass Spectrometry. Other standards are available for calibration of mass spectrometers. These standards provide the traceability to the national measurement system needed for NDA working standards whose pedigree arises from an isotopic analysis on a mass spectrometer calibrated with traceable materials.

CRM 136, 137, 138 (formerly National Bureau of Standards (NBS) 946, 947. 948) These reference materials are the primary US standards available for the calibration of mass spectrometers. Each standard contains about $0.25 \mathrm{~g}$ of plutonium as a sulfate. The nominal ${ }^{240} \mathrm{Pu}$ enrichments are $12 \%$ for CRM 136 (NBS 946), 18\% for CRM 137 (NBS 947), and 8\% for CRM 138 (NBS 948). 
These are large enough (barely) that they can also be used directly for nondestructive isotopic measurements. These standards are old; the original certification dates from 1972. Also, they are not certified for americium and the ${ }^{238} \mathrm{Pu}$ content of CRM 138 (NBS 948) is thought to have a significant bias. This CRM may be obtained from the NBL in Argonne, IL.

CRM 144 This reference material is a mixture of ${ }^{240} \mathrm{Pu}-{ }^{242} \mathrm{Pu}-{ }^{244} \mathrm{Pu}$ with accurately known fractions. It is used as a spike for isotope dilution mass spectrometry or as an internal standard to calculate the mass discrimination correction factor for mass spectrometers. This CRM may be obtained in 2-mg quantities from the NBL in Argonne, IL.

CRM 128 This reference material is an equal atom mixture of ${ }^{239} \mathrm{Pu}$ and ${ }^{242} \mathrm{Pu}$ intended primarily for the calibration of mass spectrometers. This CRM may be obtained in 1-mg quantities from the NBL in Argonne, $\mathrm{IL}$.

c. Working Standards for Isotopic Analysis. These lower-level standards are usually unique to a given facility and can be generally defined as materials for which the isotopic composition has been measured by an analytical laboratory operating under a qualified measurement control program. Many of the standards discussed elsewhere in this report, while fabricated specifically for other instruments, are also suitable for use as working standards for isotopic analysis. They can be used as working standards because an isotopic analysis is usually a part of any chemical characterization of a standard. This analysis is almost always sufficient to make a useful working standard for isotopic analysis.

Several types of standards appropriate for plutonium isotopic analysis are pictured in Fig. 5 below.

Counterclockwise from the upper right the following are pictured:

d. Segmented Gamma Scanner (tall stainless steel containers). These standards, consisting of well-characterized $\mathrm{PuO}_{2}$ mixed with diatomaceous earth, were fabricated for calibrating of segmented gamma-ray scanners (SGS). Because they have a very well known isotopic composition, they are also suitable for use as a working standard for isotopic analysis.

e. EC Certified Nuclear Reference Material 171/NBL CRM 969 (briefcase). These uranium enrichment standards are described above.

f. CRM 136, 137, 138, Formerly NBS 946, 947, 948 (contained in pipe nipples). These primary standards for mass spectrometry calibration may also be used for nondestructive gamma-ray isotopic analysis. The 0.25 -g sample is in a glass vial inside a cardboard container in the pipe nipple. 


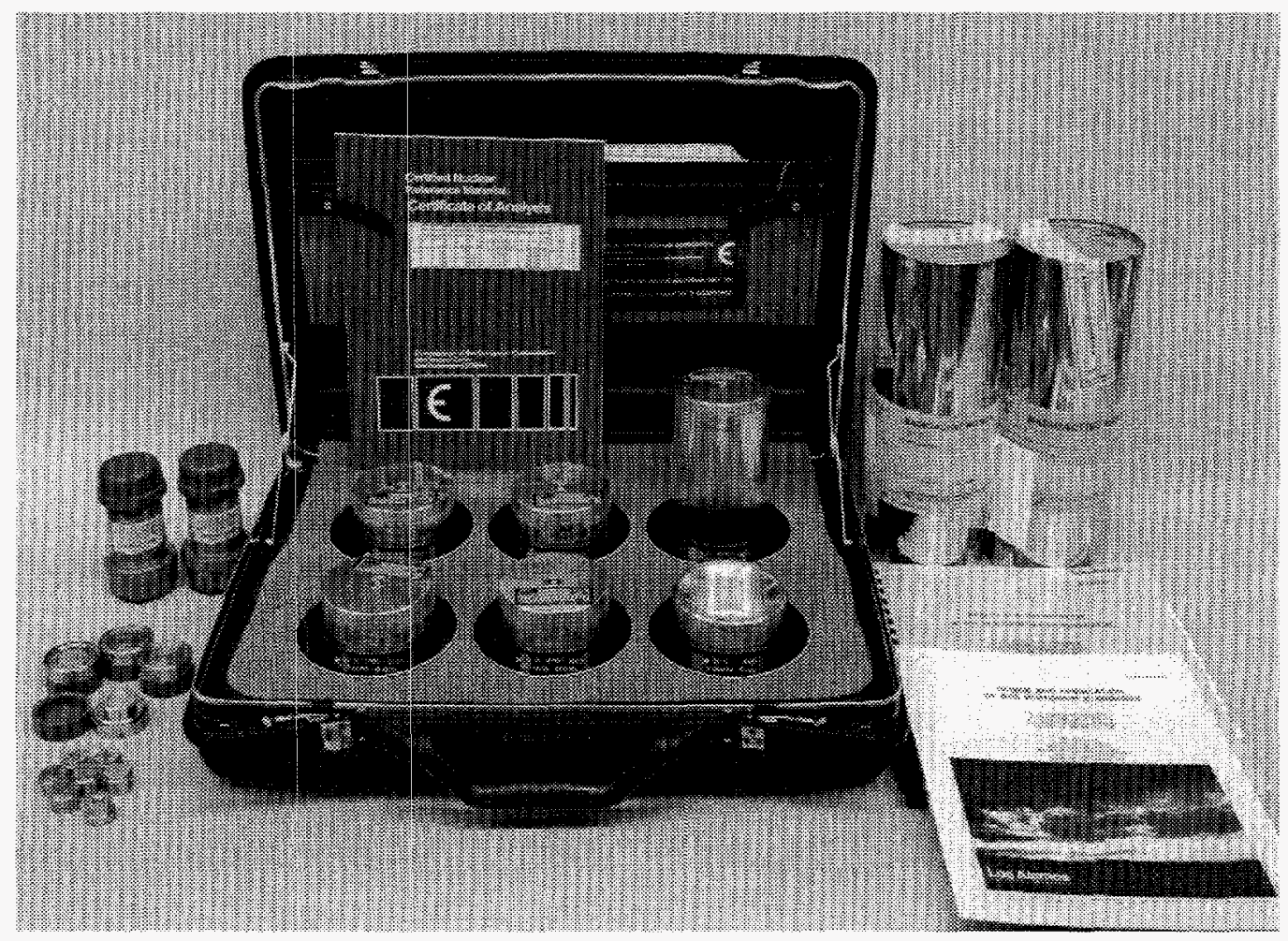

Fig. 5. Standards suitable for use in isotopic analysis by gamma-ray spectrometry.

g . CBNM Nuclear Reference Material 271. The set of four primary reference materials for nondestructive plutonium isotopic composition measurements (described above).

Plutonium Isotopic Determination Intercomparison Exercise (PIDIE) Standards A set of seven plutonium standards each containing about $0.4 \mathrm{~g}$ of plutonium with nominal ${ }^{240} \mathrm{Pu}$ isotopic percentages of $6.0,10.0,14.1,19.7,21.1,23.8$, and 25.5. These standards were distributed as unknowns for the exercise carried out in the late 1980s. While the values are not certified, they have been measured by several analytic laboratories and are reported in Ref. 8.

5. Calorimetry Exchange Program. The DOE supports a program to track the practice of calorimetry and plutonium isotopic composition measurements by gamma spectrometry throughout the complex. Seven laboratories participate in the program. Each laboratory regularly measures the isotopic composition and the total power from a standard $\mathrm{PuO}_{2}$ sample and reports the results, which are tabulated and distributed. The sample contains $400 \mathrm{~g}$ of plutonium as $\mathrm{PuO}_{2}$ with a nominal $6 \%{ }^{240} \mathrm{Pu}$ content. A group of identical standards was produced and characterized by Rocky Flats in 1979 from a single batch of plutonium. These standards were distributed to the participating laboratories and are still used for this purpose. The reference isotopic composition of this standard on 01 Jan 1994 is given in Table I. 
Table I

Reference Isotopic Composition of

Calorimetry Exchange Standard

01-Jan-1994

\begin{tabular}{|c|c|}
\hline Isotope & wt \% \\
\hline${ }^{238} \mathrm{Pu}$ & 0.0091 \\
${ }^{239} \mathrm{Pu}$ & 93.9172 \\
${ }^{240} \mathrm{Pu}$ & 5.8609 \\
${ }^{241} \mathrm{Pu}$ & 0.1837 \\
${ }^{242} \mathrm{Pu}$ & 0.0291 \\
${ }^{241} \mathrm{Am}$ & 0.1917 \\
\hline
\end{tabular}

Summary results for the calendar year 1994 are tabulated in Table II. These results represent the three isotopic analysis codes previously mentioned. The codes were operated according to facility-specific procedures.

Table II

Summary of Isotopic Composition Measurements of the DOE Calorimetry Exchange Standard in CY 1994

\% Difference from the Accepted Value

\begin{tabular}{|l|l|l|l|l|l|l|l|}
\hline & Livermore & $\begin{array}{l}\text { Los } \\
\text { Alamos }\end{array}$ & Mound & Hanford & $\begin{array}{l}\text { Rocky } \\
\text { Flats CSL }\end{array}$ & $\begin{array}{l}\text { Rocky } \\
\text { Flats AL }\end{array}$ & $\begin{array}{l}\text { Savannah } \\
\text { River }\end{array}$ \\
\hline$P_{\text {eff }}$ & -0.19 & 0.11 & 0.26 & 0.03 & no data & -2.09 & no data \\
$\%{ }^{238} \mathrm{Pu}$ & -1.56 & 4.74 & 6.70 & 2.21 & no data & 9.80 & no data \\
$\%^{239} \mathrm{Pu}$ & 0.02 & 0.00 & -0.02 & -0.05 & no data & -0.04 & no data \\
$\%^{240} \mathrm{Pu}$ & -0.25 & -0.14 & 0.83 & 0.83 & no data & 1.13 & no data \\
$\%^{241} \mathrm{Pu}$ & -0.66 & 0.38 & -0.50 & 0.17 & no data & 1.33 & no data \\
$\%^{241} \mathrm{Am}$ & -1.49 & 0.52 & 0.21 & -1.05 & no data & -0.85 & no data \\
\hline
\end{tabular}

6. Practical Considerations. Often when making comparisons between the measured and accepted values for isotopic composition measurements on standards (see Figs. 3 and 4 for example) we find the comparison not as good as desired. What is the cause of the problem? Three classes of problems may contribute to the disagreement.

- Problems with the analysis code

- Problems with the gamma-ray measurement process

- Problems with the "standard" or certified value 
The first class of problems has been discussed above and sometimes results in a solution involving fine-tuning of the analysis parameters. (We do not consider software bugs in this category.) It is all too easy to propose this as a solution in every case. However, when such fine-tuning begins to exceed the limits of reasonable physical principles, other solutions should be examined. Fine-tuning is not a solution to the other two categories and often the real problem involves determining the exact cause of the disagreement. The solution to problems with the analysis code may involve investigation of other codes which might have different areas of optimal application.

Problems with the gamma-ray measurement process usually involve gamma-ray spectroscopy practices. The old saying, "You can't make a silk purse out of a sow's ear," certainly applies when considering the source of disagreement between measured and standard values. You must have high-quality data to get high-quality results.

a . Improper Filtering. Too little filtering will result in intense low-energy gamma-ray peaks (especially ${ }^{241} \mathrm{Am}$ at $59.5 \mathrm{keV}$ ) that will pile up and sum with other peaks providing spectral distortion and interferences, which may cause biases.

b. Improper Count Rates. Count rates that are too high cause spectral distortions and pile up and summing peaks, which may bias the analysis. Count rates that are too low may result in peaks so weak that the analysis algorithms fail or the statistical precision is unacceptably poor.

c. Poor Quality Detector. The detector quality is the most important element of a good measurement system. Excessive exposure to neutrons will slowly degrade detector performance. The resulting increase in peak widths and degradation of peak shapes may bias the peak-area determinations in the analysis software.

d. Unanticipated Isotope Interferences. The analysis algorithms in plutonium isotopic analysis software may not adapt to interfering gamma-ray peaks that are not considered in the analysis.

The third class of problems involves errors or uncertainties in determining the "standard" or certified value. The values from the analytical laboratory must always be critically examined. The existence of a quality assurance program in the analytical laboratory is a necessary, but not a sufficient condition to guarantee quality standards. We have found that we must consider the following factors.

e. Source of Values for ${ }^{238} \mathrm{Pu}$. The ${ }^{238} \mathrm{Pu}$ isotopic fraction may be determined in the analytic laboratory by mass spectrometry or radiochemistry. One potential problem with mass spectrometry for ${ }^{238} \mathrm{Pu}$ is contamination of the small sample (often nanograms) with environmental ${ }^{238} \mathrm{U}$, which will register as ${ }^{238} \mathrm{Pu}$ and bias the measurement high. This may be a problem only for low-burnup plutonium with very low levels of ${ }^{238} \mathrm{Pu}$ (in the $0.01 \%$ range).

f. Am-241 Separation. In a similar fashion ${ }^{241} \mathrm{Am}$, which is always present in plutonium, will interfere with the determination of ${ }^{241} \mathrm{Pu}$. Procedures for preparing the sample for mass spectrometry of plutonium include a chemical separation step to remove 
the americium from the plutonium. If this separation is incomplete, the ${ }^{241} \mathrm{Pu}$ value may be biased high.

g. Units of Reported Results. This is a rather mundane question but is noted here because this author has been victimized by it. Americium-241 is usually reported by the analytical laboratory as a fraction of the sample analyzed. Sometimes the reporting unit is ppm (parts per million). If this is the only unit reported, the user must ask the question "Parts per million of WHAT?" If the sample was submitted as $\mathrm{PuO}_{2}$, is the result with respect to plutonium or $\mathrm{PuO}_{2}$ ? The difference is about $12 \%$. Nondestructive isotopic analysis measurements usually report ${ }^{241} \mathrm{Am}$ with respect to elemental plutonium.

\section{Conclusions}

Standards play an important role in the isotopic analysis of plutonium by gammaray spectrometry even though the basic technique is one of the few NDA techniques that does not require calibration with standards.

The primary use of standards with this technique is to provide reference values for comparison and testing against the NDA results, to validate the technique for MC\&A purposes, and to provide calibration materials for mass spectrometers for production of working standards for NDA.

\section{References}

1. D. Reilly, N. Ensslin, H. Smith, Jr., and S. Kreiner, eds., Passive Nondestructive Assay of Nuclear Materials (Office of Nuclear Regulatory Research, US Nuclear Regulatory Commission, Washington, DC, 1991), NUREG/CR-5550.

2. T. E. Sampson, G. W. Nelson, and T. A. Kelley, "FRAM: A Versatile Code for Analyzing the Isotopic Composition of Plutonium from Gamma-Ray Pulse Height Spectra," Los Alamos National Laboratory report LA-11720-MS (December 1989).

3. T. A. Kelley, T. E. Sampson, and D. DeLapp, "PC/FRAM: Algorithms for the Gamma-Ray Spectrometry Measurement of Plutonium Isotopic Composition," presented at the American Nuclear Society Fifth International Conference on Facility Operations-Safeguards Interface, Jackson Hole, Wyoming, September 2429, 1995. Los Alamos National Laboratory document LA-UR-95-3326 (September 1995).

4. T. E. Sampson, T. A. Kelley, T. L. Cremers, T. R. Konkel, and R. J. Friar, "PC/FRAM: New Capabilities for the Gamma-Ray Spectrometry Measurement of Plutonium Isotopic Composition," presented at the American Nuclear Society Fifth International Conference on Facility Operations-Safeguards Interface, Jackson Hole, Wyoming, September 24-29, 1995. Los Alamos National Laboratory document LA-UR-95-3287 (September 1995). 
5. R. Gunnink, "MGA: A Gamma-Ray Spectrum Analysis Code for Determining Plutonium Isotopic Abundances, Volume 1, Methods and Algorithms," Lawrence Livermore National Laboratory report UCRL-LR-103220, Vol 1 (April 1990).

6. R. Gunnink and W. D. Ruhter, "MGA: A Gamma-Ray Spectrum Analysis Code for Determining Plutonium Isotopic Abundances, Volume 2, A Guide to Using MGA," Lawrence Livermore National Laboratory report UCRL-LR-103220, Vol 2 (September 1990).

7. J. G. Fleissner, T. W. Coressel, D. A. Freier, L. L. Macklin, "TRIFID, A Second Generation Plutonium Isotopic Analysis System," Nucl. Mater. Manage. (Proc. Issue) XVIII, 814 (1989).

8. J. Morel and B. Chauvenet, "Intercomparaison des Mesures de Composition Isotopique du Plutonium Par Spectrometrie X Et Gamma Resultats de L'Action 'PIDIE,' Rapport Final,' Report CEA-R-5582, Centre D'Etudes Nucleaires de Saclay (December 1991). 


\section{B . Uranium Enrichment Measurements}

1. Measurement Principles. The radioactive ${ }^{235} \mathrm{U}$ isotope decays by alpha emission to excited levels of ${ }^{231} \mathrm{Th}$, which in turn emit gamma rays of various energies. The most prominent gamma line observed has the energy of $185.7 \mathrm{keV}$. If the uranium sample is large, gamma rays originating from ${ }^{235} \mathrm{U}$ atoms deep inside the sample are completely absorbed and do not contribute to the gamma radiation observed at the sample surface. Thus, with increasing sample thickness the $186-\mathrm{keV}$ gamma-ray flux at the surface reaches an equilibrium value, which is almost independent of the physical form of the sample. For pure uranium compounds this value is proportional to the ${ }^{235} \mathrm{U}$ enrichment of the sample: generally only small corrections for chemical composition have to be applied. This non-intuitive result is known as the "enrichment meter" principle. Its appropriate application requires that the sample be thick enough to be impenetrable for 186$\mathrm{keV}$ gamma radiation.

The gamma-spectrometric determination of the ${ }^{235} \mathrm{U}$ enrichment of bulk samples requires an exact measurement of the number of $186-\mathrm{keV}$ photons emitted from the sample per unit time for a fixed counting geometry. Therefore, all measurement parameters that affect the observed counting rate must be carefully controlled and corrected for. Crucial measurement parameters of this gamma-spectroscopic enrichment assay technique are shown schematically in Fig. 6.

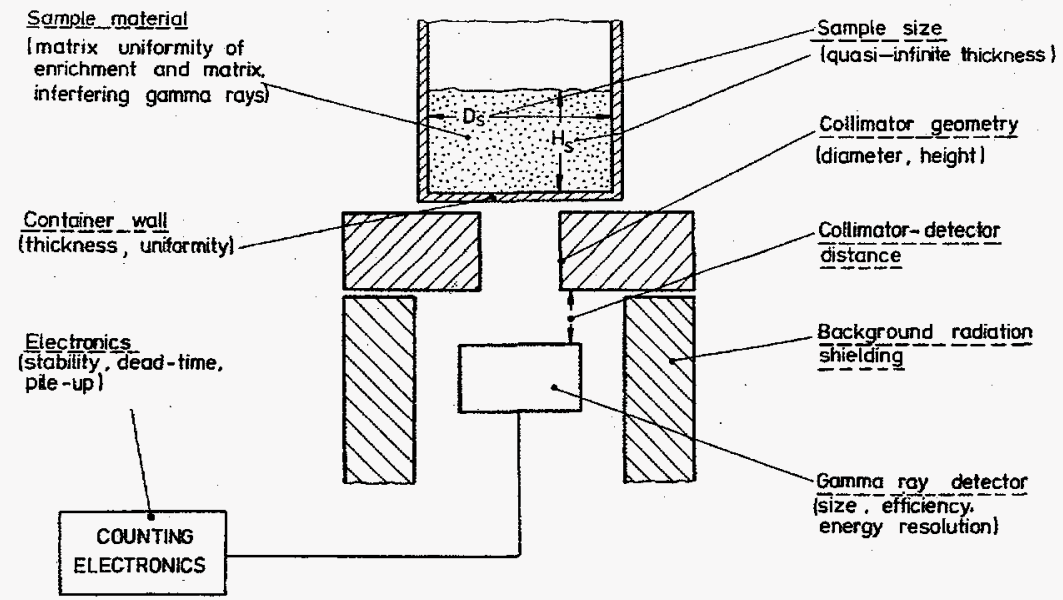

Fig. 6. Parameters affecting the accuracy of gamma-ray-spectroscopic ${ }^{235} \mathrm{U}$ enrichment measurements based on the "enrichment meter" principle.

The relation between the ${ }^{235} \mathrm{U}$ enrichment $E_{5}$ and the net peak counting rate $N_{186}$ of $186-\mathrm{keV}$ photons, observed with a gamma-ray detector is influenced by many factors, as shown in Eq. $4\left(E_{5}\right.$ is given in \%):

where

$$
E_{5}=100 \bullet \mathrm{N}_{186} \bullet \frac{4 \bullet \sigma \bullet T_{1 / 2}}{\ln 2 \cdot \mathrm{P}_{186}} \cdot \frac{1}{\mathrm{~F} \bullet \Omega \cdot \varepsilon} \cdot C_{\mathrm{Ma}} \cdot \mathrm{C}_{\mathrm{Wa}} \cdot \mathrm{C}_{\mathrm{El}} \bullet \mathrm{C}_{\mathrm{Int}},
$$


$N_{186}=$ observed net peak counting rate of $186-\mathrm{keV}$ photons,

$\sigma=$ photon attenuation cross section at $186 \mathrm{keV}$ for uranium,

$T_{1 / 2}=$ half-life of ${ }^{235} \mathrm{U}$,

$P_{186}=$ branching ratio of $186-\mathrm{keV}$ photons,

$F=$ collimator cross section,

$\Omega=$ solid angle formed by collimator and detector,

$\varepsilon \quad=$ intrinsic detector efficiency,

${ }^{\mathrm{c}} \mathrm{Ma}=$ correction for gamma attenuation in sample matrix materials,

${ }^{\mathrm{C}} \mathrm{Wa}=$ correction for gamma attenuation in sample container wall,

${ }^{c} E l=$ correction for counting rate losses caused by counting electronics, and

${ }^{c}$ Int $=$ correction for interference due to photons from isotopes other than ${ }^{235} \mathrm{U}$.

A detailed discussion of this equation can be found in Ref. 1.

2. Physical Criteria for Standards. An enrichment standard has the following requirements:

- The sample must be "quasi-infinitely" thick for $186-\mathrm{keV}$ gamma rays. This condition inherently restricts the method to relatively thick samples. This is discussed below.

- The sample must be uniform with respect to ${ }^{235} \mathrm{U}$ enrichment. When mixtures of differently enriched materials are assayed, the sample material must be carefully homogenized prior to the measurement.

- The container wall thickness must be reasonably thin so that the correction for attenuation in the wall is relatively small. If the container wall is thick, then the gamma attenulation correction must be experimentally determined.

3. The "quasi-infinite" Sample Size. Enrichment measurements using the "enrichment meter" principle will give accurate results only if the sample is sufficiently thick to be opaque for $186-\mathrm{keV}$ gamma rays. For a uniform sample the minimum thickness required for $99.9 \%$ of the infinite-thickness response is given in Table III. (valid for extremely narrow collimators).

The actual thickness of sample needed is less than that shown in Table III. This is explained as follows. In an actual measurement, a collimator of finite geometry would be used to achieve adequate results in a reasonable counting time. The transmission of photons (through the sample) that are viewed by the detector is described by the mean-freepath length through the sample. Because the mean-free-path length is actually an average over many directions and only one direction (through the sample) is applicable in a detector-collimator measurement, it follows that the sample thickness required is smaller than the linear sample thickness as given in Table III. For most collimator-detector arrangements, an estimate of the "quasi-infinite" sample dimension can be defined and calculated. ${ }^{1}$ This is represented graphically in Fig. 7. 
Table III

Linear Sample Dimension Required for $99.9 \%$ Gamma Response

\begin{tabular}{|l|c|c|c|}
\hline \multicolumn{1}{|c|}{ Uranium Compound } & Mass Attenuation & Sample Density & Sample Thickness \\
\hline & coef. $\left(\mathrm{cm}^{2} \cdot \mathrm{g}^{-1}\right)$ & $\left(\mathrm{g} . \mathrm{cm}^{-3}\right)$ & $(\mathrm{cm})$ \\
\hline $\mathrm{U}$ metal & 1.47 & 19.0 & 0.25 \\
\hline $\mathrm{UO}_{2}$ & 1.31 & 1.0 & 5.27 \\
\hline & & 2.0 & 2.64 \\
\hline & & 11.0 & 0.48 \\
\hline $\mathrm{U}_{3} \mathrm{O}_{8}$ & 1.27 & 1.0 & 5.44 \\
\hline & & 2.0 & 2.72 \\
\hline & & 8.3 & 0.66 \\
\hline $\mathrm{UF}_{6}$ & 1.03 & 1.0 & 6.71 \\
\hline & & 4.7 & 1.43 \\
\hline Uranyl nitrate & 0.77 & 1.0 & 8.97 \\
\hline & & 2.8 & 3.20 \\
\hline
\end{tabular}

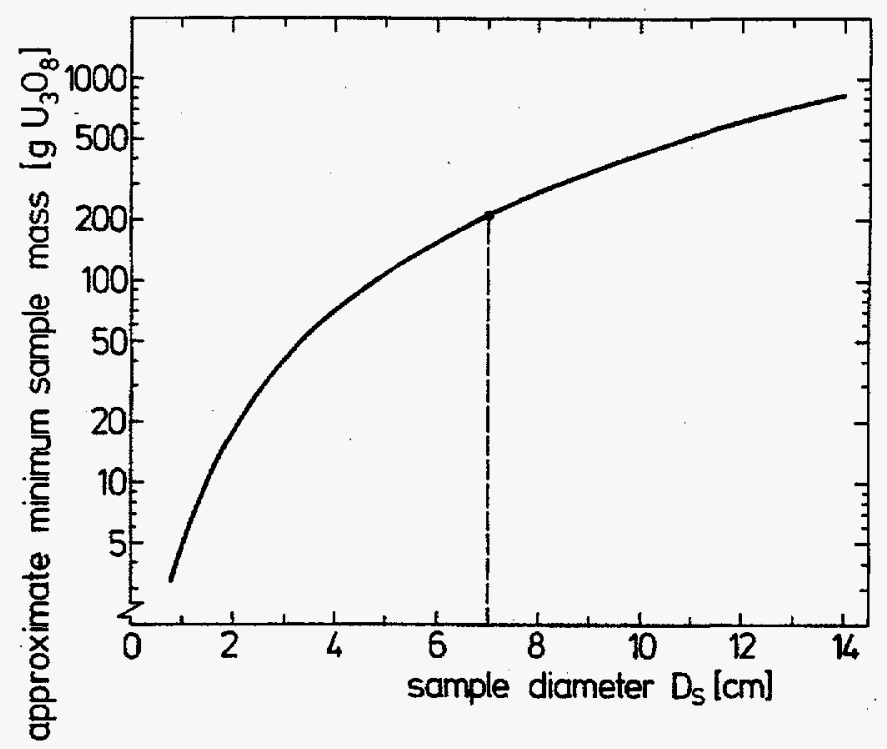

Fig. 7. "Approximate-minimum" $\mathrm{U}_{3} \mathrm{O}_{8}$ sample mass required for $99.9 \%$ of the infinite response for a cylindrical sample as a function of the sample diameter.

For 7-cm-diameter samples, the approximate minimum values of the sample mass for $\mathrm{UO}_{2}$ and $\mathrm{U}_{3} \mathrm{O}_{8}$ powders are

$$
\begin{array}{ll}
M_{s}=202.5 \mathrm{~g} & \text { for } \mathrm{UO}_{2} \text { powder and } \\
M_{s}=209.7 \mathrm{~g} & \text { for } \mathrm{U}_{3} \mathrm{O}_{8} \text { powder. }
\end{array}
$$

The CRM EC-NRM-171/NBL-SRM-969 is based on this calculation with $200 \mathrm{~g}$ of $\mathrm{U}_{3} \mathrm{O}_{8}$ in each standard, with a sample height of $2 \mathrm{~cm}$. This is to minimize the amount of SNM required. 
4. Preparation and Examples of Standards. Two sets of enrichment standards should be mentioned: one is the EC-NRM-171/NBS-SRM-969, which was jointly certified by the CBNM* in Geel, Belgium, and by the US NBS. ${ }^{*}$ This set is very well documented. ${ }^{1}$ The only unfortunate aspect is that the enrichment ranges from $0.3 \%$ to $4.5 \%$, which is adequate only for the low-enrichment fuel cycle. We have been told that IRMM and NBL are preparing additional standards with higher enrichments. Details of this CRM are discussed in Chapter IV and will not be repeated here.

The second is a of WRMs at Los Alamos. This set has $191-\mathrm{kg}$ cans of uranium oxide with 12 enrichments between normal and 91 at.\% that were acquired over a period of many years. They were packaged in various containers; isotopic ratios and uranium fractions were only routinely determined and based on a single sample. In 1985, the Safeguards Assay group repackaged and recharacterized these 19 uranium oxide samples. ${ }^{2}$

Of the 19 WRMs, seven were found to be homogeneous and can be called primary enrichment standards. Even though the oxides were blended previously, some were found to be isotopically inhomogeneous. To assure sample homogeneity, dissolving the oxide in solution and precipitation, as was done with the CRM EC-NRM-171/NBS-SRM-969, should be part of the fabrication procedure. We will describe the sampling and characterization of these standards.

From each of the 19-kg lots of uranium oxide, six 2-g samples were collected from different parts of the oxide. Of the six samples, three were analyzed by Los Alamos, two were submitted to the DOE's NBL for similar analyses, and one sample was put into archival storage for possible future analyses. The most important analysis is of course the isotopic distribution; the uranium fraction is of secondary importance. The isotopic distributions and other important parameters of the seven primary standards are shown in Table IV.

Table IV

Parameters of Primary Enrichment Standards at Los Alamos

\begin{tabular}{|c|c|c|c|c|c|}
\hline Standard ID & ${ }^{235} \mathrm{U}$ & Std. Dev. & U mass & Approx. Oxide & Approx. Oxide \\
\hline & $($ at.\%) & \pm & $(\mathrm{g})$ & Depth $(\mathrm{cm})$ & Density $\left(\mathrm{g} / \mathrm{cm}^{3}\right)$ \\
\hline UISO-91 & 91.419 & 0.011 & 990 & 7.7 & 1.7 \\
\hline UISO-66 & 66.317 & 0.032 & 990 & 5.6 & 2.3 \\
\hline UISO-52 & 52.426 & 0.004 & 989 & 5.8 & 2.2 \\
\hline UISO-38 & 37.848 & 0.015 & 991 & 6.0 & 2.1 \\
\hline UISO-13 & 13.098 & 0.008 & 991 & 5.4 & 2.4 \\
\hline A1-324-1 & 10.202 & 0.003 & 987 & 4.6 & 2.8 \\
\hline A1-324-2 & 10.200 & 0.001 & 987 & 4.1 & 3.1 \\
\hline
\end{tabular}

\footnotetext{
* The Central Bureau of Nuclear Measurements (CBNM) in Geel, Belgium, has changed its name to the Institute of Reference Materials and Measurements (IRMM). The US National Bureau of Standards (NBS) has changed its name to the National Institute of Standards and Technology (NIST). The agent that sells the CRM standards for NIST is the New Brunswick Laboratory (NBL) at Argonne, Mllinois.
} 
These seven uranium oxide standards span the range from $91 \%$ to $10 \%$ and complement very well the CRM EC-NRM-171/NBS-SRM-969 set. To perform enrichment measurements over a wide enrichment range, both sets of standards should be used.

\section{References}

1. P. Matussak, "Accurate Determination of the ${ }^{235} \mathrm{U}$ Isotope Abundance by Gamma Spectrometry: A User's Manual for the Certified Reference Material ECNRM-171/NBS-SRM-969," Institut für Kernphysik report KfK 3752, Kernforschungszentrum, Karlsruhe, Federal Republic of Germany (1985).

2. J. L. Parker and M. Brooks, "Accurate, Wide-Range Uranium Enrichment Measurements by Gamma-Ray Spectroscopy: I. Characterization of Enrichment Standards; II. Demonstration of Enrichment Measurement Capability," Los Alamos National Laboratory report LA-11277-MS (September 1988). 


\section{Solutions}

1. Measurement Principles. Solutions containing SNM are ideal samples for NDA because they are usually uniform and can be tailored to the assay technique by the selection of appropriate vials. There are three different methods of assaying solution samples:

- transmission-corrected passive counting of ${ }^{235} \mathrm{U}$ and ${ }^{239} \mathrm{Pu}$ gamma-ray peaks using the solution assay instrument (SAI) in near-field geometry,

- $\quad x$-ray fluorescence (XRF), and

- the K-edge absorption densitometry (KED) technique. Details of these techniques can be found in Passive Nondestructive Assay of Nuclear Materials. $^{1}$

The SAI typically includes an up-looking detector viewing the entire solution sample contained in cylindrical sample vials. ${ }^{1}$ Because solutions are usually uniform, the attenuation correction in the solution can be characterized by a linear attenuation factor. The attenuation factor can be determined by measuring the transmission of an external source. The transmission source may emit gamma rays with the same energy as that of the assay peak, or the source may have gamma rays with different energies. In the latter case, the transmission at energies near the assay peak can be extrapolated or interpolated to the assay peak energy. If this is done correctly, attenuation corrections accurate to $0.1 \%$ to $0.2 \%$ can be achieved over a wide concentration range. Rate-loss corrections, due to varying counting rates, are determined by monitoring a source $\left({ }^{109} \mathrm{Cd}\right)$ fixed to the detector. The unique aspect of the SAI technique is that because the detector is viewing the entire solution, one can actually determine the mass of ${ }^{235} \mathrm{U}$ and ${ }^{239} \mathrm{Pu}$ in the sample, not just the concentration. By combining the SAI measurement and the weight of the sample, one can determine the mass of ${ }^{235} \mathrm{U}$ and ${ }^{239} \mathrm{Pu}$ per gram of sample. Because the SAI technique involves monitoring the counting rate of the passive signals from ${ }^{235} \mathrm{U}$ and ${ }^{239} \mathrm{Pu}$ and it constitutes an absolute measurement, the calibration factor should be checked approximately every six months with standards.

The XRF technique is substantially different from the SAI technique. Instead of counting the passive gamma rays from uranium and plutonium, the $x$-rays induced in the sample by an external excitation source are measured. ${ }^{1}$ The excitation source can come from either a preselected radionuclide or a continuous-energy $x$-ray generator. Transmission corrections for the XRF technique are more complicated because of the excitation and subsequent de-excitation by $\mathrm{x}$-ray emission. First, one must determine the attenuation of the emission from the excitation source (gamma ray or $\mathrm{x}$-ray) interrogating the solution. Second, one must also determine the attenuation of the $x$-rays emerging from the solution, which are lower in energy. The problem of the attenuation at the two energies is further complicated by the fact that they are above and below the absorption edge of the element of interest, so simple interpolation of transmission does not provide the correct result. Rate-loss corrections required because of variable count rates are measured by monitoring a rate-loss source mounted close to the detector. The XRF technique constitutes an absolute measurement of the elemental uranium and plutonium concentrations 
in the sample. Therefore, the calibration should be checked every several months with WRM solution standards.

$\mathrm{KED}$ is unique in that it enables one to measure the transmission through a solution both above and below the KED of the element of interest. ${ }^{1}$ One can then determine the concentrations of the element from the ratio of the measured transmissions. The transmission source can either be a radionuclide selected for its gamma-ray emission energy, or it can be a continuous-energy $\mathrm{x}$-ray generator. Most KED analyses performed in nuclear facilities use an x-ray generator as the excitation source. X-ray-generator based KED has several distinct advantages; one advantage is that no rate loss correction is necessary because transmissions above and below the absorption edge are measured concurrently. These two transmissions can be used to determine uranium and plutonium concentrations simultaneously without chemical separation (within a certain concentration range). The typical KED detector is a side-looking detector measuring the transmission through a solution sample contained in a rectangular, optical absorption vial. In contrast to $\mathrm{SAI}$ and XRF, KED involves a measurement of the ratio of the transmissions through a solution over a narrow energy range, typically a few $\mathrm{keV}$ apart. Therefore the calibration factor should be relatively stable. We have evidence that the same calibration factor can be valid for up to several years.

2. Preparation of Standards. Solution WRMs are relatively easy to prepare, in principle, however, there are several practical considerations.

First, solution standards have a relatively short shelf life, for several reasons. Plutonium solutions have relatively high radiation-emission rates and therefore radiolysis rapidly becomes a significant problem, especially at higher concentrations. Because of the radiolysis, plutonium solution samples cannot be sealed tightly. The shelf life of a plutonium solution standard, i.e., the stability of the measured plutonium concentration, depends on the burnup characteristics of the plutonium. Typically one cannot trust the plutonium concentration after its determination if the solution is more than two weeks old. Uranium solutions have relatively low radiation emission rates and therefore have a longer shelf life; the shelf life of uranium solutions depends critically on the container. If the container is plastic, then the low- $\mathrm{Z}$ part of the matrix of the WRM (typically nitric acid) tends to diffuse out of the vial, thereby increasing the uranium concentration. This is not serious for the SAI technique, in which one is measuring the total ${ }^{235} \mathrm{U}$ and ${ }^{239} \mathrm{Pu}$ in the sample; a concentration change only affects the calibration in the correction factor, which is a second order effect. However, concentration changes directly affect the calibrations of both the XRF and KED techniques. If the uranium WRM is contained in a glass ampoule that is flame sealed, the solution concentration is stable for an extended period of time (months to years). If the vial is glass with a glass stopper, then it is critical that the vial is properly sealed. The sealant material for the glass vials must be investigated for its ability to prevent evaporation and for its resistance to nitric acid.

All three NDA techniques are capable of measurements at $0.2 \%$ precision if sufficient attention is devoted to the details of the measurement, the calculation of the correction factors, and the preparation of standards. Let us consider the preparation of a set of uranium standards with concentrations varying from low to high. By using the factor discussed in Chapter $\mathrm{V}$ of this report, the uncertainty of the standards should be a factor of 
5 lower than the required precision, or $0.05 \%$. The preparation of accurate WRMs at this level of precision is approaching the limits of standard chemical methods.

There are two methods of preparing a set of solution WRMs with concentrations varying from low to high. The first method is to prepare a master solution of the highest concentration and then determine the concentration of the master solution by means of standard chemical analysis techniques; the lower concentration standards can then be prepared by diluting the master solution. The second method is to prepare each WRM directly and characterize the concentration of each WRM independently by DA techniques. Both methods have their strengths and weaknesses. It is easier to prepare a set of solution WRMs that are internally consistent with each other by the first method, but the bias of the whole set of the lower-concentration (diluted) WRM standards depends heavily on the characterization of the master solution. WRM standards prepared by the second method have potentially more random error, but they are not subject to the bias of the characterization of one solution.

3. Dilution. To prepare standards of lower concentration from a master solution, one can use dilution on either a weight (mass) basis or a volumetric basis. The following example provides details of a typical dilution method that is based on weight measurements: a technique that, in general, is more forgiving than volumetric dilution.

a. Weigh the mixing container (MC)

b. For the desired nominal standard concentration, deliver

$w_{0}$ the required volume $V_{m}$ of master solution to $\mathrm{MC}$

c. Weigh $\mathrm{MC}$ and master solution

d. Deliver dilutant to $\mathrm{MC}$ to give the required volume, $V_{m}$, of mixed solution.

e. Weigh $\mathrm{MC}+$ master solution + dilutant

f. Cap and seal MC and mix well

g. Weigh empty standard vial (SV)

h. Deliver contents of MC, as completely as possible, to SV

i. Weigh SV + standard solution

j. Weigh $\mathrm{MC}+$ residual solution

$w_{1}$
$w_{2}$
$w_{3}$
$w_{4}$
$w_{5}$

Assume that the uranium concentration of the master solution is $r_{M}=\mathrm{g} \mathrm{U} / \mathrm{g}$ solution. This quantity has to be determined by acceptable chemical methods.

Volume of standard $=V_{s}=V_{m} *\left(1-\left(w_{5}-w_{0}\right) /\left(w_{2}-w_{0}\right)\right)$

Concentration of the sample $=R_{s}=\left(w_{1}-w_{0}\right) * r_{M} / V_{m}$

Grams of uranium per $\mathrm{g}$ of sample $=r_{s}=\left(w_{1}-w_{0}\right) /\left(w_{2}-w_{0}\right) * r_{M}$

The traceability of the WRM is the uranium concentration of the master solution, $r_{M}$, which should be determined by accepted chemical methods. This important chemical analysis should be verified by assaying appropriate CRM standards provided by NBL or 
CBNM. The balance used for weight (mass) determinations should be verified with NISTtraceable weights. Notice that the determination of the uranium concentration of the entire set of standards depends heavily on one chemical determination, namely the uranium concentration of the master solution, $r_{M}$. If this chemical analysis has a bias, then the entire set of solutions has the same bias. However, it is easier to produce an internally consistent set of WRM solution standards by this method.

Dilutions from a master solution of approximately one order of magnitude are acceptable by this method. For example, starting with a 300-g U/1 master solution, one can prepare a 30-g U// standard, or even a 10-g U/1 standard. To prepare WRM standards with uranium concentrations less than $10 \mathrm{~g} \mathrm{U} / 1$, one should prepare another master solution with a lower uranium concentration and properly characterize it. The main reason for this is to avoid the potentially higher $(\sim 0.5 \%)$ error in weighing a small volume (a few $\mathrm{mL}$ ) of master solution in the preparation of diluted solutions.

4. General Comments. All three NDA techniques are capable of producing assays at a precision of $0.2 \%$ if sufficient attention is devoted to the details of the measurement and the calculation of the correction factors. In fact, NDA methods can be used to check the consistency of a set of standards in a verification measurement. The KED method, because of the long-term stability of calibration standards, can readily be used to characterize uranium or plutonium solutions for use as WRM standards, thus saving considerable time and effort.

\section{Reference}

1. D. Reilly, N. Ensslin, H. Smith, Jr., and S. Kreiner, eds., Passive Nondestructive Assay of Nuclear Materials (Office of Nuclear Regulatory Research, US Nuclear Regulatory Commission, Washington, DC, 1991), NUREG/CR-5550. 


\section{Segmented Gamma Scanning}

1. Introduction. SGS is an NDA technique designed ${ }^{1}$ to use gamma-ray assay to quantitatively determine SNM in low-density scrap and waste samples. It is based on the observation that the SNM waste in facilities is usually layered in containers wherein the horizontal inhomogeneity is less pronounced than the vertical inhomogeneity. The vertical inhomogeneity effect can be overcome by assaying the sample in segments by means of a collimated gamma ray detector. The effect of horizontal inhomogeneity can be minimized by rotation of the sample during the assay.

Gamma-ray assay can be described by the following expression:

$$
T C R=R R * C F(R L) * C F(A T),
$$

where $T C R=$ total corrected rate,

$R R \quad=$ raw rate of data acquisition,

$C F(R L)=$ correction factor for rate-related electronic losses, and

$C F(A T)=$ correction factor for self-attenuation in sample.

If the correction factors are properly defined and computed, TCR is the data acquisition rate that would have been observed if there were no electronic losses and if the sample were changed to a point or line source with the same gamma-ray emission rate but no self-attenuation. TCR is proportional to the mass of the isotope emitting the gamma ray of interest:

$$
T C R=K * M,
$$

where $M$ is the mass of the isotope being assayed and $K$ is the calibration constant. The calibration constant $K$ is determined by the use of appropriate standards and it includes the effects of detector efficiency, subtended solid angle, and the gamma-ray emission rate. In SGS, each segment is measured individually and after all segments are measured the TCRs for all segments are summed as follows:

$$
T C R_{i}=R R_{i} * C F_{i}(R L) * C F_{i}(A T)
$$

The basic assumption of the SGS method is that the SNM is uniformly distributed within each segment and that the attenuation of the entire segment can be characterized by a single experimental transmission measurement because the attenuation factor $C F_{i}(A T)$ can be described mathematically ${ }^{2}$ in terms of a transmission. These mathematical calculations of $C F_{i}(A T)$ are valid only if the horizontal inhomogeneity is relatively small.

The following is a discussion of the requirements of SGS standards. The calibration factor should be known better than the precision and bias of the instrument by a 
factor of 2 to 5; at this level the contribution of the uncertainty in the calibration factor to the overall assay precision and bias will be relatively small. In general, the precision of SGS assays is $\sim 1 \%$ and the bias is $\sim 10 \%$ for low-density waste. Therefore the calibration constant for SGS should be known with a precision of $<0.5 \%$ and a bias of $<2 \%$. In general, the requirement for physical and chemical similarity between standards and unknowns is not important. However, several factors affect the measurement and are important for preparation of a standard:

- Uniformity of standard material,

- Transmission range,

- End effect considerations, and

- Particle size.

2. Uniformity. The ideal SGS standard would be a line source that has a range of source strengths with no self-attenuation, but nature did not provide such a standard. The best one can prepare is a standard with a uniform distribution of SNM. Uniformity is important for the calculation of the self-attenuation correction factor $C F(A T){ }^{1}$ Reference 1 gives a detailed description of calculations of $C F(A T)$ factors for slab, cylindrical, and spherical samples provided the SNM is uniformly distributed in the sample. Thus for a uniform sample, $C F(A T)$ can be calculated, $C F(R L)$ can be determined experimentally and then one can calculate the calibration constant $K$ from Eq. 6.

The uniformity of the distribution of SNM in a standard can be determined by comparing the measured TCR from segment to segment.

$$
T C R_{i}=R R_{i} * C F_{i}(R L) * C F_{i}(A T)
$$

If the $T C R_{i}$ for the main portion of the standard does not vary more than $10 \%$ from the average, then the distribution can be considered to be uniform. The $T C R_{i}$ at the end segment of the standard will drop to a low value or to zero in a smooth manner. An example of the TCR distribution of an SGS can standard with acceptable uniformity is shown in Fig. 8.

3. Transmission. The transmission of the gamma rays from a standard should be in a reasonable range. As an illustration of this requirement, consider the expression for $C F(A T)$ for the far-field assay of a box-shaped sample. The attenuation correction can be expressed as follows:

$$
\begin{aligned}
& C F(A T)=-\ln (T) /(1-T), \\
& \sigma(C F)=\left(\left(1-T+T^{*} \ln T\right) /(1-T)^{2}\right) * \sigma_{\mathrm{r}}(T) .
\end{aligned}
$$




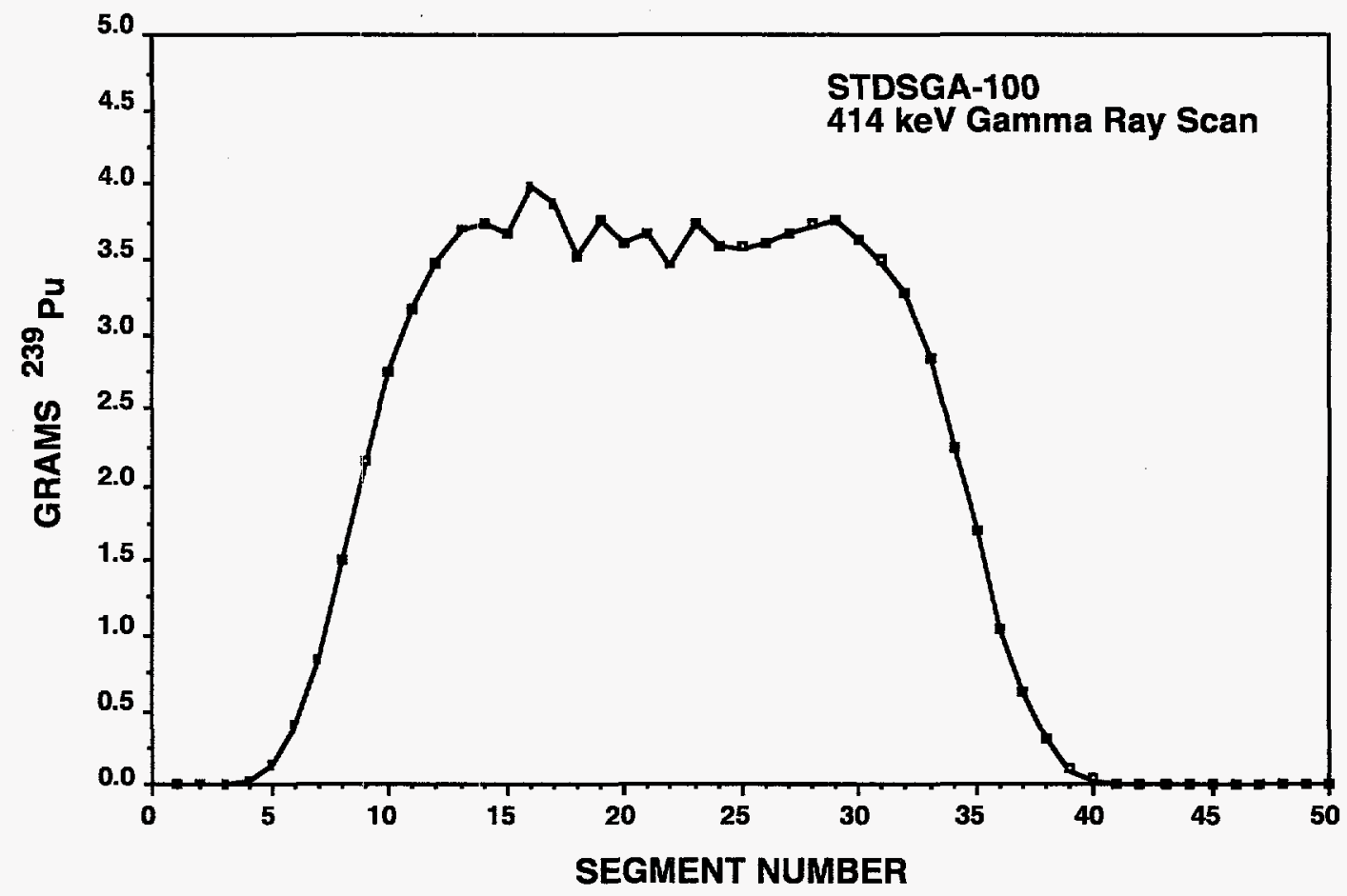

Fig. 8. Uniformity test of SGS can standard. The collimator width was $1.27 \mathrm{~cm}$ and the scanning step was $0.635 \mathrm{~cm}$. The 414-keV gamma-ray peak was used to determine the ${ }^{239} \mathrm{Pu}$ content in each segment.

As the transmission becomes very small, the $C F$ becomes large and the error of the correction also becomes very large. In general the transmission of the standard should be greater than $10 \%$ so that the assay precision will be acceptable in a reasonable counting time.

Standards for SGS are fabricated with diatomaceous earth (DE) or graphite as typical diluents. Table $\mathrm{V}$ lists the transmission through $1 \mathrm{~cm}$ of $\mathrm{DE}$ or graphite at the common assay energies of SGS, assuming the density of DE to be $0.26 \mathrm{~g} / \mathrm{mL}$ and the density of graphite to be $1.0 \mathrm{~g} / \mathrm{mL}$.

For transmission of $10 \%$ or higher at the uranium assay energy of $186 \mathrm{keV}$, graphite diluent must be less than $18 \mathrm{~cm}$ thick and DE less than $70 \mathrm{~cm}$ thick. Therefore in preparing a 200-l drum standard, DE would be a better diluent than graphite.

Table V

Transmission of Gamma Rays Through $1 \mathrm{~cm}$ of Matrix

\begin{tabular}{|l|l|l|l|l|}
\hline Energy $(\mathrm{keV})$ & $\mathrm{DE}$ & $\mathrm{DE}$ & Graphite & Graphite \\
\hline & $\mu_{\mathrm{m}}\left(\mathrm{cm}^{2} / \mathrm{g}\right)$ & Transmission & $\mu_{\mathrm{m}}\left(\mathrm{cm}^{2} / \mathrm{g}\right)$ & Transmission \\
\hline 129 & 0.1430 & 0.9635 & 0.1382 & 0.8709 \\
\hline 186 & 0.1256 & 0.9679 & 0.1246 & 0.8828 \\
\hline 279 & 0.1098 & 0.9721 & 0.1090 & 0.8967 \\
\hline 414 & 0.09381 & 0.9759 & 0.09367 & 0.9106 \\
\hline
\end{tabular}


The above calculation has only taken into account the attenuation of the matrix materials. In actual standards, especially the high-mass standards, the attenuation of the assay gamma rays in the SNM should also be included. If the plutonium standard is intended for an SGS system with a "lump correction," then the standard should be calibrated for all the assay energies: 129,279 , and $414 \mathrm{keV}$.

4. End Effects. We mentioned that the value of $T C R_{i}$ would drop toward the ends of the standard. At the "end" segments there may be some SNM mixed with diluent but not a full complement of SNM. In SGS systems, the transmission source is collimated and only a narrow beam will shine through the segment. In this "end" segment, the transmission source will sometimes shine through the SNM but sometimes will miss the SNM. The measured transmission will be higher and therefore the correction factor will be lower than is necessary to correct the attenuation within this segment. Thus the "end effects" tend to bias the assay in the negative direction.

There are ways of correcting for "end effects": generate the correction factor by using the transmission of neighboring segments where the segment is filled with SNM and diluent.

For SGS systems not equipped with the "end effect" correction, the requirements for the standard are that the number of segments filled with SNM and diluent be large compared to the end segments. Nominally this ratio should be $>10$ to reduce the bias to the $<5 \%$ level. This means the SGS standard should be tall and thin.

5. Particle Size. Figure 9 is a graph of the calculated gamma-ray selfattenuation for individual particles as a function of particle size $^{2}$ and gives the fraction of gamma rays escaping unscattered and unabsorbed from spherical sources. Table VI gives the gamma-ray leakage fraction from plutonium oxide particles of different sizes at four relevant gamma-ray energies.

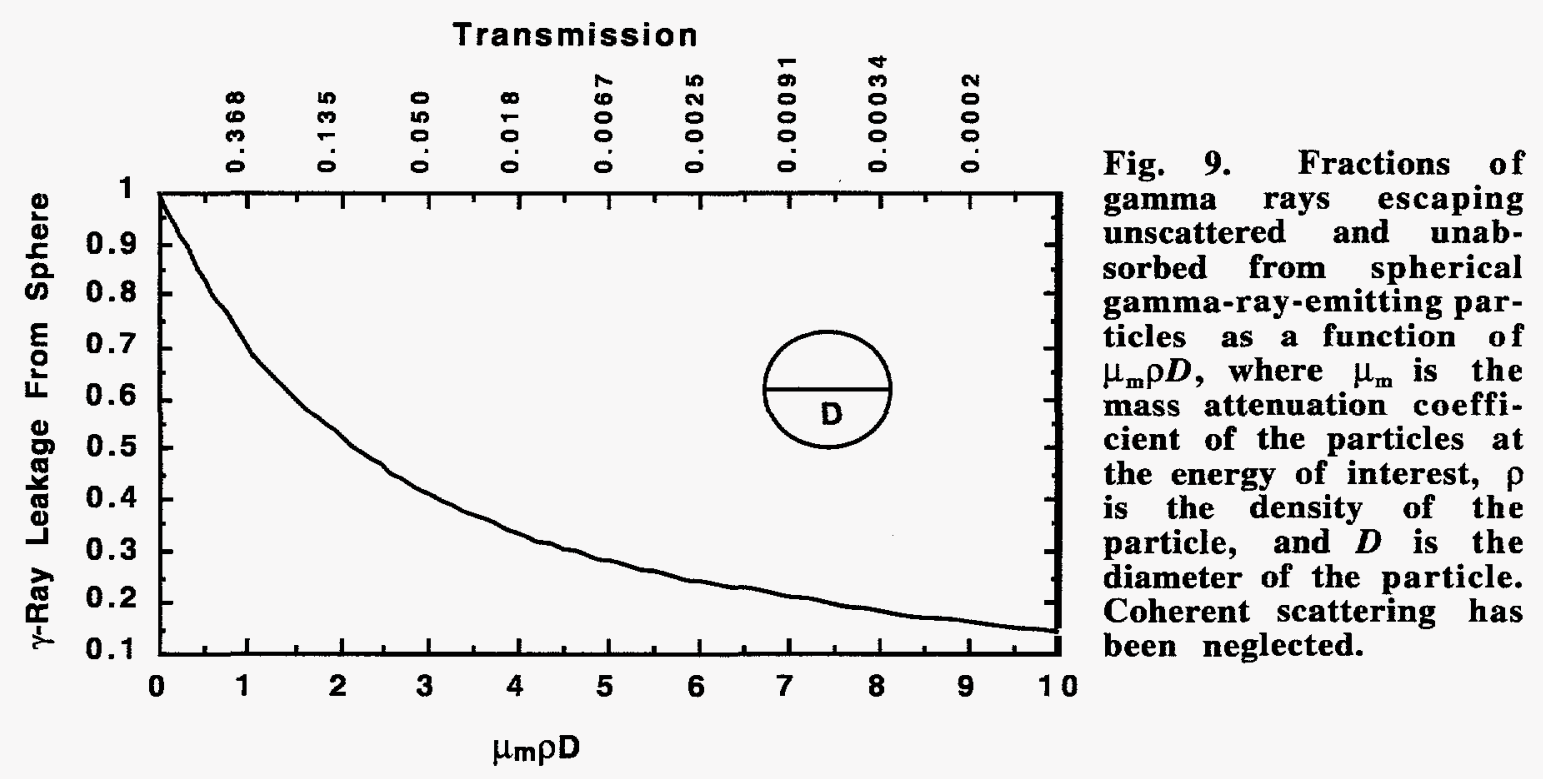


Table VI

Gamma-Ray Leakage Fraction from Plutonium Oxide Particles of Different Sizes, at Various Energies

\begin{tabular}{|c|c|c|c|c|c|}
\hline Energy (keV) & & 129 & 203 & 345 & 413 \\
\hline$\mu_{\mathrm{m}}\left(\mathrm{cm}^{2} / \mathrm{g}\right)$ & & 3.72 & 1.26 & 0.386 & 0.268 \\
\hline $\begin{array}{l}\text { Particle Size } \\
\text { (microns) }\end{array}$ & Mesh Size & & & & \\
\hline 150 & 100 & 0.818 & 0.932 & 0.978 & 0.985 \\
\hline 106 & 140 & 0.866 & 0.952 & 0.985 & 0.989 \\
\hline 90 & 170 & 0.885 & 0.958 & 0.987 & 0.991 \\
\hline 75 & 200 & 0.902 & 0.965 & 0.989 & 0.992 \\
\hline 53 & 270 & 0.929 & 0.975 & 0.992 & 0.994 \\
\hline 38 & 400 & 0.949 & 0.982 & 0.994 & 0.996 \\
\hline 20 & & 0.972 & 0.990 & 0.997 & 0.998 \\
\hline 5 & & 0.993 & 0.997 & 0.999 & 1.000 \\
\hline
\end{tabular}

The particle size can be cletermined by using an optical microscope or a suspension technique. Fig. 10 shows the particle distribution of one batch of high-fired plutonium oxide at Los Alamos. ${ }^{3}$ The majority of the particles are less than 50 microns in diameter. We also found that the oxide could only pass through a 100 to 120 mesh sieve, and it is our opinion that the oxide particles are small but they tend to agglomerate into large clumps.

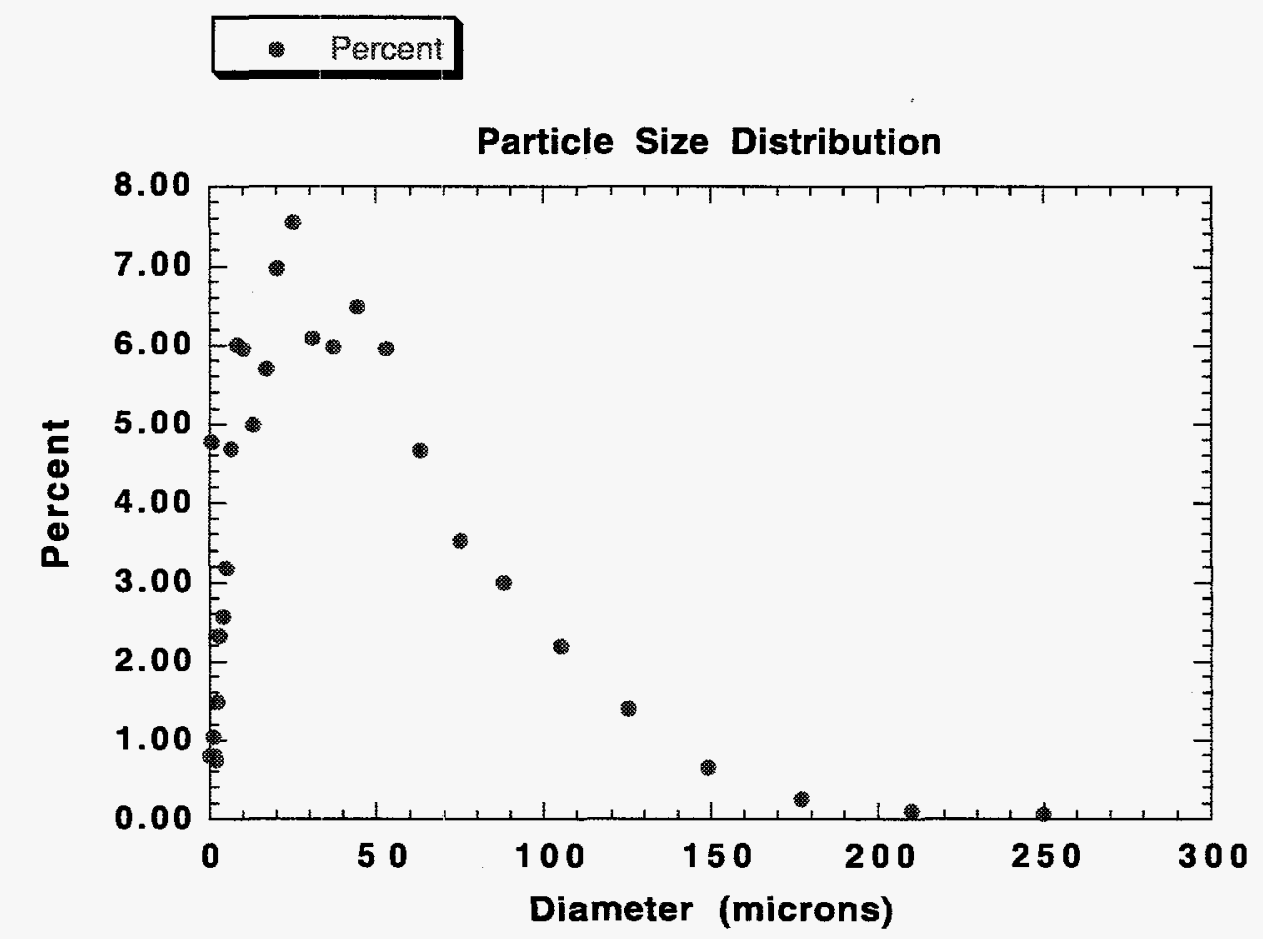

Fig. 10. Particle size distribution of high-fired plutonium oxide. 


\section{Typical Examples of SGS Standards}

Can Standard. We have prepared several sets of SGS plutonium can standards. ${ }^{4}$ These standards are contained in welded stainless steel containers (inner can diameter of $9.54 \mathrm{~cm}$ and height of $27.30 \mathrm{~cm}$; outer can diameter of $9.995 \mathrm{~cm}$ and height of $28.32 \mathrm{~cm}$ ). The mass ranges from $\sim 10 \mathrm{~g}$ to $240 \mathrm{~g}$ of ${ }^{239} \mathrm{Pu}$. After the standards were made, a consistency check was performed among these standards and the results are shown in Fig. 11. We found that the calibration generated from these standards agreed to $0.13 \%$, indicating a high degree of consistency among the standards from $10 \mathrm{~g}$ to $240 \mathrm{~g}$. This shows that for NDA systems such as SGS, where the calibration is a constant, it is possible to use only one standard for calibration. In practice it is desirable to have two to four standards, whereas in systems where the calibration is not a constant, many more standards may be needed. It is also true that with systems such as the SGS, low-mass standards are not necessary because counting statistics of low-mass standards are poor with normal counting time, and therefore they do not improve the accuracy of the calibration.

The SGS drum standards are discussed in detail in Chapter IV.

- $414 \mathrm{kev}$

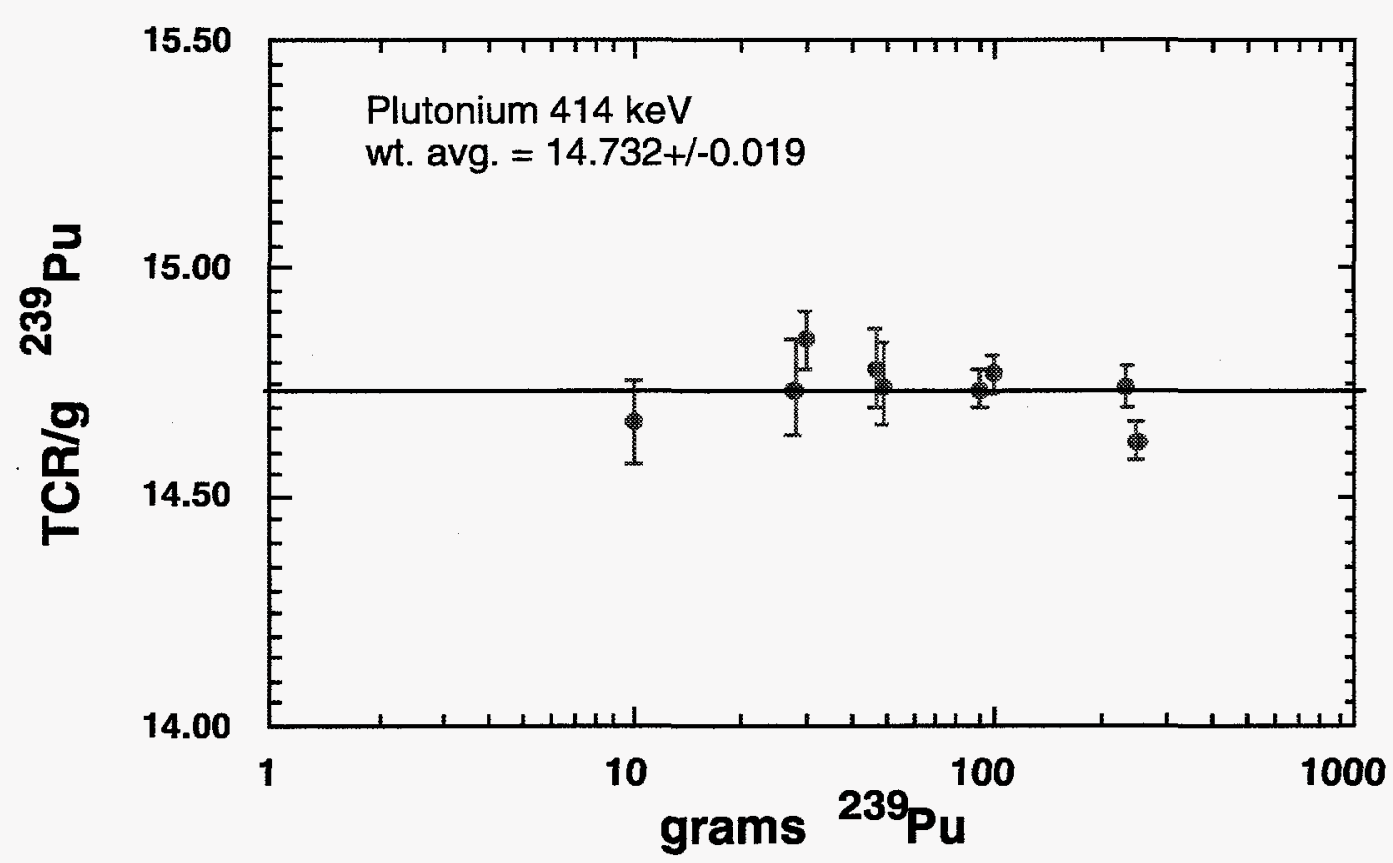

Fig. 11. Total corrected counts per gram of ${ }^{239} \mathrm{Pu}$ for can SGS standards from $10 \mathrm{~g}$ to $240 \mathrm{~g}$. 


\section{References}

1. E. R. Martin, D. F. Jones, and J. L. Parker, "Gamma-Ray Measurements with the Segmented Gamma Scan," Los Alamos Scientific Laboratory report LA-7059-M (December 1977).

2. J. L. Parker, "The Use of Calibration Standards and the Correction for Sample Self-Attenuation in Gamma-Ray Nondestructive Assay," Los Alamos National Laboratory report LA-10045, Rev. (November 1986).

3. R. Marshall, M. Foley, L. McCullough, and D. Vance, "Plutonium Working Reference Materials For the NDA PDP Program," in "Proceedings 4th Nondestructive Assay and Nondestructive Examination Waste Characterization Conference," Salt Lake City, Utah, October 24-26, 1995, Idaho National Engineering Laboratory report CONF-951091.

4. S.-T. Hsue, S. M. Simmonds, V. L. Longmire, and S. M. Long, "Design and Fabrication of SGS Plutonium Standards," in Fourth International Conference on Facility Operations-Safeguards Interface (American Nuclear Society, La Grange Park, Illinois, 1991), pp. 410-413. 


\section{OVERVIEW: NEUTRON AND CALORIMETER MEASUREMENTS}

\section{A. Neutron Techniques}

\section{Bulk Measurements of Uranium and Plutonium Masses}

a. Thermal Neutron Coincidence Counting. ${ }^{1-4}$ Thermal neutron coincidence counters (TNCCs) are used extensively for NDA of bulk nuclear material. They use ${ }^{3} \mathrm{He}$ proportional counters moderated by polyethylene. Fast neutrons from the sample are slowed in the polyethylene and captured in the ${ }^{3} \mathrm{He}$. TNCCs are useful primarily because fast neutrons penetrate the sample well and because time-correlated neutrons are directly related to the fission process and thus to the nuclear material content. Special pulse-processing electronics enable discrimination of time-correlated neutrons from random neutron pulses. TNCCs are used in passive mode to assay the even, spontaneously fissioning isotopes of plutonium $\left({ }^{238} \mathrm{Pu},{ }^{240} \mathrm{Pu}\right.$, and ${ }^{242} \mathrm{Pu}$ ) and in active mode to assay induced-fissionable isotopes, primarily ${ }^{235} \mathrm{U}$, in bulk samples. In passive mode, no external neutron sources are required. In active mode, AmLi sources are typically used to induce fissions in ${ }^{235} \mathrm{U}$.

Passive Mode for Plutonium Passive TNCCs are well suited for measuring dense plutonium-bearing materials, such as pure metal and oxide, over a wide range of masses. Precision and accuracy are much better for pure materials than for scrap. For pure plutonium oxide, there are two primary sources of neutrons and one secondary source. The two primary sources are spontaneous fission and $(\alpha, n)$ reactions. Several plutonium isotopes decay by prolific emission of alpha particles, some of which undergo reactions with oxygen to produce single neutrons. The secondary source of neutrons in plutonium oxide is fast-neutron induced fission, occurring in all plutonium isotopes. For pure plutonium metal, the $(\alpha, n)$ neutron source is zero because of the absence of low-Z elements.

For plutonium scrap, all three sources of neutrons exist as with plutonium oxide; however, the $(\alpha, n)$ component is calculable for pure oxide, but not for scrap, unless an impurity analysis is available. Table VII below summarizes primary and secondary neutron sources and their origins for plutonium metal, oxide, and scrap.

Table VII

Neutron Sources from

Plutonium Metal, Oxide, and Scrap

\begin{tabular}{|c|c|c|c|}
\hline & Spontaneous Fission & Induced Fission & $(\alpha, \mathbf{n})$ Reactions \\
\hline Pure Metal & $\begin{array}{c}{ }^{238} \mathrm{Pu},{ }^{240} \mathrm{Pu}, \\
\text { and }{ }^{242} \mathrm{Pu}\end{array}$ & ${ }^{238} \mathrm{Pu}$ through ${ }^{242} \mathrm{Pu}$ & none \\
\hline Pure Oxide & $\begin{array}{c}{ }^{238} \mathrm{Pu},{ }^{240} \mathrm{Pu}, \\
\text { and }{ }^{242} \mathrm{Pu}\end{array}$ & ${ }^{238} \mathrm{Pu}$ through ${ }^{242} \mathrm{Pu}$ & oxygen \\
\hline $\begin{array}{c}\text { Impure Oxide } \\
\text { and Scrap }\end{array}$ & $\begin{array}{c}{ }^{238} \mathrm{Pu},{ }^{240} \mathrm{Pu}, \\
\text { and }{ }^{242} \mathrm{Pu}\end{array}$ & ${ }^{238} \mathrm{Pu}$ through ${ }^{242} \mathrm{Pu}$ & $\begin{array}{c}\text { oxygen plus other low-Z } \\
\text { impurities, e.g., fluorine }\end{array}$ \\
\hline
\end{tabular}


For passive assay of plutonium materials based on conventional thermal neutron coincidence counting, calibration is performed by using standards to determine a curve with the real coincidence count rate $K$ plotted versus the ${ }^{240} \mathrm{Pu}$ (effective) mass. ${ }^{240} \mathrm{Pu}$ (effective) mass contains contributions from ${ }^{238} \mathrm{Pu}$ and ${ }^{242} \mathrm{Pu}$ : it is the mass of ${ }^{240} \mathrm{Pu}$ that would give the same coincidence response as that obtained from all the even plutonium isotopes in the sample.

If there were no induced fission in the sample, the calibration curve of $R$ versus the ${ }^{240} \mathrm{Pu}$ (effective) mass would be linear and preparation of standards would be relatively simple. The effects of variations in impurities, geometry, and density would be relatively unimportant. However, the existence of induced fission (multiplication) in the sample causes these effects to be important considerations in preparing standards for and calibrating TNCCs.

Figure 12 shows two calibration curves for a set of pure plutonium oxide standards. The upper $R$ curve is uncorrected. The lower curve is corrected for multiplication using a method described below. The lower curve contains contributions from spontaneous fission only.

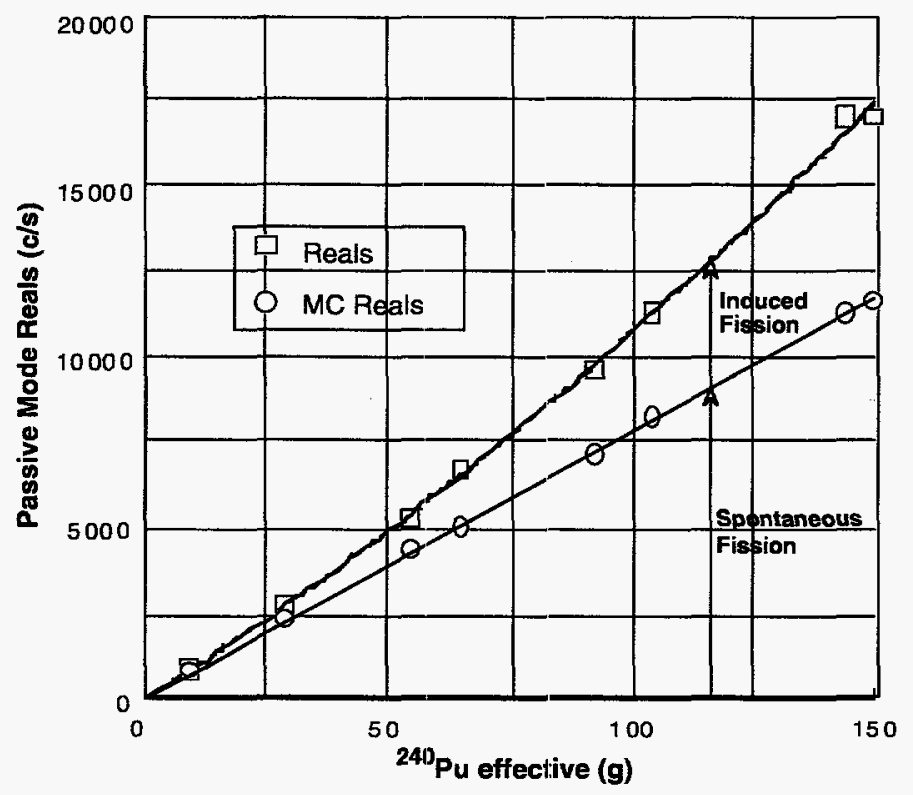

Fig. 12. Calibration curves for passive neutron coincidence counting of pure plutonium oxide. Upper curve is the real coincidence count rate (Reals) versus the ${ }^{240} \mathrm{Pu}$ effective mass. Lower curve (MC Reals) is corrected for induced fission or multiplication.

Fissions may be induced in the item to be assayed by primary neutrons born in spontaneous fission and $(\alpha, n)$ reactions or by secondary neutrons from other induced fissions. For a given mass of ${ }^{240} \mathrm{Pu}$ (effective), the number of induced fissions, and therefore the contribution to $R$, depends on $(\alpha, n)$ reactions in the sample and also on plutonium isotopic composition, geometry, and density. The self-multiplication of the item (ratio of primary plus secondary to primary neutrons) increases with plutonium mass and density.

For pure plutonium oxide, or impure plutonium oxide with known impurities, there are three sources of neutrons, but the ratio of $(\alpha, n)$ to spontaneous fission neutron production $(\alpha)$ is known. Therefore, in this case, there are two unknown sources of neutrons and two measured parameters, $R$ and $T$. Solving these two equations results in a 
"multiplication-corrected" $R$ that is used for calibration and assay. This "known- $\alpha$ " method is preferable to the uncorrected- $R$ calibration for pure materials or for impure materials with known impurities because variations in $R$ due to variations in induced fission are removed. The upper curve of Fig. 12 contains these variations. Precision is improved with the "known- $\alpha$ " method because both $R$ and $T$ are used in the analysis, and the measurement of $T$ is more precise than $R$. For most impure plutonium materials for assay, however, impurity levels are unknown and the method is usually restricted to pure materials (metal and oxide). In cases where geometry and density are constant for a set of impure items, a calibration of multiplication $M$ versus ${ }^{239} \mathrm{Pu}$ (effective) mass can be used to eliminate one of the three unknown sources of neutrons. This is the "known- $\boldsymbol{M}$ " method and is useful in some cases.

\section{Guidelines for Standards Preparation}

- As with all NDA methods, standards must span the mass range of the items for passive TNCC assays uncorrected for sample self-multiplication, where three to five standards are recommended. For the "known- $\alpha$ " calibration, in principle, only one or two standards are required to define the linear calibration. For the "known- $\boldsymbol{M}$ " calibration, approximately five standards are necessary to define the relationship between item self-multiplication and ${ }^{239} \mathrm{Pu}$ (effective) mass.

- In preparing bulk plutonium standards for conventional, passive TNCC assay, it is crucial to accurately characterize them with regard to low- $\mathrm{Z}$ impurity analyses.

- To minimize measurement bias for pure metal or oxide, standards should be free of low- $Z$ impurities. For pure materials, the "known- $\alpha$ " assay method is preferred because it is insensitive to parameters that affect induced fission, i.e., plutonium composition, geometry, and density. Therefore, the standard set need not be uniform with respect to these parameters.

- For impure materials, concentrations of low-Z impurities in the standards should be the same as for the unknowns because the two measured quantities used for assay in conventional thermal neutron coincidence counting, the total neutron count rate $T$ and the real coincidence count rate $R$, both depend on $(\alpha, n)$ neutron emission.

- For impure materials to be assayed by passive TNCCs, the standards should represent the unknowns with regard to low- $Z$ impurities, plutonium composition, geometry, and density. These four factors affect induced fission and can introduce biases in $R$ and $T$.

- TNCC standards should not contain matrix or packaging, e.g., hydrogenous materials, that significantly change the average energy of emitted neutrons. TNCCs count low-energy neutrons after energy degradation by a neutron moderator (typically polyethylene). TNCC detector heads are designed to be insensitive to variations of emitted neutron energy. Alternatively, standards should contain the same moderating materials as the unknowns.

- The use of Monte Carlo simulations can markedly reduce the number of physical standards required for adequate passive TNCC calibrations. 
Calibration parameters have been determined to an accuracy of $\sim 1 \%$ for pure materials in well-controlled geometries, e.g., reactor fuel assemblies.

\section{Los Alamos Plutonium Oxide Standards}

A set of eight plutonium oxide standards ("LAO series"), with $\sim 16 \%{ }^{240} \mathrm{Pu}$, ranging in mass from 60 to $870 \mathrm{~g}$ of plutonium, was prepared in 1983 for passive TNCC. The LAO series is well-characterized and considered "pure" in terms of low- $Z$ contaminants and water $(<0.5 \%)$. The LAO set is well-characterized with regard to plutonium mass, isotopics, low-Z impurities, and moisture. The standards are doubly contained in welded steel food-pack cans. They have been used successfully for calibration of passive TNCCs used at Los Alamos, Savannah River, LLNL, Hanford, and RFETS.

Active Mode for Uranium Active TNCCs are well-suited for measuring a wide range of uranium-bearing materials, such as $\mathrm{HEU}$ metal, uranium-aluminum plates and billets, uranium-thorium-carbon beads and pebbles, $\mathrm{U}_{3} \mathrm{O}_{8}$ powder, $\mathrm{UF}_{4}, \mathrm{UO}_{2}$, and reactor fuel elements. In principle, each of these material categories requires a separate calibration using standards that properly span the mass range of contained ${ }^{235} \mathrm{U}$ and are as nearly identical to the unknowns as possible.

For most applications, a single active measurement is made using AmLi isotopic neutron sources. AmLi produces neutrons through $(\alpha, n)$ reactions with an average neutron energy of $\sim 300 \mathrm{keV}$. The active measurement produces coincidence counts primarily from induced fission in ${ }^{235} \mathrm{U}$, but a small component of counts from spontaneous fission in ${ }^{238} \mathrm{Pu}$ can exist. In some fresh reactor fuel elements, this component is large enough to warrant a separate passive measurement.

These counters are operated in two modes: fast and thermal. In fast mode, cadmium lines the sample cavity producing an interrogating neutron flux that is free of neutrons below the cadmium cutoff $(\sim 0.3 \mathrm{eV})$. With cadmium removed, thermal neutrons are responsible for most of the induced fissions in ${ }^{235} \mathrm{U}$. Fast mode is generally preferred because of more thorough sample interrogation and lessened sensitivity to the matrix. However, in thermal mode, masses as low as $1 \mathrm{~g}$ of ${ }^{235} \mathrm{U}$ can be detected in $1000 \mathrm{~s}$, whereas, the 1000 -s detection limit for fast mode is $100-200 \mathrm{~g}$ of ${ }^{235} \mathrm{U}$.

Figure 13 is a fast-mode calibration curve for uranium oxide. The data are best fitted using a cubic equation because there are two regions of curvature; the self-shielding region extending up to $\sim 500 \mathrm{~g}$ of ${ }^{235} \mathrm{U}$ and the multiplication region beyond. In the selfshielding region, some neutrons are shielded from the interior of the item and cannot induce fissions. In the multiplication region, fissile mass is large enough for induced fission to produce more neutrons than are lost in self-shielding. Figure 13 contains more calibration data than is needed to adequately define the curve's shape. The data below $\sim 150 \mathrm{~g}$ of ${ }^{235} \mathrm{U}$ were taken with very long count times to obtain adequate precision. In practice, the calibration would be used to assay between 150 and $900 \mathrm{~g}$. Only a few standards are required to define the calibration in this range.

Figure 14 is a thermal-mode calibration curve for uranium oxide powder. The calibration items are the same as in Fig. 13 up to $\sim 100 \mathrm{~g}$ of ${ }^{235} \mathrm{U}$. Note that the coincidence response is much higher (by approximately a factor of 25) for thermal mode than for the fast mode. 


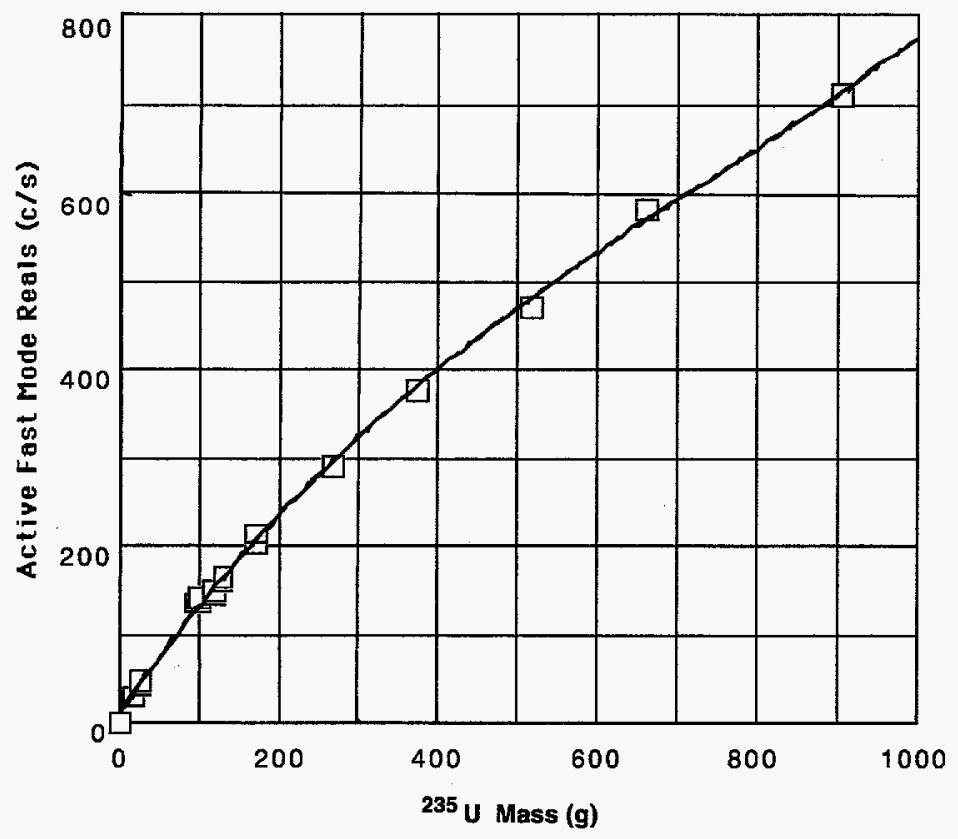

Fig. 13. Calibration curve for neutron coincidence counting of uranium oxide powder in the active fast mode. Items are each nominally $1 \mathrm{~kg}$ of oxide, with variable enrichment $(\sim 1 \%$ to $90 \%$ ).

Fig. 14. Calibration curve for neutron coincidence counting of uranium ox ide. Items are each nominally $1 \mathrm{~kg}$ of oxide in the active thermal mode, with variable enrichment $(\sim 1 \%$ to $20 \%$ ).

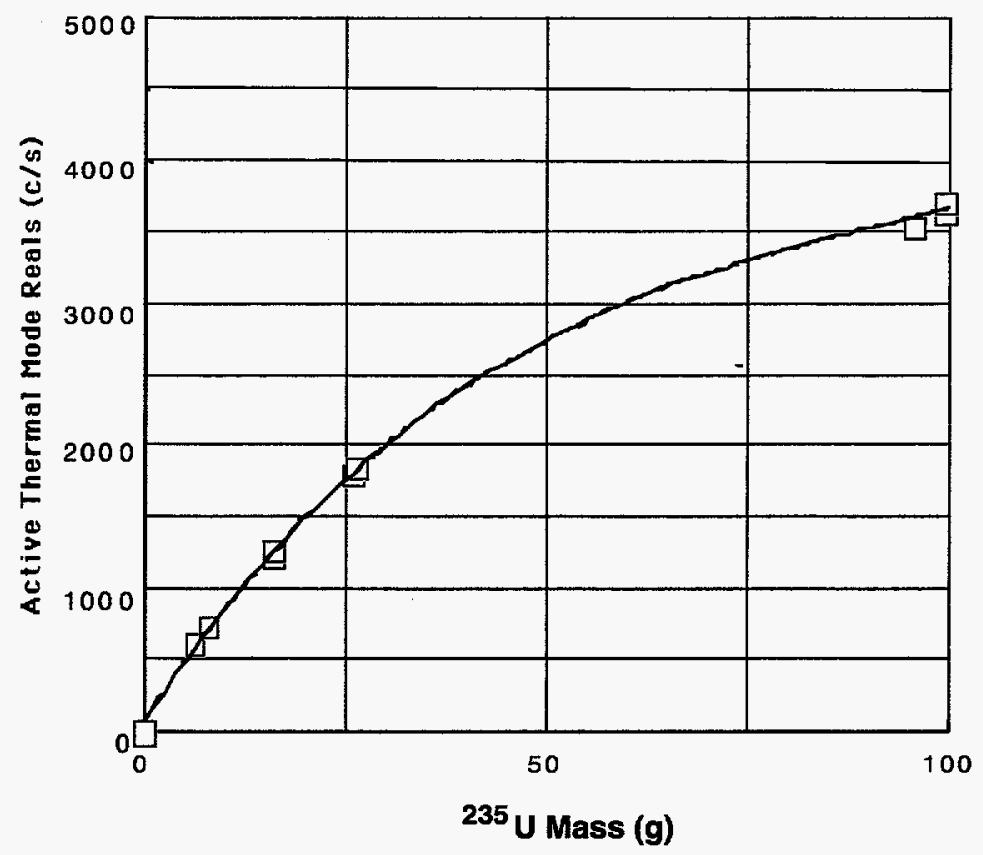

Only the self-shielding region is evident in Fig. 14. The thermal calibration is not usually carried beyond $100 \mathrm{~g}$ of ${ }^{235} \mathrm{U}$ because the curve becomes too flat, introducing large assay errors.

Note the calibration shown in Fig. 14 is only useful for assays between $\sim 5$ and $30 \mathrm{~g}$. The gap between 25 and $90 \mathrm{~g}$ is too large. The complete data set is shown to illustrate the self-shielding curvature. 


\section{Guidelines for Standards: Preparation}

- Generally, for active TNCC assays, standards must be as representative of the unknowns as possible with regard to ${ }^{235} \mathrm{U}$ density, geometry, and matrix because response is directly related to the induced fission rate, which can be influenced by all of these parameters. This statement is especially true for thermal-mode assay because thermal neutron penetration is very weak in most items of interest and strongly dependent on the absorbing materials present in the matrix.

- As with passive TNCC assay, the use of Monte Carlo simulations can markedly reduce the number of physical standards required for adequate, active TNCC calibrations. Once a calibration curve shape has been determined for a material type using simulations, the curve can be tied to a particular detector by measuring only one or a few physical standards.

\section{Los Alamos Uranium Standards}

The UISO series of high-quality uranium oxide standards consists of eight items, all with $990 \mathrm{~g}$ of uranium, ranging in enrichment from $11 \%-91 \%$. The standards were originally to be uranium enrichment standards, but they also serve as active TNCC standards. Calibration measurements of these standards are shown in Figs. 12 and 13.

Another excellent standard set ("C20 series") for active TNCC is composed of 93\% enriched uranium metal disks: seven with $\sim 500 \mathrm{~g}$ of uranium and two with $\sim 260 \mathrm{~g}$ of uranium. The disks are nickel-plated, well characterized, and quite uniform.

The UISO and C20 standard series have been used extensively to calibrate active TNCCs used for inventory verification measurements at Los Alamos, SRS, LLNL, Portsmouth, and ORNL DOE facilities.

\section{b. Thermal Neutron Multiplicity Counting. ${ }^{\text {5-6 }}$ Thermal neutron} multiplicity counters (TNMCs) are similar in design and construction to TNCCs: they both use polyethylene-moderated ${ }^{3} \mathrm{He}$ proportional counters. However, multiplicity counters are designed to maximize counting efficiency. In addition to measuring the total count rate $T$ ("singles") and the real coincidence count rate $R$ ("doubles"), multiplicity counters also measure the third moment ("triples") of the neutron multiplicity distribution. While $T$ and $R$ are proportional to efficiency and the efficiency squared, respectively, the triples rate is proportional to the cube of the neutron counting efficiency. The desire to maximize the precision of the triples measurement leads to high-efficiency detectors.

TNMCs were developed to provide three measured parameters for assay of impure plutonium-bearing materials. As mentioned previously, these materials have three sources of neutrons to be resolved: spontaneous fission, induced fission and $(\alpha, n)$ reactions. Multiplicity analysis involves solution of three equations for the singles, doubles, and triples in the three unknowns to yield the ${ }^{240} \mathrm{U}$ (effective) mass. The effects of variable multiplication and $(\alpha, n)$ impurities are removed from the assay. 


\section{Guidelines for Standards Preparation}

- Standards for TNMC assay are only required in a few instances. They are required to verify proper setting of a few detector parameters used in the multiplicity analysis. The concept of generating a calibration curve of instrument response versus ${ }^{240} \mathrm{U}$ (effective) mass does not apply to TNMC assay.

- Initial calibration of a TNMC can be done with $\mathrm{a}^{252} \mathrm{Cf}$ source alone. However, in practice, it is best to use a well-characterized pure or impure plutonium oxide standard to verify detector parameters. The calibration can then be used to assay items with a wide range of impurity concentrations, plutonium isotopes, bulk density, fissile mass, and geometry.

\section{References}

1. H. O. Menlove and J. E. Swansen, "A High-Performance Neutron Time Correlation Counter," Nuclear Technology 71, 497-505 (November 1985).

2. H. O. Menlove, "Description and Operation Manual for the Active Well Coincidence Counter," Los Alamos Scientific Laboratory report LA-7823-M (May 1979).

3. H. O. Menlove and J. E. Stewart, "A New Method of Calibration and Normalization for Neutron Detector Families," Los Alamos National Laboratory report LA-11635-M (July 1989).

4. J. E. Stewart, et al., "A Versatile Passive/Active Neutron Coincidence Counter for In-Plant Measurements of Plutonium and Uranium," in Proc. 13th ESARDA Symposium on Safeguards and Nucl. Mater. Manage. (ESARDA, Ispra, Italy, 1991), ESARDA 24, pp. 317-323.

5. M. S. Krick and J. E. Swansen, "Neutron Multiplicity and Multiplication Measurements," Nuclear Instruments and Methods 219, 384-393 (1984).

6. H. O. Menlove, et al., "Plutonium Scrap Multiplicity Counter Operation Manual," Los Alamos National Laboratory report LA-12479-M (January 1993). 


\section{Californium Shuffler}

a. Measurement Principles. A shuffler ${ }^{1,2}$ performs passive and active neutron (PAN) assays for fissile materials. The passive assays for plutonium are done in the same manner as other instruments specially designed for passive assays only, so the standards for passive assays will not be discussed here.

Active assays are done on uranium (or any other fissile material) by repeatedly irradiating the item with neutrons from a ${ }^{252} \mathrm{Cf}$ source and quickly withdrawing the source so that delayed neutrons can be counted. (Delayed neutrons are emitted by fission fragments seconds to minutes after a fission.) Typical assay times range from $10 \mathrm{~min}$. to 16 min., including a 3 or 4 min. background count and 20 to 35 "shuffles" of the ${ }^{252} \mathrm{Cf}$ source. Materials assayed in shufflers have ranged from milligrams of uranium waste to kilograms of purified uranium. (Reference 2 has illustrations of most of the shufflers built by Los Alamos.)

Mixtures of uranium and plutonium can be assayed on PAN shufflers by using both passive and active assays, but this introduces no new demands for standards than the two assays individually, so there will be no further discussion of this case.

A shuffler often has features to help characterize the items being assayed, correct for matrix effects, and help select the proper calibration standards. So called "flux monitors" can be very helpful in quantifying the amount of hydrogen in a matrix; they are a standard feature of shufflers for 55-gal. waste drums. Flux monitors are low-efficiency neutron detectors. Two are placed near the item: one wrapped in cadmium and one "bare." They detect neutrons during the irradiation of the drum by the ${ }^{252} \mathrm{Cf}$ source. The bare detector responds to the flux of nearly thermal neutrons that leave the drum, and hence to the amount of hydrogen in the drum. The cadmium-covered tube is less affected by the hydrogen and mainly indicates the strength of the ${ }^{252} \mathrm{Cf}$ source. The ratio of the responses from these two detectors is an indicator of how moderation within the drum is affecting the delayed neutron count rate. Hydrogen is very effective at moderating the energies of neutrons and can change the measured count rate for a given fissile mass. A calibration based on good standards without hydrogen can give erroneous results for a drum containing hydrogen (paper or rubber gloves), but a correction based on the flux monitor response ratio can greatly reduce the error.

The density of the material in a container is sometimes estimated by the transmission of ${ }^{252} \mathrm{Cf}$ neutrons through the container. If the density is found to be much larger than that used in the calibration, the standards used in the calibration may be inadequate and either the container should be repackaged or a new, more dense, standard developed.

So if a container has a poorly known matrix, it can be possible to characterize the hydrogen content, which is the single most important feature of the matrix to understand. This would point toward the best calibration standards for the material.

b. Physical Criteria for Standards. In the ideal world, the nature of standards is very simple: standards are identical in all important respects to the assay items and span the mass range of the fissile material. In reality, compromises and approximations are usually needed. 
Neutrons from the ${ }^{252} \mathrm{Cf}$ source must reach the item being assayed. Often matrix material affects neutron transport; the most important matrix element is hydrogen because it changes the energy of the neutrons more rapidly than any other element. Neutron absorbers are only important in the presence of hydrogen because their absorption is strong only for thermal (or nearly thermal) neutrons. An ideal standard would have the same matrix as the item to be assayed.

Neutrons that reach the item would ideally flood the volume of the item uniformly. For most masses of fissile material the neutron flux is smaller near the center of the volume because the outer portion absorbs neutrons; this is called self-shielding. An ideal standard would have the same self-shielding as the item to be assayed.

Delayed neutrons from the fission fragments must reach the neutron detector tubes surrounding the assay chamber. The matrix that affects the ${ }^{252} \mathrm{Cf}$ neutrons is equally important in this case also, so again an ideal standard would have the same matrix as the item to be assayed.

An ideal standard is stable in composition and form, despite handling or aging.

c. Preparation of Practical Standards. The ideal standard can be nearly reached in many cases, but in many others it cannot. Where items to be assayed are nearly all identical (e.g., uranium-aluminum billets ${ }^{3}$ ) a careful physical and chemical analysis can be done on a subset of the items that become the standards.

A standard may have obvious differences with the assay items that are unimportant to shuffler assay. For example, metals such as iron generally have minor effects $(<1 \%)$ on shuffler assays because they scatter neutrons without changing the energy spectrum very much; a standard and an assay item can have widely different amounts of such metals without greatly affecting the usefulness of the standard. [However, iron mixed with a moderator (e.g., paper) might have larger effects because low-energy neutrons are more affected by the iron than neutrons with the relatively high-energy spectrum from ${ }^{252} \mathrm{Cf}$.]

Standards for waste drums are less than ideal but still adequate. The exact nature (mass, chemical composition, density, or hydrogenous materials nearby) of the fissile material in a waste drum is rarely known and standards are thus only approximations. The drop in accuracy of assays for these materials is not as serious as it would be for billets that will be extruded into fuel assemblies for reactor cores. A relatively large error in a small waste quantity is still a small quantity. Standards that have been used for waste have often been a large number of small capsules, each containing 0.1 to $5 \mathrm{~g}$ of uranium; the uranium density is made sufficiently low to make self-shielding a minor problem. Using different numbers of these capsules leads to calibrations over a wide range of uranium masses (from zero to hundreds of grams).

A lack of nearly ideal standards may force the use of the best available standards combined with reasoned adjustments of the measurement results for the differences between the standards and the assay items. A nitric acid solution of uranium has been substituted for a hexone solution ${ }^{4}$ in the laboratory for safety reasons with little change in performance; the minor differences could be calculated with a Monte Carlo code. At the other extreme, using 5-in.-diameter cans of $\mathrm{U}_{3} \mathrm{O}_{8}$ powder as standards for assays of thin, dense reactor fuel pins of uranium and plutonium requires a large adjustment to the measurement based on Monte Carlo calculations; such a large adjustment is likely to introduce large uncertainties to the final result. 
Where standards are lacking entirely, the calibration results from another shuffler of the same (or nearly the same) design can be used, but some bias can be expected. For example, there are five shufflers for 55-gal. drums built from the same blueprints (and a sixth with nearly the same design). The same $\mathrm{U}_{3} \mathrm{O}_{8}$ powder standards were used on two of these shufflers and the results were applied to a third shuffler where standards were not available. The main problem in doing this is the uncertainty in the relative emission rates of the ${ }^{252} \mathrm{Cf}$ sources; a source fabricator's stated emission rates could easily be in error by $10 \%$ or more. So an unknown bias is introduced in the third shuffler that could be eliminated if any single item could be measured on this shuffler and either of the other two; this item would be a working standard that need not be well characterized.

d. Examples of Standards. Table VIII shows the various assay items encountered to date by shufflers and the practical standards used in connection with them. The list of items starts with those having the best standards and progresses toward those with the poorest standards.

Table VIII

Assay Items and Shuffler Standards

\begin{tabular}{|l|l|}
\hline \multicolumn{1}{|c|}{ Assay Item } & \multicolumn{1}{c|}{ Standard } \\
\hline Uranium-aluminum billets & Physically sampled and chemically analyzed billets \\
\hline Uranium scrap metal & Scrap metal of the same type with chemical analysis \\
\hline Uranium oxide cans & Nearly identical cans with chemical analysis of samples \\
\hline Pellets of UF & Similar pellets with chemical analysis \\
\hline Baskets of leached hulls & Specially prepared baskets with known amounts of fissile materials \\
\hline $\begin{array}{l}\text { Flowing liquid waste from spent } \\
\text { fuel reprocessing }\end{array}$ & $\begin{array}{l}\text { Laboratory development: similar solutions that are chemically less } \\
\text { hazardous and without fission products, with Monte Carlo } \\
\text { simulations to account for differences with the real solutions; known } \\
\text { flow rates }\end{array}$ \\
\hline $\begin{array}{l}\text { Spent fuel assemblies } \\
\text { Plant calibration: Specially prepared solutions with known } \\
\text { concentrations of the fissile material and known flow rates }\end{array}$ \\
\hline $\begin{array}{l}\text { 55-gal. drums of waste in known } \\
\text { categories of matrices }\end{array}$ & $\begin{array}{l}\text { Simulated spent fuel assemblies using a homogeneous mixture of } \\
\text { simulated fissile material and matrix (zirconium oxide) }\end{array}$ \\
\hline $\begin{array}{l}\text { 55-gal. drums of waste with poorly } \\
\text { known matrices }\end{array}$ & $\begin{array}{l}\text { 55-gal. drums fabricated with nearly identical matrices and with } \\
\text { known amounts of the same fissile materials }\end{array}$ \\
\hline $\begin{array}{l}\text { Assorted inventory items with a } \\
\text { wide variety of compositions and } \\
\text { matrices } \\
\text { matrices. Features of the shuffler are used to help characterize the } \\
\text { waste and correct the measurement }\end{array}$ & $\begin{array}{l}\text { It is impractical to have standards that are very similar to these many } \\
\text { different items. Use a small number of standard types (perhaps only } \\
\text { one); fundamental nuclear information or Monte Carlo simulations } \\
\text { can be used to estimate the adjustment of the data for the calibration } \\
\text { standards available }\end{array}$ \\
\hline
\end{tabular}


The items assayed in shufflers are diverse. All types and amounts of fissile materials may be encountered and the packaging matrices can be very important. It is impractical to propose a small number of standards that would be adequate for all shufflers and items. But for any one shuffler at a facility, the materials to be assayed can be placed into categories sharing the same standards; the smaller the number of categories at a facility, the simpler the problem with standards.

The simplest case is when the shuffler is to assay only one material type (e.g., uranium in liquid waste flowing through a pipe or nearly-identical cans of process $\mathrm{UO}_{2}$ ). It is practical for a facility to prepare and characterize a small number of standards that closely match the material to be measured.

The type of material may be limited but the mass range might be extensive (e.g., in uranium-aluminum scrap or billets ${ }^{3}$ ), so in this case a larger number of standards would be needed to include the expected assay range. An alternative would be to have only one or a few standards and extend the calibration range with a Monte Carlo simulation of the shuffler, using the existing standards to benchmark the calculations.

The type of material may be limited in nature, but diverse in quantity and embedded in a wide range of matrices; waste drums are an example. Instead of fabricating a huge number of standards, it is possible to fabricate a number of capsules each with a small amount of the fissile materials and a number of containers with the various matrices of interest. By using different numbers of these fissile-bearing capsules within the assorted matrices, many different cases can be prepared one at a time and a particular case can be recreated at any time to check the calibration. A standard can be prepared quickly with this modular approach to match a given matrix, fissile loading, and fissile distribution. ${ }^{2}$

When a shuffler is used to help verify an inventory of miscellaneous items, whatever standards are used are unlikely to closely match many of the items. The only practical recourse is to use Monte Carlo simulations to calculate correction factors for the differences in shapes and contents.

Some rather generic features of standards apply here also. Stability with time and handling are always important and usually met easily by shuffler standards. Stable forms of uranium are normally used in sealed containers. However, experience with cans of $\mathrm{U}_{3} \mathrm{O}_{8}$ powder has shown that uncontrolled handling will change the density of the powder and its shape within the can enough to cause variations in the assay results beyond the usual statistical fluctuations, particularly when the oxide fills only a small fraction of the can. A standard handling procedure controls this variation very well. But it is best to have standards with more stable configurations. Metals are stable because they are rigid solids; low-density standards have been prepared from solutions dispersed and absorbed onto a substrate (such as alumina). ${ }^{2}$

\section{References}

1. P. M. Rinard, "Shuffler Instruments for the Nondestructive Assay of Fissile Materials," Los Alamos National Laboratory report LA-12105-MS ( May 1991). 
2. P. M. Rinard, E. L. Adams, H. O. Menlove, and J. K. Sprinkle, Jr., "The Nondestructive Assay of 55-Gallon Drums Containing Uranium and Transuranic Waste Using Passive-Active Shufflers," Los Alamos National Laboratory report LA-12446-MS (November 1992).

3. P. M. Rinard, E. T. Sadowski, and F. G. Armstrong, "Billet Shuffler Calibration Data and Interpolation Schemes," Los Alamos National Laboratory report LA12351-MS (July 1992).

4. P. M. Rinard, T. W. Crane, T. Van Lyssel, K. E. Kroncke, C. M. Schneider, and S. C. Bourret, "A Delayed-Neutron Monitor for a Liquid-Waste Stream with High Gamma-Ray Intensity," in Proc. of the International Topical Meeting on Safety Margins in Criticality Safety, (American Nuclear Society, Inc., La Grange Park, Illinois, 1989), pp. 158-160. 


\section{B . Calorimetry}

1. Principles. The calorimetric assay of radioactive materials uses the thermal power generated by radioactive decay to determine the mass of SNM inside a container. In the most widely used version of this technique, the heat generated in the sample is detected by a temperature-sensitive wire heat sensor, hundreds of feet in length, wrapped around a cylindrical sample chamber. The sample to be measured is placed in the chamber and covered with an insulated plug. The heat flow out of the container is measured by the heat sensor. The heat sensor is placed so that it detects a representative portion of all the heat flowing out of the sample. As a result, the calorimetry measurement is independent of sample properties such as matrix density, chemical composition, SNM inhomogeneity, specific heat, or thermal conductivity. For most accountable nuclear materials with appreciable thermal power, the calorimeter detects nearly $100 \%$ of the decay energy transformed into heat.

To measure the quantity of SNM or tritium in a sample, the heat generated by the sample and the specific power $(\mathrm{W} / \mathrm{g})$ of the radioactive isotopes in the sample have to be determined. For multi-isotopic samples, the specific power can be determined by isotopic measurements and basic nuclear decay constants. Only the decay constants are needed for mono-isotopic samples such as tritium.

a . Heat Fuel Measurements. The calorimeters used for bulk measurements of plutonium or tritium in DOE facilities measure the temperature difference $\Delta T$ generated by a heat source across a thermal resistance. The quantity $\Delta T$ is either measured passively or is actively controlled to within a narrow range. In the passive mode, a sample is inserted in the chamber and the heat flow out of the chamber is determined by measuring a differential voltage $\Delta V$ developed by a constant current through temperature-sensitive resistance sensors. The differential voltage is directly proportional to the difference $\Delta T$ between the temperature of the heat sensor located adjacent to the sample chamber and an identical reference sensor in contact with a constant-temperature air or water bath. The water bath temperature is typically controlled to $0.001^{\circ} \mathrm{C}$. The $\Delta V$ between the sample and the reference is continually measured until an actual or predicted equilibrium is reached. The measurement time at which $\Delta T$ is within $0.1 \%$ equilibrium ranges from 4 to $10 \mathrm{~h}$ depending on the sample matrix and whether the sample temperature is preconditioned.

In the case of active temperature control, an internal wire-wound electrical heater built in the calorimeter between the sample sensor and sample chamber is used. The heat generated by the heater simulates the heat generated from a sample of SNM or tritium. The heater is used to maintain a constant sample-side power $W$ and hence a constant $\Delta T$ between the sample and reference sensors even though there might be no sample in the calorimeter. Addition of a heat-generating radioactive source in the sample chamber initially increases $\Delta T$ beyond a temperature-controller set point. As a result, the power to the heater coil in the sample side is automatically reduced until the preset $\Delta T$ is reached. The magnitude of the power drop to the heater is equivalent to the sample power. This active mode of operation is called servo-controlled. In the servo mode, equilibrium is reached in 2 to $6 \mathrm{~h}$. 
The procedure for calibrating a calorimeter is similar in principle to some other NDA techniques. The signal $\Delta V$, corresponding to a $\Delta T$, is related to the power $W$ by a constant of proportionality. The calibration constant $K$, the calorimetry sensitivity, is determined by the use of calibration heaters or radioactive heat standards using Eq. 7.

$$
K=\Delta V_{\text {std }} / W_{\text {std }}
$$

The sensitivity depends on the design of the calorimeter, in particular the thermal resistance between the sample sensor and the reference sensor. The greater the thermal resistance the more sensitive the calorimeter. Higher sensitivity calorimeters require more time to reach equilibrium. Typical calorimetry sensitivities range from 4000 to 100000 $\mu \mathrm{V} / \mathrm{W}$. There is a small dependence of $K$ on the sample power. In one case, a $1.6 \%$ decrease in sensitivity was observed for standard powers increasing from 0.1 to $10 \mathrm{~W}$. For the passive mode of calorirneter operation, the sensitivity $K$ calculated from Eq. 7 is used with the $\Delta V$ measurement of an unknown to determine its thermal power. In the servo-controlled mode of calorimeter, operation $K$ is not used to measure the power of a sample.

b . Specific Power and SNM Mass Determination. ${ }^{1}$ For a single radioisotope, the power is related to the quantity of material by Eq. 8 .

$$
W=2119.3 Q M / T_{1 / 2} A,
$$

where

$$
\begin{aligned}
& W=\text { power, in watts, emitted by the unknown; } \\
& Q=\text { total disintegration energy, in } \mathrm{MeV} \text {, of alpha particle emitters } \\
& \text { or the average energy, in } \mathrm{MeV} \text {, of beta particles; } \\
& M \text { = mass, in grams, of the radionuclide; } \\
& T_{1 / 2}=\text { half-life, in years, of the radionuclide; and } \\
& A=\text { gram atomic weight of the radionuclide. }
\end{aligned}
$$

The equation can be simplified and rearranged to give

$$
M=W / P,
$$

where $P=$ specific power, in $\mathrm{W} / \mathrm{g}$, of the radionuclide. For example, this constant is $0.3240 \mathrm{~W} / \mathrm{g}$ for tritium and $0.0019288 \mathrm{~W} / \mathrm{g}$ for ${ }^{239} \mathrm{Pu}$. When there is a only a single isotope in the sample, Eq. 9 is used for assay.

For a mixture of radionuclides, the sample power is the sum of the powers of the individual radioisotopes

$$
W=\Sigma_{i} W_{i}=\Sigma_{i} M_{i} P_{i}=M \Sigma_{i} R_{i} P_{i}=M P_{\mathrm{eff}}
$$


where $M$ is the mass of the element being assayed, $R_{i}$ is the isotopic abundance for component $i$ and other power-generating isotopes relative to the element mass, and $P_{\text {eff }}$ is the specific power of the sample for multi-isotopic samples. In this case, the assay equation is

$$
M=W / P_{\text {eff }}
$$

The isotopic abundances of containers of plutonium-bearing materials are typically measured by high-resolution gamma-ray spectroscopy using germanium detectors. Mass spectroscopy and alpha spectroscopy are used to determine the isotopic abundance of plutonium used for ${ }^{238} \mathrm{Pu}$ heat standards.

2. Standard Requirements. Calorimetric measurements require a heat source that produces a known amount of thermal power. This can be produced by (1) a radioactive heat source standard, or (2) an electrical standard that generates thermal power by resistance heating. Both types of standards can be used to determine the calibration constant $K$ in Eq. 7. The only criteria for the standards are that the range of standard heats used to initially calibrate the calorimeter be larger than the expected range of unknown heats and that the upper thermal power is within the design criterion of the calorimeter. For certain calorimeters internal temperatures greater than $40^{\circ} \mathrm{C}$ may cause epoxy components to lose structural integrity. Standards requirements for gamma-ray isotopic measurements are described elsewhere in this guide.

a. Radioactive Heat Standards. Materials that can generate quantities of heat similar in magnitude to the items being measured for long periods of time are suitable as heat standards. Radioactive materials that decay primarily by alpha or beta decay with little or no gamma-ray decay are primary candidates for this application. The short range of the alpha and beta particles ensures that all the energy is deposited in the source material or encapsulation and is converted to heat. The low intensity of penetrating gamma rays also reduces the radiation exposure for personnel using the standards. Plutonium-238 meets these requirements and is the only radioisotope used for radioactive heat standards. A typical isotopic composition of a ${ }^{238} \mathrm{Pu}$ heat standard is shown in Table IX. The thermal power and decay rate of the heat standard are dominated by the $80 \%{ }^{238} \mathrm{Pu}$ component. The chemical composition of the heat source is $\mathrm{PuO}_{2}$. The isotopic composition of the heat source must be determined with techniques using reference materials traceable to NIST.

Table IX

Isotopic Composition of ${ }^{238} \mathrm{Pu}$ Heat Standard

\begin{tabular}{|c|c|}
\hline Isotope & Weight Fraction \\
\hline${ }^{238} \mathrm{Pu}$ & 0.795 \\
\hline${ }^{239} \mathrm{Pu}$ & 0.166 \\
\hline${ }^{240} \mathrm{Pu}$ & 0.029 \\
\hline${ }^{241} \mathrm{Pu}$ & 0.007 \\
\hline${ }^{242} \mathrm{Pu}$ & 0.003 \\
\hline${ }^{236} \mathrm{Pu}$ & $5.6 \times 10^{-7}$ \\
\hline
\end{tabular}


The physical dimensions of the encapsulated ${ }^{238} \mathrm{Pu}$ sources are typically small in comparison to the containers that are being measured. Plutonium-238 standard sizes range from the size of a pencil eraser for the lowest power to a 0.5-1 volume for the largest. These heat sources are relatively small because the high specific power of ${ }^{238} \mathrm{Pu}, 567$ $\mathrm{mW} / \mathrm{g}$, which is about 300 times greater than that of ${ }^{239} \mathrm{Pu}$, leads to a relative reduction by a factor of 300 in standard volume relative to ${ }^{239} \mathrm{Pu}$. Plutonium- 238 heat sources have been constructed with power outputs ranging from 0.0008 to $115 \mathrm{~W}$. Higher wattage sources require storage in a heat sink such as a metal block, metal-shot-filled container, or water bath. The radioactive material is encapsulated in several layers of metal rugged enough to withstand high temperatures and severe mechanical shock. A schematic drawing showing the multilayer containerization of a heat source is shown in Fig. 15. Plutonium-238 heat standards having a thermal power of $1 \mathrm{~W}$ have dose rates ranging from $0.2-0.4 \mathrm{mrem} / \mathrm{h}$ for neutrons and $0.8-1.0 \mathrm{mrem} / \mathrm{h}$ for gammas at $15 \mathrm{~cm}$. The neutron emission is due to $(\alpha, \mathrm{n})$ reactions on the ${ }^{17} \mathrm{O}$ and ${ }^{18} \mathrm{O}$ of the oxide component of the standard. If the $\mathrm{PuO}_{2}$ in the source is prepared with oxygen enriched in ${ }^{16} \mathrm{O}$, the neutron dose rate will be reduced.

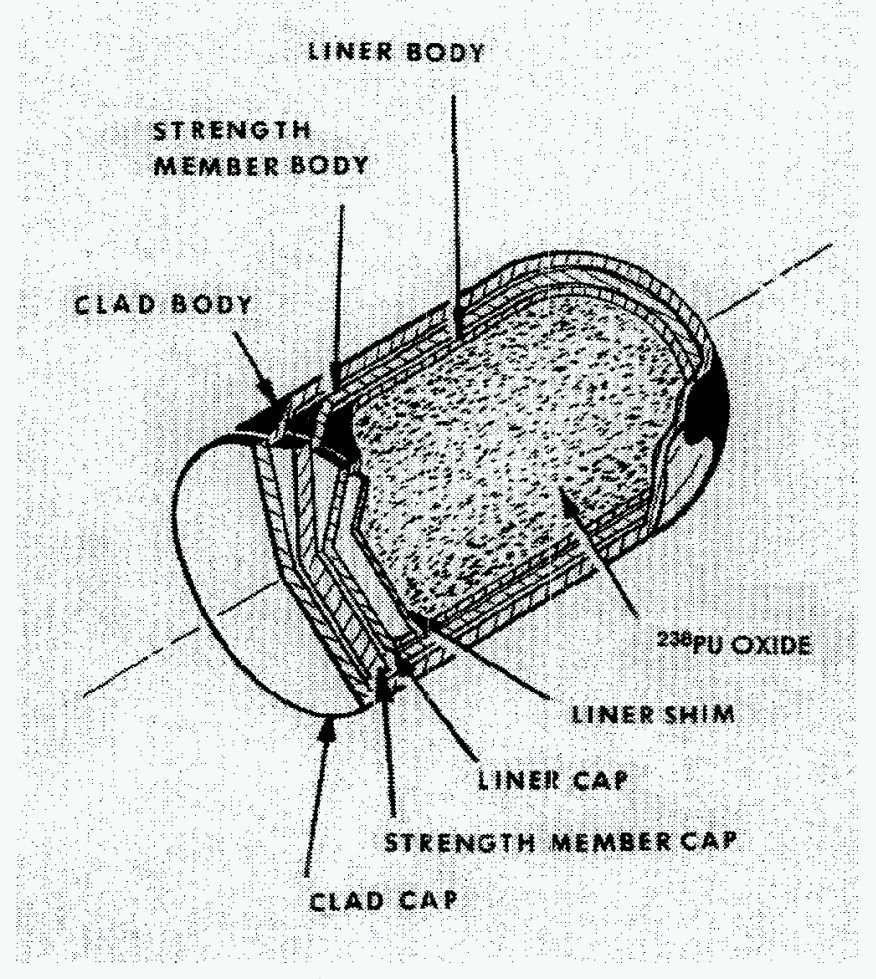

Fig. 15. Plutonium-238 he at standard.

The power of a ${ }^{238} \mathrm{Pu}$ heat source is established by measurement in a calibration calorimeter that uses voltmeters and resistors traceable to NIST electrical standards. These calorimeters have a built-in resistance heater surrounding the sample chamber. The resistance heater is in series with a calibrated standard resistance. The unknown heat output of the ${ }^{238} \mathrm{Pu}$ source is measured with the net sensor output $\Delta V_{u}$, at equilibrium. The sample is then removed from the chamber. Current is applied to the calibration heater so that the power generated is approximately that of the new source with new sensor output $\Delta V_{s}$. The power from the resistance heater $W_{k}$ is $E_{h} I_{\mathrm{h}}$, where $E_{h}$, is the voltage across the 
resistance heater measured by a calibrated digital voltmeter (DVM) and $I_{h}$ is the current. The current is calculated from $I_{h}=E_{s} / R_{s}$, where the voltage $E_{s}$ is measured by the calibrated digital voltmeter across the standard resistance $R_{s}$. The unknown heat standard power $W_{v}$ is calculated from

$$
W_{u}=W_{k}+\left(\Delta V_{u}-\Delta V_{k}\right) / K_{k}
$$

where $K_{k}$ is the sensitivity of the calorimeter at the power level $W_{k}{ }^{1}$. The DVM used for the measurements of $E_{h}, E_{s}, \Delta V_{u}, \Delta V_{k}$, and $K_{k}$ and the standard resistance $R_{s}$ are calibrated by the site electrical calibration laboratory. There the voltmeter is checked by measurement of a standard voltage and the resistance $R_{s}$ is compared to standard resistors maintained by the electrical calibration laboratory. In turn the calibration laboratory standards are traceable to NIST through the Albuquerque Operations Primary Standards Laboratory, Sandia National Laboratory, Albuquerque, New Mexico. Typically the voltmeter is certified to be accurate to $10 \mathrm{ppm}$ and the standard resistance to $15 \mathrm{ppm}$. The accuracy of the ${ }^{238} \mathrm{Pu}$ heat standard must take into account the heat distribution error of the standards calorimeter and the uncertainty in the decayed thermal power due to isotopic uncertainties. The overall accuracy of a heat standard calibrated at Mound Applied Technologies for a 3 to 5 year calibration time period is typically $150 \mathrm{ppm}(=0.015 \%)$. A calibration certificate that was issued for a ${ }^{238} \mathrm{Pu}$ standard calibrated at Mound is shown in Fig. 16. Larger heat sources that cannot fit within the sample chamber of the calibration

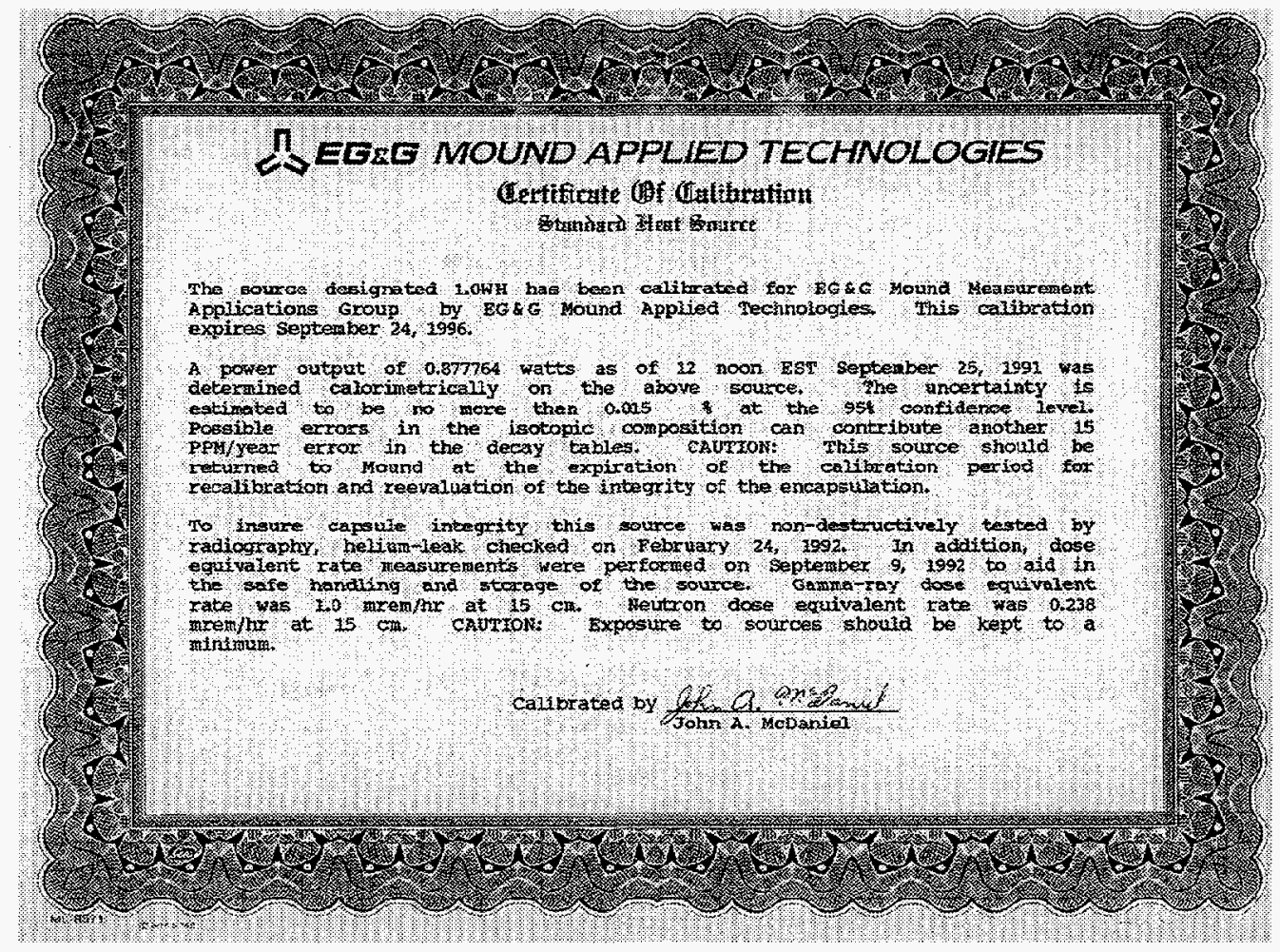

Fig. 16. Plutonium-238 heat source calibration certificate. 
calorimeter can be calibrated by comparison to sums of known smaller sources in another calorimeter. The overall uncertainty for these higher power sources is larger than for the smaller sources. For example, for a $115-\mathrm{W}$ heat source the calibration accuracy was $0.075 \%$.

b. Electrical Standards. Electrical standards can be used for calorimeter calibration. With this approach portable heater probes are inserted into an adapter sleeve inside the sample chamber and connected to a constant current source and a DVM. The methodology for use of the probe is similar to the methodology for primary calibration with an electrical heater inside a calibration calorimeter. The amount of power $W$ generated in the heater is $W=E I$, where $E$ is the potential difference across the heater and $I$ is the current flowing through the heater. The quantity $E$ is measured with a high-precision calibrated voltmeter and $I$ is determined by a separate voltage measurement across a standard resistor placed in series with the resistance heater. Calibration with a heater probe has a more direct traceability link to NIST than calibration with ${ }^{238} \mathrm{Pu}$ standards.

There are certain advantages to using electrical standards compared to ${ }^{238} \mathrm{Pu}$ heat sources. The ${ }^{238} \mathrm{Pu}$ heat sources are accountable nuclear material and must be handled following MC\&A requirements. There are no such requirements with electrical standards. For ${ }^{238} \mathrm{Pu}$ standards, the radioactive decay of the samples leads to a constantly decreasing power. For this reason, the ${ }^{238} \mathrm{Pu}$ standards periodically must be sent to another site for recalibration. Shipping requirements add to the expense and delay involved in off-site calibrations. Electrical standards must also be recalibrated, but this can usually be done onsite, with no shipping requirements and for a much smaller cost. The integrity of ${ }^{238} \mathrm{Pu}$ containers must be periodically tested by nondestructive testing (NDT) methods. This is not a requirement for the electrical standards. A disadvantage to using an electrical standard is the relatively complicated procedure for using the heater probe gear. No such complication arises with ${ }^{238} \mathrm{Pu}$ heat sources. Another relative disadvantage with the portable electrical standard is that the DVM and standard resistor must be recertified 3 to 20 times more frequently than for the ${ }^{238} \mathrm{Pu}$ sources. The certification period for ${ }^{238} \mathrm{Pu}$ heat sources is longer (3-5 years) than the certification period for the electrical standards (312 months). If the electrical equipment has shown a history of operating satisfactorily, the frequency of the recalibration will be reduced by the electrical calibration laboratory.

Both ${ }^{238} \mathrm{Pu}$ and the electrical standard will not be available for use during recalibration. Traceability to NIST is not maintained if measurements are performed with equipment whose certification period has expired. Additional calibrated ${ }^{238} \mathrm{Pu}$ heat standards or DVMs and standards resistors must be available to replace the units being calibrated to ensure continuity of measurement control in a production environment. Calibration tags indicating the expiration date of the certification of the electrical equipment should be affixed to the front panel of the equipment. A separate certificate specifying the accuracy of the component for the certification period should be maintained. No calibration tags are used with ${ }^{238} \mathrm{Pu}$ heat sources.

Calibration heater probes have been produced by Mound for use in calorimeters. The Mound Electrical Calibration Heater $(\mathrm{MECH})$ probe has been used at Los Alamos for accountability measurements. ${ }^{2} \mathrm{~A}$ schematic of the MECH probe is given in Fig. 17. The heater element is located at the tip of the probe. The thermal power is supplied to 


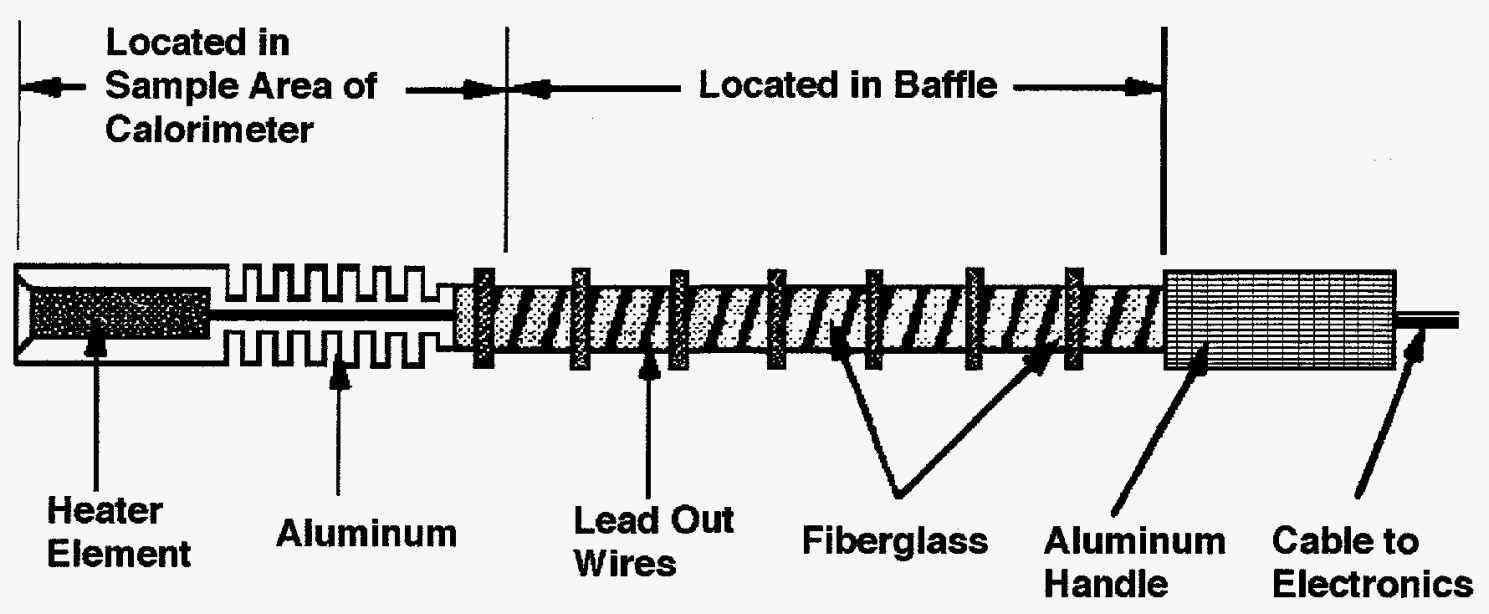

Fig. 17. MECH probe.

the probe through lead wires from an external power supply. Different sizes of probes have been constructed to fit a range of container sizes with thermal power outputs ranging from $0.1 \mathrm{~W}$ to $150 \mathrm{~W}$. A scanning DVM alternately measures the voltages across the heater resistor and the standard resistor in series to determine the heater power. Both the DVM and standard resistor are easily removable for periodic calibration. The power delivered to the probe is measured and controlled by computer. Specially constructed calorimeter cans, adapter sleeves, and baffles need to be constructed to allow insertion of the heater probe. A photograph of the components is shown in Fig. 18.

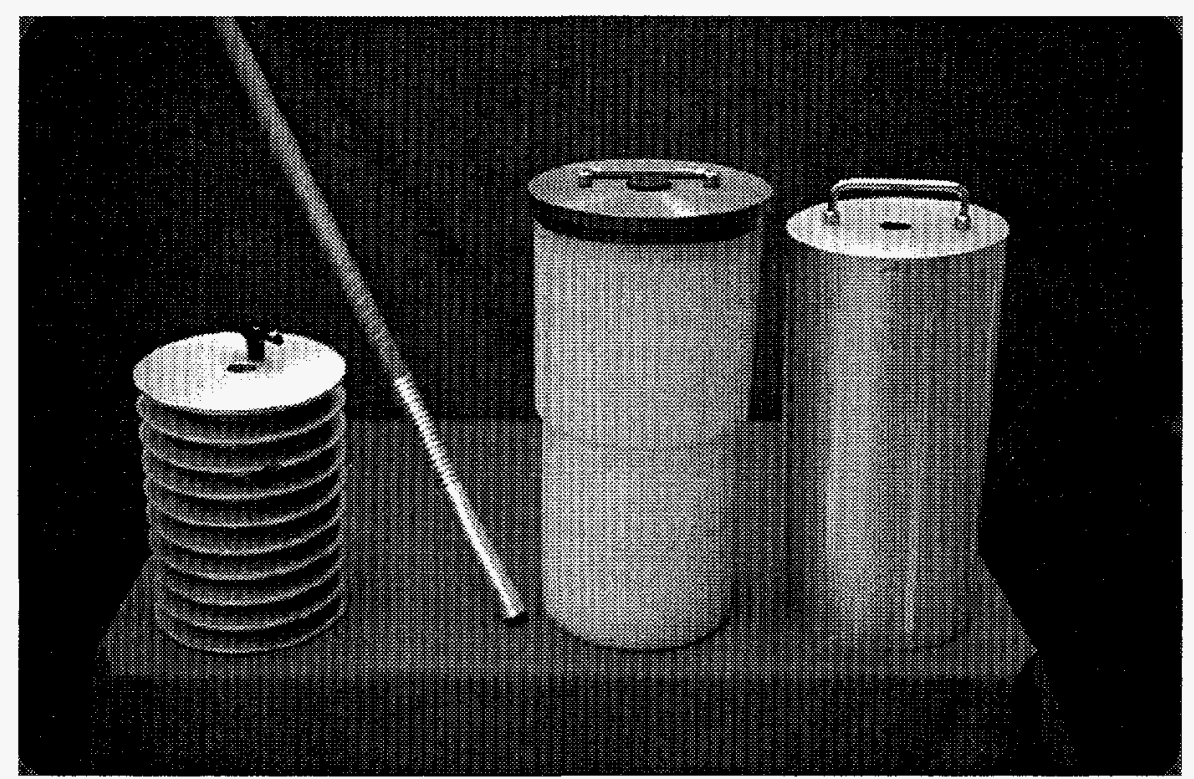

Fig. 18. MECH probe components. From left to right, aluminum adapter sleeve, electrical probe, insulated baffle, and calorimeter can. 
A small part of the measured thermal power delivered into the calorimeter is dissipated in the electrical leads and not measured by the heat sensors. Thus the actual power delivered to the calorimeter is less than indicated. The bias becomes larger for smaller powers when the resistance of the heater becomes smaller relative to the heater leads. For a $\mathrm{MECH}$ probe operated in the 50 - to $155-\mathrm{W}$ range, the lead loss was estimated to be $0.12 \%$.

The accuracy of the MECH probe has been tested against ${ }^{238} \mathrm{Pu}$ heat sources. Good agreement has been obtained for high-power sources. Agreement of $0.1 \%$ was obtained for measurements at $89 \mathrm{~W}$ and $155 \mathrm{~W}$ for two calorimeters. ${ }^{3}$ The relative bias between the methods becomes larger for lower thermal powers. Relative biases ranging from $0.5 \%$ to $0.8 \%$ were observed for a thermal power range from 1.7 to $7.5 \mathrm{~W}$ for one calorimeter. ${ }^{2}$

\section{References}

1. "Plutonium-Bearing Solids- Calibration Techniques for Calorimetric Assay," ANSI N15.22-1987 (American National Standards Institute, Inc., New York, 1987).

2. D. P. Renz, J. A. McDaniels, and J. R. Wetzel, "Test Results of the MECH Probe at Mound," Nucl. Mater. Mange. XXII (Proc. Issue), 1130-1134 (1993).

3. D. P. Renz, J. R. Wetzel, M. B. Severinghaus, R. I. Sheldon, and L. Bruckner, "High Wattage Comparison of the MECH Probe and ${ }^{238} \mathrm{Pu}$ Heat Standards at Los Alamos," Nucl. Mater. Manage. XXIII (Proc. Issue), 95-99 (1994). 


\section{CURRENTLY AVAILABLE NONDESTRUCTIVE ASSAY STAN- DARDS}

In this chapter we will discuss NDA standards that are currently available from US or European laboratories. Only two CRM standards have been prepared expressly for NDA of SNM and we will discuss them in some detail. To supplement this limited repertoire of CRM standards, scientists throughout the US DOE complex have fabricated many different types of WRM standards and we will discuss a few of them to illustrate the principles of the design and fabrication of NDA standards.

\section{A. Certified Reference Materials}

Only two available sets of CRM standards have been prepared expressly for the calibration of NDA techniques. Both sets are intended for isotopic measurements: one for plutonium and one for uranium. There are several reasons that only two sets of CRM standards exist after 30 years of safeguards activities. Each set of CRM standards took more than 10 years to prepare from the initial planning, through the preparation and characterization of the SNM, to the final completion and delivery. CRM standards must be shipped from the producing laboratory to the user, and it is well known that the transportation of SNM across a country and especially across the border of a country is heavily regulated and therefore nontrivial. An international purchase of a CRM standard must first be approved by the SNM regulatory authorities in both countries, and then the packaging of the SNM must conform to the shipping requirements of both countries. The fact that the purchase of CBNM CRM 271 from Europe by a US laboratory took approximately four years of time and effort is a vivid illustration of the difficulties involved.

Both of these sets of CRM standards contain relatively small amounts of SNM, whereas the amount of SNM required for a proper verification measurement with some of the NDA techniques, such as neutron counting and waste measurement, may exceed several kilograms of SNM. This requirement for standards containing substantial quantities of SNM creates very stringent practical and regulatory difficulties. In many such situations, the only viable alternative is to prepare and characterize the necessary SNM as a WRM at the laboratory where it is needed.

1. CBNM Nuclear Reference Material 271. This CRM standard set is intended to be used for the nondestructive determination of the isotopic distribution of ${ }^{238} \mathrm{Pu},{ }^{239} \mathrm{Pu},{ }^{240} \mathrm{Pu},{ }^{241} \mathrm{Pu}$, and ${ }^{241} \mathrm{Am}$ by gamma-ray spectrometry. This standard set was prepared by the CBNM, now called the IRMM in Geel, Belgium, and it has a certification date of 20 June 1986. The set consists of four sealed stainless steel containers, each containing a sintered pellet of about $6.6 \mathrm{~g}$ of $\mathrm{PuO}_{2}$. The cans are $40 \mathrm{~mm}$ in diameter and 21 $\mathrm{mm}$ tall, each containing one of four isotopic compositions of plutonium.

The cans are labeled according to their nominal ${ }^{239} \mathrm{Pu}$ percentage as CBNM Pu 93, CBNM Pu 84, CBNM Pu 70, and CBNM Pu 61. They are certified for ${ }^{238} \mathrm{Pu}^{242} \mathrm{Pu}$ and

${ }^{241} \mathrm{Am}$ isotopic fractions and the certified isotopic distribution as of June 20,1986 , is 
shown in Table X. Only a few sets of this CRM standard exist in the United States (Los Alamos, LLNL, and NBL). The user should directly contact IRMM for availability.

A picture of the CBNM 271 set is shown in Fig. 19. and the schematic of the CRM is shown in Fig. 20.

Table X

Plutonium Isotopic Abundance on

June 20, 1986

Atom Percent

\begin{tabular}{|l|l|l|l|l|l|l|}
\hline Material & ${ }^{238} \mathrm{Pu}$ & ${ }^{239} \mathrm{Pu}$ & ${ }^{240} \mathrm{Pu}$ & ${ }^{241} \mathrm{Pu}$ & ${ }^{242} \mathrm{Pu}$ & ${ }^{241} \mathrm{Am} / \mathrm{Pu}$ \\
\hline CBNM Pu 93 & 0.0117 & 93.4392 & 6.2886 & 0.2215 & 0.0390 & 0.1039 \\
\hline CBNM Pu 84 & 0.0706 & 84.3985 & 14.1578 & 1.0197 & 0.3534 & 0.2157 \\
\hline CBNM Pu 70 & 0.8506 & 73.4248 & 18.2445 & 5.4257 & 2.0544 & 1.1624 \\
\hline CBNM Pu 61 & 1.2045 & 62.6562 & 25.3526 & 6.6376 & 4.1491 & 1.4362 \\
\hline
\end{tabular}

\begin{tabular}{|l|l|l|l|l|l|l|}
\hline Material & ${ }^{238} \mathrm{Pu}$ & ${ }^{239} \mathrm{Pu}$ & ${ }^{240} \mathrm{Pu}$ & ${ }^{241} \mathrm{Pu}$ & ${ }^{242} \mathrm{Pu}$ & ${ }^{241} \mathrm{Am} / \mathrm{Pu}$ \\
\hline CBNM Pu 93 & 0.0117 & 93.4123 & 6.3131 & 0.2235 & 0.0395 & 0.1047 \\
\hline CBNM Pu 84 & 0.0703 & 84.3377 & 14.2069 & 1.0275 & 0.3576 & 0.2173 \\
\hline CBNM Pu 70 & 0.8458 & 73.3191 & 18.2945 & 5.4634 & 2.0772 & 1.1705 \\
\hline CBNM Pu 61 & 1.1969 & 62.5255 & 25.4058 & 6.6793 & 4.1925 & 1.4452 \\
\hline
\end{tabular}

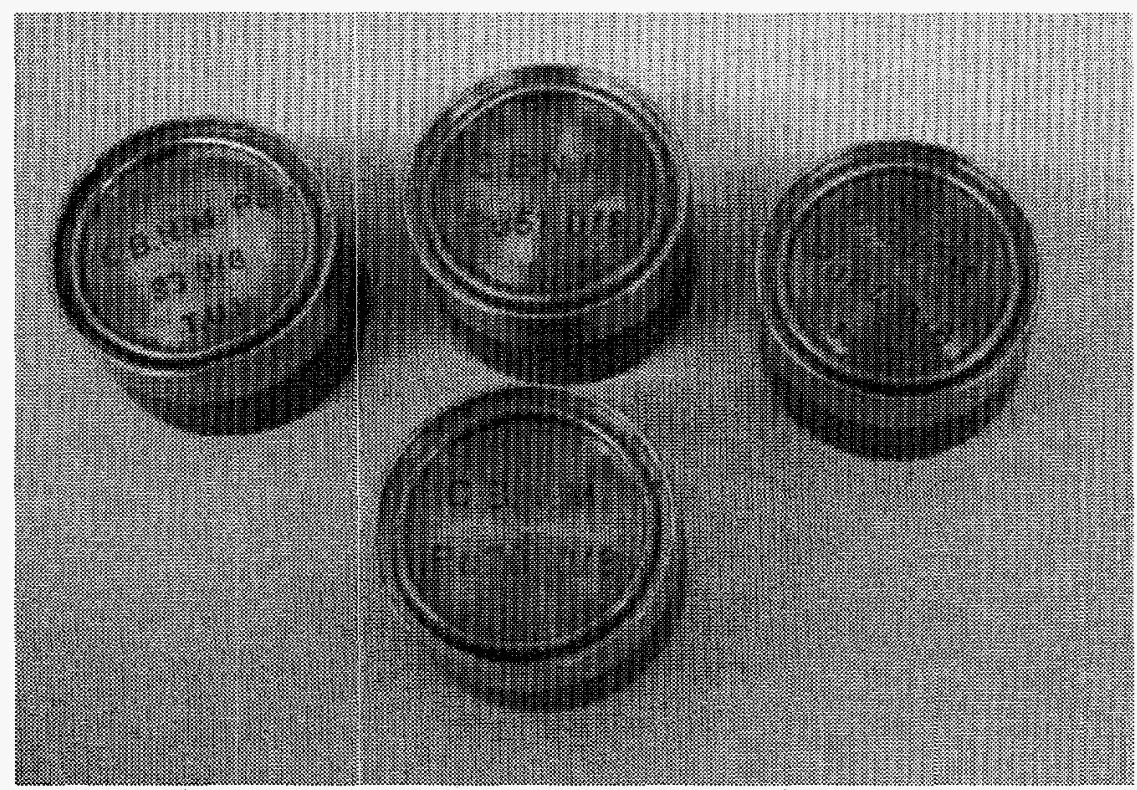

Fig. 19. Picture of CBNM 271 plutonium isotopic CRM. 


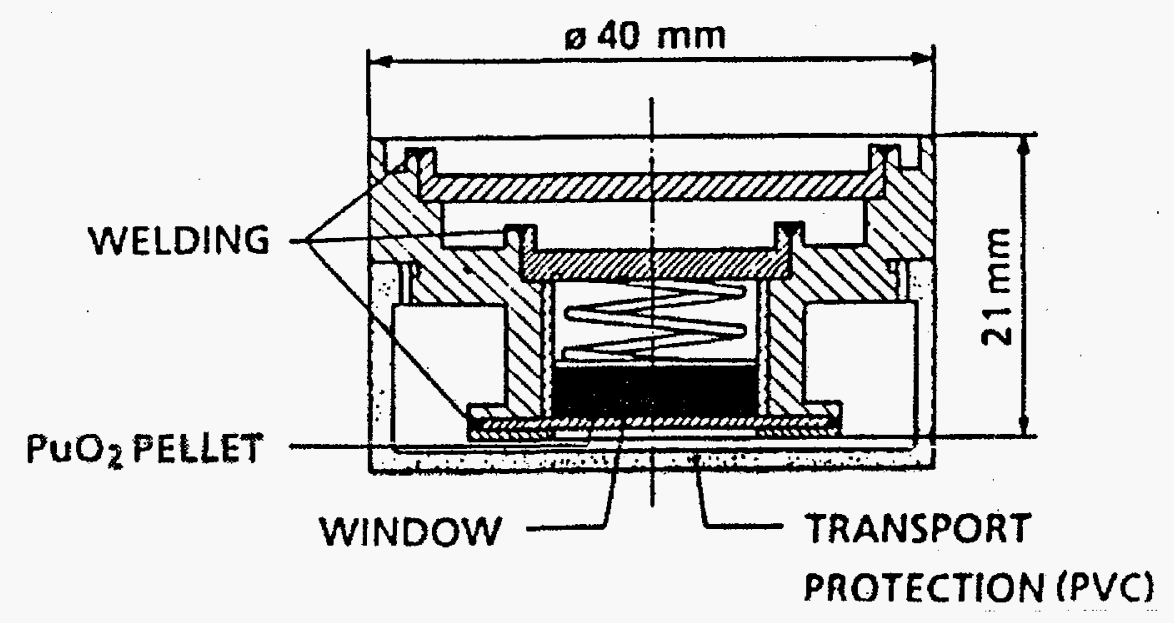

Fig. 20. Schematic drawing of CBNM 271.

\section{Certified Nuclear Reference Material EC NRM 171/NBL CRM}

969. This low-enriched uranium CRM standard was prepared primarily for uranium enrichment measurements, and it was certified with an accuracy of better than $\pm 0.1 \%$. This CRM was issued in 1985 and it was prepared and certified jointly by CBNM and NBL, representing the first example of an internationally certified reference material for NDA. ${ }^{1}$ This CRM may be obtained from the NBL in Argonne, Illinois. P. Matussek of Kernforschungzentrum Karlsruhe $(\mathrm{KfK})^{2}$ wrote a very good user's manual for this CRM, and it provides a comprehensive description of the enrichment principle and the factors that one needs to consider during a uranium enrichment measurement.

This CRM standard represents a physical standard in the sense that it provides wellcharacterized bulk quantities of uranium in a well-defined, invariable geometry. The CRM consists of $\mathrm{U}_{3} \mathrm{O}_{8}$ powder with five different ${ }^{235} \mathrm{U}$ enrichments: $0.3 \%, 0.7 \%, 1.9 \%, 2.9 \%$, and $4.5 \%$. The range of enrichment was chosen to span the range of ${ }^{235} \mathrm{U}$ enrichments that are commonly encountered in the low-enrichment uranium fuel cycle. Each sample consists of $200 \mathrm{~g}$ of $\mathrm{U}_{3} \mathrm{O}_{8}$ powder sealed in a cylindrical aluminum with an inner diameter of $70 \mathrm{~mm}$. The 70-mm diameter and $200 \mathrm{~g}$ represent the minimum size of sample that is required to produce $99.9 \%$ of the gamma radiation (at $186 \mathrm{keV}$ ) that would be obtained from an infinitely thick sample. The physical geometry of the standard is shown in Fig. 21 and a picture of the containers composing this CRM standard is shown in Fig. 22.

The enrichment of this set of CRM standards is certified as listed in Table XI.

\section{B . Working Reference Material}

WRM is usually prepared by a single laboratory for its own use as a calibration standard, as a control standard, or for the qualification of an NDA measurement method. As such it may not be necessary to ship it from site to site, thus eliminating many regulatory difficulties. Because many sets of WRM standards have been fabricated by different facilities throughout the years, the discussion here cannot be complete. Rather we have selected several well-characterized sets to illustrate the principles of preparing WRMs. 


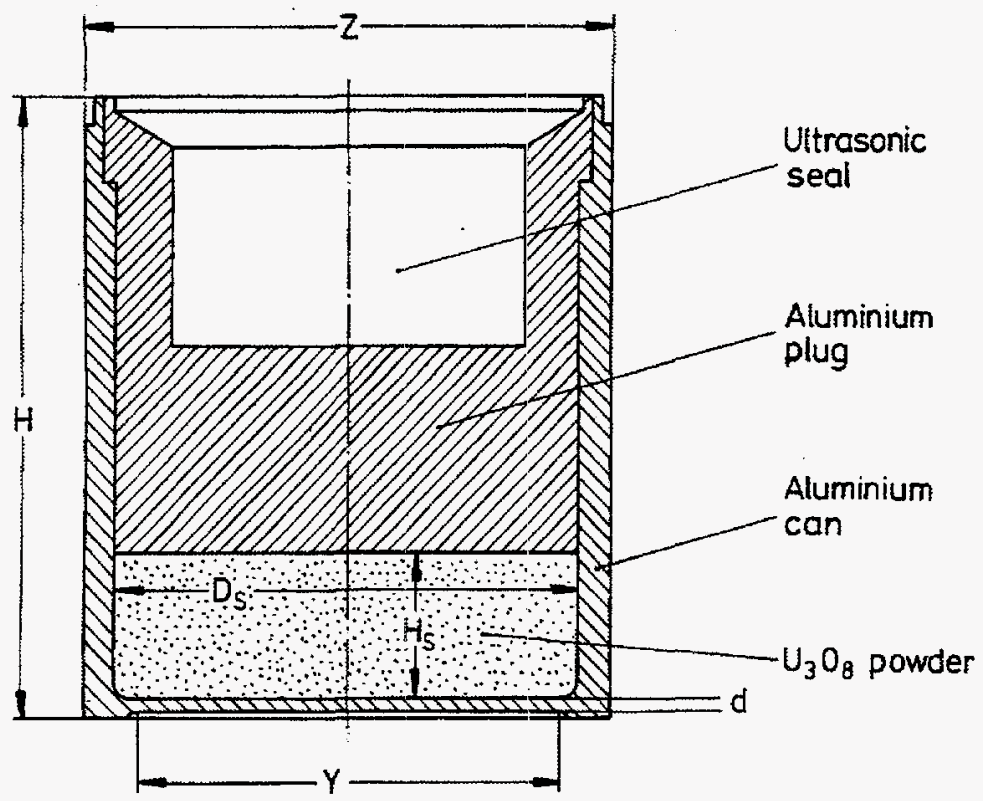

Fig. 21. Cross-sectional view of EC-NRM-171/ NBL-CRM-969 standard.

Sample dimensions:

Total height

Outer diameter

Inner diameter

Window diameter

Window thickness

$\mathrm{U}_{3} \mathrm{O}_{8}$ material height
$\mathrm{H}=90.0 \mathrm{~mm}$

$\mathrm{Z}=80.0 \mathrm{~mm}$

$\mathrm{D}_{\mathrm{s}}=70.0 \mathrm{~mm}$

$\mathrm{Y}=66.0 \mathrm{~mm}$

$\mathrm{d}=2.0 \mathrm{~mm}$

$\mathrm{H}_{\mathrm{s}}=20.8 \mathrm{~mm}$

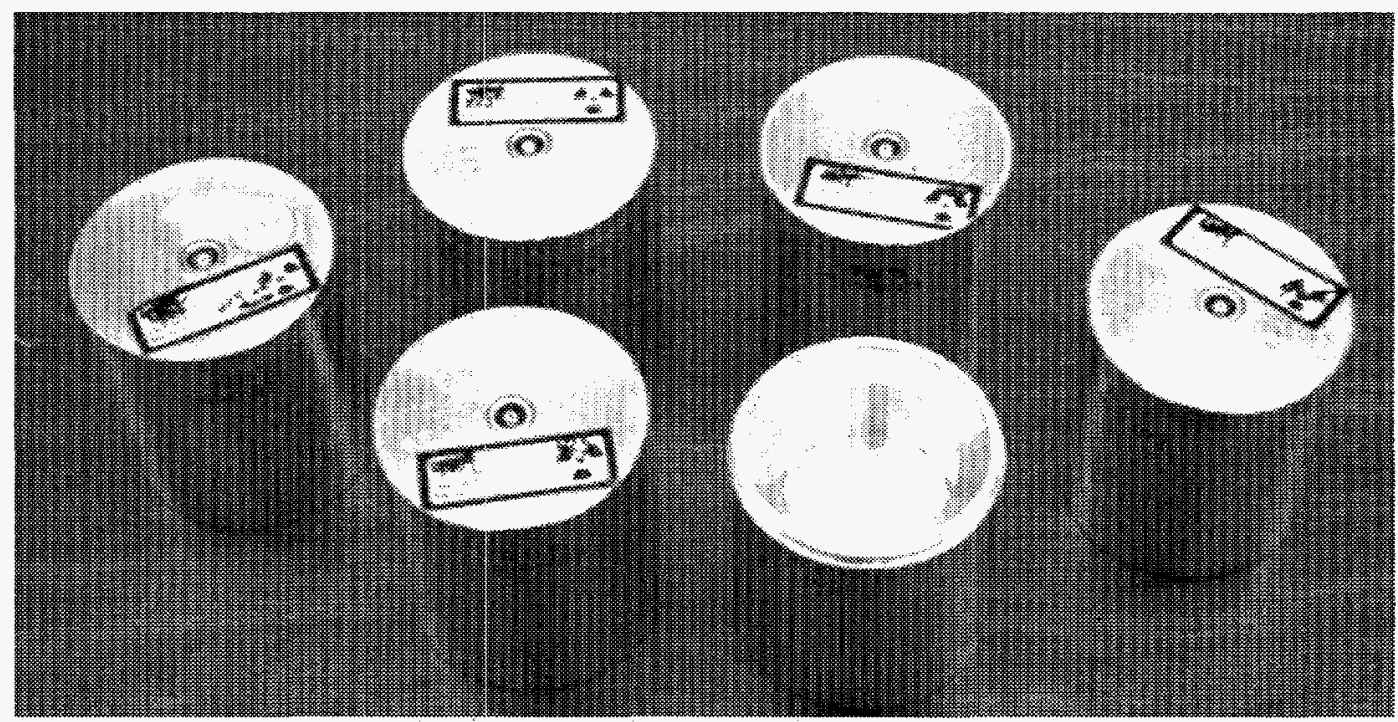

Fig. 22. Picture of the EC-NRM-171/NBL-CRM-969 standards set. The can at the bottom of the picture is an empty container that is identical to the actual containers. 
Table XI

Enrichment of EC-NRM-171/NBL-CRM-969 Standard

\begin{tabular}{|c|c|c|c|c|}
\hline \multicolumn{1}{|c|}{ Code } & Material & Certified Value & Unit & $\begin{array}{c}\text { Price } \\
\text { (ECU) }\end{array}$ \\
\hline \multirow{3}{*}{ EC-NRM 171 } & Set of $5 \mathrm{Al}$ cans with $\mathrm{U}_{3} \mathrm{O}_{8}$ of & $0.7209 \pm 0.0005$ at $\%{ }^{235} \mathrm{U}$ & $5 \mathrm{X} 200 \mathrm{~g}$ & 7260 \\
& different ${ }^{235} \mathrm{U} / \mathrm{U}$ abundances & $1.9664 \pm 0.0014$ at $\%{ }^{235} \mathrm{U}$ & & \\
& & $2.9857 \pm 0.0021$ at $\%{ }^{235} \mathrm{U}$ & & \\
& & $4.5168 \pm 0.0032$ at $\%{ }^{235} \mathrm{U}$ & & \\
& & & & \\
& & & & \\
\end{tabular}

1. Performance Laboratory Standards. In Europe, the EURATOM laboratory at Ispra, Italy, has the most extensive collection of WRM standards. At Ispra, PERLA was established over twenty years ago with the main goal of providing a wide variety of uranium and plutonium WRMs to be used mainly for the calibration of NDA instruments and techniques. These WRM standards are very well prepared and characterized by several European facilities and analytical laboratories. The PERLA facility and the available WRM standards can be used by NDA instrument developers to calibrate their instruments and test their techniques. The PERLA facility has also been used to train EURATOM and International Atomic Energy Agency (IAEA) inspectors in making measurements with NDA equipment and for Physical Inventory Verification exercises.

a. Plutonium-Bearing WRM. A set of well-characterized plutonium WRM standards was fabricated in the late 1980s with the main purpose of calibrating neutron coincidence counting (NCC) techniques, although the set can also be used for calorimetry and plutonium isotopic measurements. The quality control of the preparation and characterization of this set of WRMs has been reported. ${ }^{3,4}$ The set consists of high-purity plutonium oxide in sizes ranging from $0.5 \mathrm{~g}$ to $2.5 \mathrm{~kg}$, composed of low-, medium- and high-burnup plutonium (Table XII). Some were packaged in special containers for neutron multiplication studies (MS/1000-S, MS/2500-S) (Table XIII). This set of WRMs is probably the best-characterized low-impurity plutonium oxide standard available for NCC and provides a good example of how WRM should be prepared and characterized.

Table XII

Description of the $\mathrm{PuO}_{2}$ Sample Batches

\begin{tabular}{|l|l|l|l|}
\hline Material & Lot & Description & ${ }^{239} \mathrm{Pu}(\%)$ \\
\hline $\mathrm{PuO}_{2}$ & 1 & Low burnup & $\sim 71$ \\
\hline & 2 & Medium burnup & $\sim 61$ \\
\hline & 3 & High burnup & $\sim 58$ \\
\hline
\end{tabular}


Table XIII

Weight Range and Container

\begin{tabular}{|l|l|l|}
\hline Material & Weight range (g) & Container $^{3}$ \\
\hline $\mathrm{PuO}_{2}$ powder & $0.5-1.0$ & 5 \\
\hline & $50-100$ & 200 \\
\hline & $300-1000$ & 1000 \\
\hline & $1500-2500$ & 2500 \\
\hline & $50-250$ & MS $/ 1000-\mathrm{S}$ \\
\hline & $250-1000$ & MS/2500-S \\
\hline
\end{tabular}

b. MOX WRM. While the above set of plutonium-bearing WRMs was being prepared, a set of MOX powder and pellets was also fabricated (Tables XIV and XV). The MOX pellets were in small rods clad with stainless steel or zircaloy. ${ }^{3}$ Some of the rods were full-size rods representative of fuel rods found in reactors. They comprise a range of $\mathrm{Pu} / \mathrm{U}$ ratios and the plutonium ranges from low-to-high burnup. This set of MOX rod WRM is also very well characterized and it is an excellent standard for calibrating NCC systems.

Table XIV

Description of the MOX Rods

\begin{tabular}{|l|l|l|l|l|l|}
\hline Material & Lot & Description & Pu weight $\%$ & ${ }^{239} \mathrm{Pu} / \mathrm{Pu}(\%)$ & ${ }^{235} \mathrm{U} / \mathrm{U}(\%)$ \\
\hline MOX & 1 & SNR C1 fuel & $\sim 22$ & $\sim 66$ & natural \\
\hline & 2 & SNR C2 fuel & $\sim 31$ & $\sim 57$ & natural \\
\hline & 3 & FDWR fuel & $\sim 9.3$ & $\sim 79$ & natural \\
\hline & 4 & DWR fuel & $\sim 4.2$ & $\sim 84$ & natural \\
\hline & 5 & KNK II fuel & $\sim 22.0$ & $\sim 66$ & $\sim 63$ \\
\hline
\end{tabular}

Table XV

Weight Range and Container of MOX

\begin{tabular}{|l|l|c|}
\hline Material & Weight range $(\mathrm{g})$ & Container $^{3}$ \\
\hline MOX powder & $0.5-1.0$ & 5 \\
\hline
\end{tabular}

2. PIDIE Plutonium Isotopic Standards. In the late 1980s, the ESARDA working group organized a PIDIE. A set of seven plutonium standards of about $0.4 \mathrm{~g}$ of plutonium, each with nominal ${ }^{240} \mathrm{Pu}$ isotopic percentages of $6.0 \%, 10.0 \%, 14.1 \%, 19.7 \%$, $21.1 \%, 23.8 \%$, and $25.5 \%$ were fabricated at the Atomic Energy Research Establishment (AERE) Harwell. Fig. 23 is a photograph of one set. These standards were distributed as unknowns to nine laboratories in Europe and in the US for NDA measurements in late 1988. After the first results were synthesized by eight participants, a preliminary report was written in 1989. Mass spectrometry measurements of small samples of each plutonium batch were carried out in 1989 and 1990 by six other laboratories 


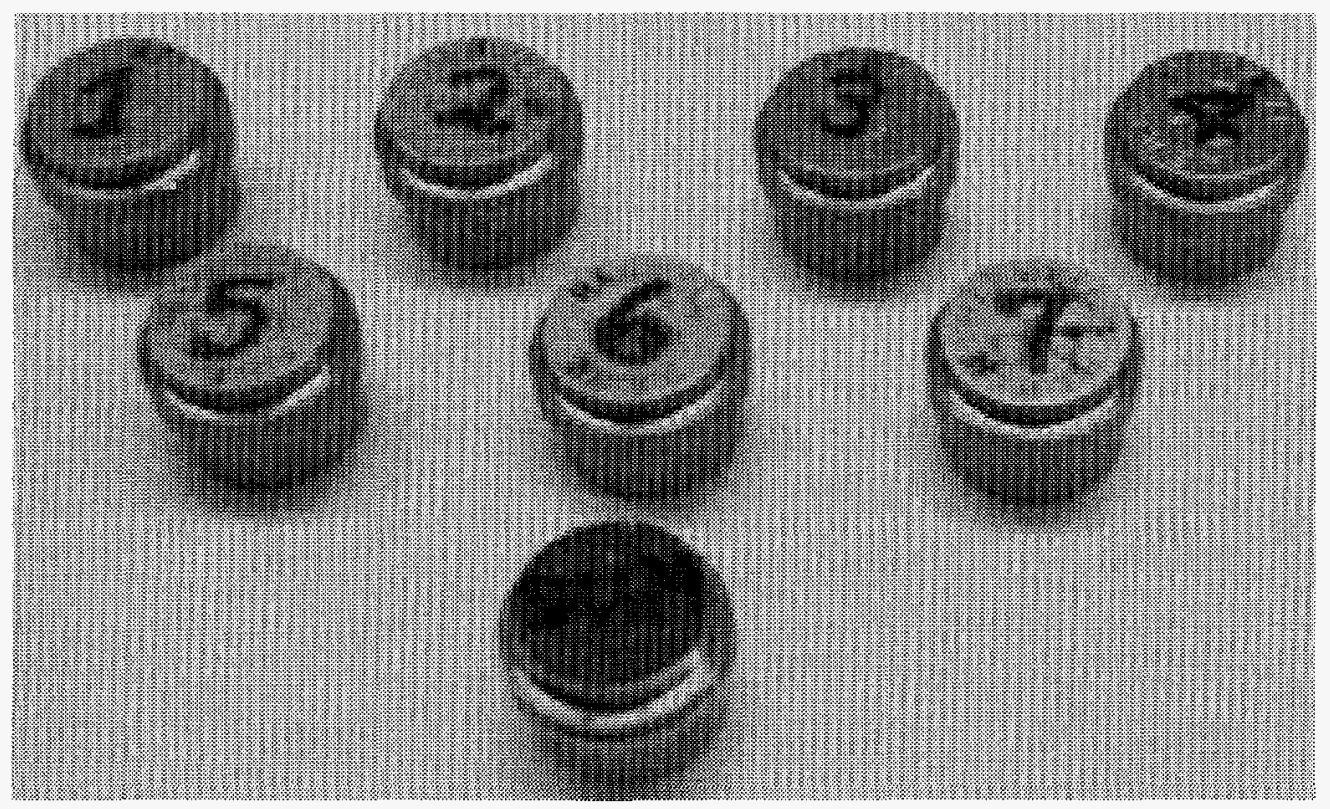

Fig. 23. Picture of the PIDIE plutonium isotopic WRM. The container at the lower part of the picture contains no SNM.

to complete the final analysis of the results. The results of the intercomparison have been reported in Ref. 5. The plutonium isotopic distribution of the PIDIE samples are shown in Table XVI.

3. Los Alamos Fabricated WRM. In the US, Los Alamos has the largest collection of WRM standards, accumulated through thirty years of research and development in NDA. In 1988 a working group was formed at Los Alamos to examine the status of the NDA standards there. ${ }^{6}$ The group concluded that while Los Alamos had many sets of WRM standards, the documentation and the traceability to high-precision DA results was less than desirable. Some of the chemical analysis results were available to only a few staff members. A complete listing of the WRMs existing at that time can be found in the Appendix of Ref. 6. There were also some significant gaps in the availability of WRM standards. Therefore this working group recommended that several new sets of WRM standards should be prepared and that any new standards fabricated at Los Alamos would follow a more rigorous procedure, as outlined in Chapter V. Since 1988 more than ten sets of WRMs have been prepared for a wide range of NDA instruments, and we will now discuss several of the newer WRM standards. These WRMs are well-documented and characterized, and the characterization was as rigorous as that for CRM standards.

4. Segmented Gamma Scanning Plutonium Can Standard. One of the highest priorities for new WRM standards at Los Alamos, as reported by the working group in 1988, was for new plutonium can standards for SGS. Four sets of WRM plutonium can standards, with a total of 20 cans, were fabricated in $1990 .^{7}$ One of these sets was shipped to Westinghouse Savannah River Site (WSRS). 
Table XVI

Plutonium Isotopic Distribution of PIDIE Samples Determined by Los Alamos DA as of January 1, 1988

Atom Percent

\begin{tabular}{|l|l|l|l|l|l|l|}
\hline \multicolumn{1}{|c|}{ Material } & \multicolumn{1}{|c|}{$\begin{array}{c}{ }^{238} \mathrm{Pu} \\
\text { at. } \%\end{array}$} & $\begin{array}{r}{ }^{239} \mathrm{Pu} \\
\text { at. } \%\end{array}$ & \multicolumn{1}{|c|}{${ }^{240} \mathrm{Pu}$} & \multicolumn{1}{c|}{$\begin{array}{c}{ }^{241} \mathrm{Pu} \\
\text { at. \% }\end{array}$} & $\begin{array}{c}{ }^{242} \mathrm{Pu} \\
\text { at. \% }\end{array}$ & \multicolumn{1}{c|}{$\begin{array}{c}{ }^{241} \mathrm{Am} / \mathrm{Pu} \\
\text { at. \% }\end{array}$} \\
\hline PIDIE 1 & 0.0111 & 93.7902 & 5.9669 & 0.1976 & 0.0342 & 0.2257 \\
\hline PIDIE 2 & 0.0227 & 89.3798 & 10.0619 & 0.4424 & 0.0932 & 0.2631 \\
\hline PIDIE 3 & 0.0474 & 84.6386 & 14.0951 & 0.9877 & 0.2311 & 0.6165 \\
\hline PIDIE 4 & 0.1090 & 77.7986 & 19.7053 & 1.8265 & 0.5604 & 1.5541 \\
\hline PIDIE 5 & 0.1321 & 75.9725 & 21.1524 & 2.0490 & 0.6944 & 1.7177 \\
\hline PIDIE 6 & 0.9354 & 66.4662 & 23.8335 & 5.2465 & 3.5186 & 3.7375 \\
\hline PIDIE 7 & 1.2608 & 62.1174 & 25.5418 & 6.4517 & 4.6281 & 3.5492 \\
\hline
\end{tabular}

Mass Percent

\begin{tabular}{|c|c|c|c|c|c|c|}
\hline Material & $\begin{array}{l}{ }^{238} \mathrm{Pu} \\
\text { wt \% }\end{array}$ & $\begin{array}{l}{ }^{239} \mathrm{Pu} \\
\text { wt \% }\end{array}$ & $\begin{array}{l}{ }^{240} \mathrm{Pu} \\
\mathrm{wt} \%\end{array}$ & $\begin{array}{l}{ }^{241} \mathrm{Pu} \\
\text { wt \% }\end{array}$ & $\begin{array}{l}{ }^{242} \mathrm{Pu} \\
\text { wt \% }\end{array}$ & $\begin{array}{c}{ }^{241} \mathrm{Am} / \mathrm{Pu} \\
\text { wt \% }\end{array}$ \\
\hline PIDIE 1 & 0.0111 & 93.7649 & 5.9902 & 0.1992 & 0.0346 & 0.2275 \\
\hline PIDIE 2 & 0.0226 & 89.3379 & 10.0993 & 0.4459 & 0.0943 & 0.2651 \\
\hline PIDIE 3 & 0.0472 & 84.5795 & 14.1442 & 0.9953 & 0.2338 & 0.6212 \\
\hline PIDIE 4 & 0.1084 & 77.7175 & 19.7673 & 1.8399 & 0.5669 & 1.5655 \\
\hline PIDIE 5 & 0.1314 & 75.8857 & 21.2168 & 2.0638 & 0.7023 & 1.7301 \\
\hline PIDIE 6 & 0.9297 & 66.3439 & 23.8893 & 5.2807 & 3.5563 & 3.7619 \\
\hline PIDIE 7 & 1.2528 & 61.9848 & 25.5941 & 6.4919 & 4.6763 & 3.5713 \\
\hline
\end{tabular}

These standards were in stainless steel containers designed specifically for the can SGS. The matrix material used was DE with a density of 0.26 units. The plutonium oxide was high-fired, blended for more than four hours, and sieved. For each of the cans, a weighed amount of plutonium oxide was introduced into the inner can, DE was added, and the lid was then welded shut in a glove box. The inner can was then bagged and placed in the outer can, which was welded shut outside of the glove box. Then each can was blended and vertically scanned to determine the uniformity of the plutonium mixture. If the can did not pass a rigorous uniformity test, blending was repeated. Figure 24 is a photograph of the cans for the standards before the lids were welded. The mass of ${ }^{239} \mathrm{Pu}$ in each can and the total and percentage uncertainty in the mass are listed in Table XVII. 


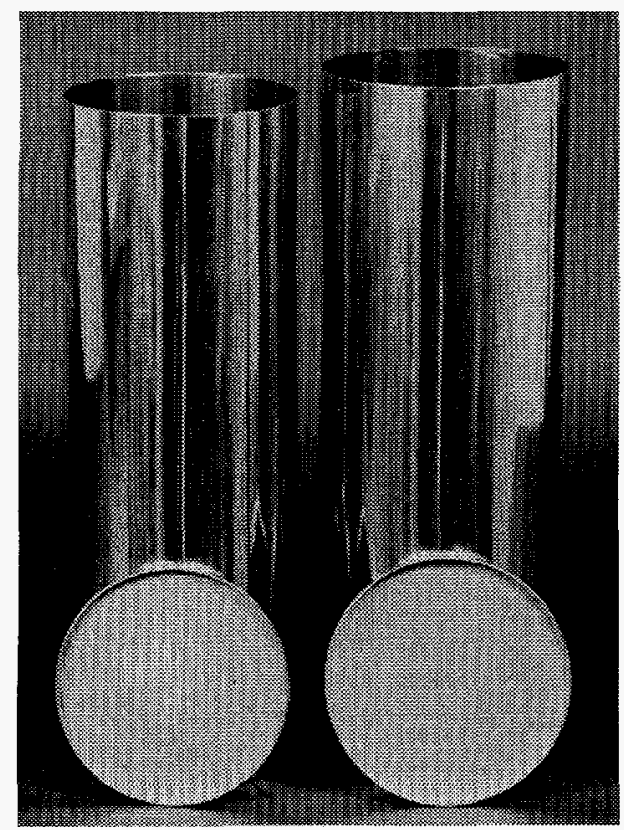

Fig. 24. Cans used for the SGS standards before the welding.

Table XVII

Plutonium Content of SGS Can Standards as of January 1, 1990

\begin{tabular}{|l|l|ll|}
\hline \multicolumn{1}{|c|}{ Standard ID } & ${ }^{239} \mathrm{Pu}$ & \multicolumn{2}{|c|}{ Uncertainty } \\
& $(\mathrm{g})$ & $(\mathrm{g})$ & $(\%)$ \\
\hline & 48.142 & 0.035 & 0.073 \\
STDSGMC-1 & 48.130 & 0.035 & 0.073 \\
STDSGMC-2 & 48.131 & 0.035 & 0.073 \\
STDSGMC-3 & 48.132 & 0.035 & 0.073 \\
STDSGMC-4 & 48.139 & 0.035 & 0.073 \\
STDSGMC-5 & 48.130 & 0.035 & 0.073 \\
STDSGMC-6 & & & \\
& 9.629 & 0.012 & 0.125 \\
STDSGA10 & 28.874 & 0.023 & 0.078 \\
STDSGA30 & 96.269 & 0.068 & 0.071 \\
STDSGA100 & 240.661 & 0.169 & 0.070 \\
STDSGA250 & & & \\
& 9.624 & 0.012 & 0.125 \\
STDSGB10 & 28.890 & 0.023 & 0.078 \\
STDSGB30 & 96.273 & 0.068 & 0.071 \\
STDSGB100 & 192.528 & 0.135 & 0.070 \\
STDSGB200 & & & \\
& 9.625 & 0.012 & 0.125 \\
STDSGC10 & 28.876 & 0.023 & 0.078 \\
STDSGC30 & 96.268 & 0.068 & 0.071 \\
STDSGC100 & 240.664 & 0.169 & 0.070 \\
STDSGC250 & 19.271 & 0.017 & 0.087 \\
& 192.527 & 0.135 & 0.070 \\
STDSGCAL20 & & & \\
STDSGCAL200 & & & \\
\hline
\end{tabular}


Six relatively small samples were selected from different parts of the blended batch and sent to three independent laboratories (NBL, Mound, and Los Alamos) where the samples were characterized. The results were compared to determine the uniformity of the batch and the grams of plutonium per gram of sample. Low-burnup plutonium (96\% $\left.{ }^{239} \mathrm{Pu}\right)$ was used so that the ${ }^{241} \mathrm{Pu}$ component was quite low $(<0.1 \%$ on $1 / 1 / 90)$, which meant that the in-growth of ${ }^{241} \mathrm{Am}$ would be slow. Therefore these standards could be used for a long time before the radiation level would exceed the threshold for precaution in handling.

5. SGS Uranium Drum Standard. To calibrate the Los Alamos SGS drum counter, a set of uranium drum standards was prepared in 1991-1992. It was prepared in the same manner as the SGS can standards except that it was prepared in a modular manner with 4-1 bottles. Normal 200-1 drums are too short to accommodate 4-1 bottles stacked three high. Therefore each drum was lengthened by welding another drum to it. The Cellutex matrix (used because it has approximately the same density as DE) was precut with seven holes and stacked in the drum and then 20 4-1 bottles of uranium oxide were positioned in the drum. Three drums were prepared according to the specifications in Figs. 25 and 26.

The standards were prepared from relatively pure uranium oxide $\left(\mathrm{U}_{3} \mathrm{O}_{8}\right)$ diluted with DE. The feed oxide was high-fired for several hours, sieved through a 100 -mesh sieve to produce a particle size of less than 150 microns, and then blended. Five samples taken from different parts of the blended batch were submitted for chemical characterization. The chemical analysis determined the uranium isotopic composition, the uranium weight fraction, and the loss on ignition. The analysis systems used for chemical determination were calibrated with CRMs.

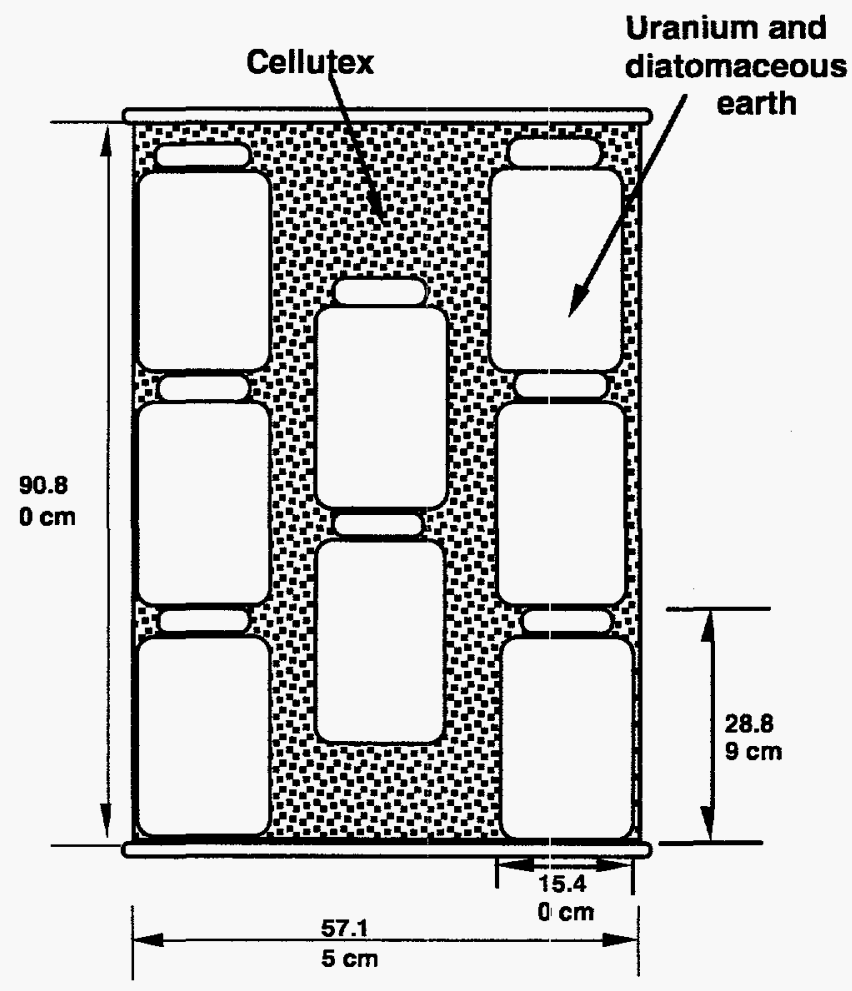

Fig. 25. Side view of the 4-\|l bottles in the drum in a Cellutex matrix. 
Fig. 26. Top view of the 4-1 bottles in the drum.

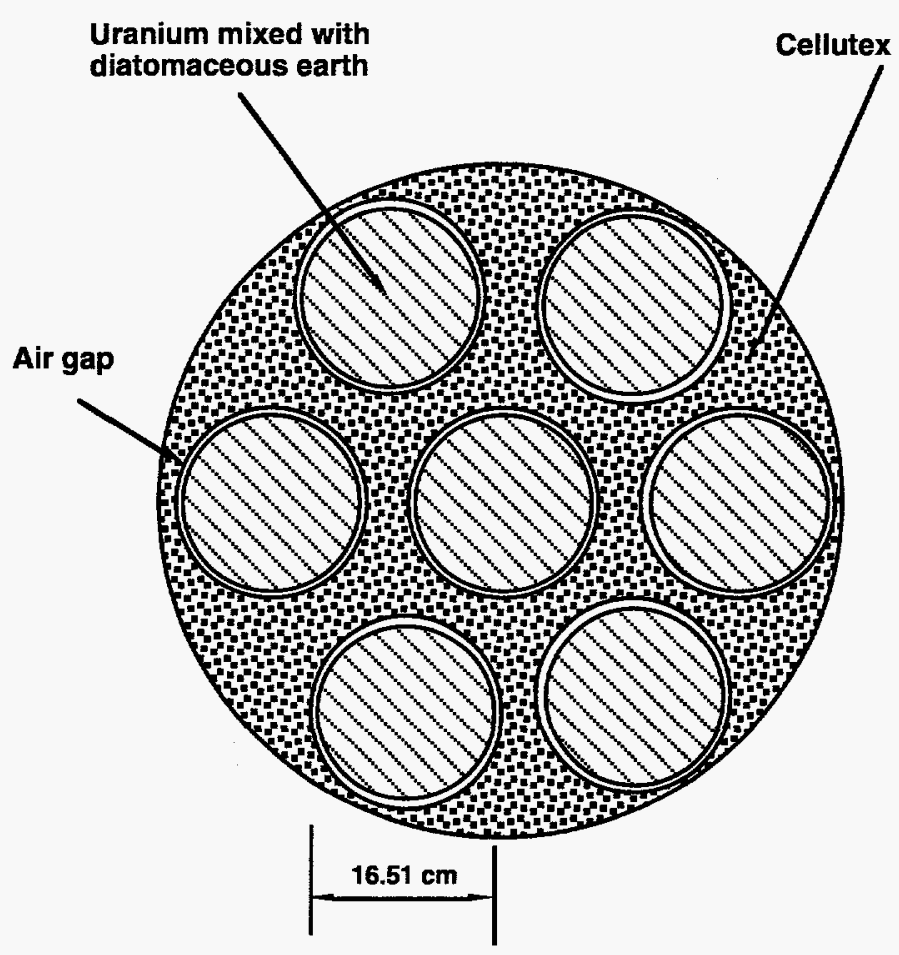

Each bottle was filled with the mixture of uranium oxide and DE and then blended for $60 \mathrm{~min}$. After all the 4-1 bottles were prepared, they were scanned for vertical uniformity with the SGS system. This was performed by measuring the total corrected counts in each segment, from the top of the bottle to the bottom, using the $186-\mathrm{keV}$ gamma peak from ${ }^{235} \mathrm{U}$. The attenuation of each segment was corrected by calculating the correction factor from the transmission measurement. From the vertical scans, eight of the standards were found to be not sufficiently mixed so they were reblended and rescanned.

The certified masses of the three drums are listed in Table XVIII.

One aspect of this set of drum WRMs is less than desirable. With this design, the WRM tends to exacerbate the "end effect," which, if it is not corrected for in the software, will produce a negative bias in the measurement.

6. Active Well Coincidence Counter Uranium Standard. A set of enriched uranium oxide standards was fabricated in 1991-1992 for use with the AWCC.

Table XVIII

Mass and Uncertainty of the Uranium Drum Standards

\begin{tabular}{|c|c|c|}
\hline Standard & ${ }^{235} \mathrm{U}(\mathrm{g})$ & Uncertainty ${ }^{235} \mathrm{U}(\mathrm{g})$ \\
\hline STDSGUD1 & 31.20 & \pm 0.07 \\
\hline STDSGUD2 & 100.99 & \pm 0.21 \\
\hline STDSGUD3 & 200.43 & \pm 0.42 \\
\hline
\end{tabular}


The AWCC has two modes of operation: fast and thermal. In the fast mode, the sample cavity has a cadmium liner that absorbs thermal neutrons; this mode is intended for the assay of large uranium samples. In the thermal mode, the cadmium liner is removed to allow fissions induced by thermal neutrons; this mode is intended for low-mass uranium samples. It was decided that 15 standards could cover the range of sample sizes. These 15 standards can be grouped into low-mass and high-mass sets. The low-mass standards set covers the ${ }^{235} \mathrm{U}$ mass range up to $250 \mathrm{~g}$ and the high-mass set covers the range of $250 \mathrm{~g}$ up to $4 \mathrm{~kg}$. Samples below $250 \mathrm{~g}$ are to be assayed in the thermal mode and those above $250 \mathrm{~g}$ are to be assayed in the fast mode.

Approximately $18 \mathrm{~kg}$ of $\mathrm{U}_{3} \mathrm{O}_{8}$ from four batches were blended into four new bottles by taking approximately the same amount from each batch. These four new bottles were mixed in a blender for one hour. Samples were drawn from each bottle for chemical characterization. The characterization included uranium mass fraction, uranium isotopic distribution, weight loss on ignition (LOI), and impurity determination.

The standards were packaged into food pack cans; two sizes of containers were used to accommodate all the different masses of $\mathrm{U}_{3} \mathrm{O}_{8}$. The smaller cans (A) were $12.75 \mathrm{~cm}$ in diameter by $10.16 \mathrm{~cm}$ tall. The dimensions of the larger cans $(\mathrm{B})$, which used double containment for the higher-mass standards, were $11.43 \mathrm{~cm}$ in diameter by $19.05 \mathrm{~cm}$ tall for the inner can, and $12.75 \mathrm{~cm}$ in diameter by $20.32 \mathrm{~cm}$ tall for the outer can. The masses and the container types for this set of WRMs are shown in Table XIX.

Table XIX

Certified ${ }^{235} \mathrm{U}$ Mass, Container, and Estimated Fill Height of This Set of WRMs

\begin{tabular}{|c|c|c|c|c|}
\hline Standard ID & ${ }^{235} \mathrm{U}(\mathrm{g})$ & Uncertainty $\left({ }^{235} \mathrm{U} \mathrm{g}\right)$ & Container & Est. Fill Height (cm) \\
\hline STDUO10 & 9.98 & \pm 0.02 & A & 0.1 \\
\hline STDUO25 & 25.00 & \pm 0.04 & A & 0.14 \\
\hline STDUO50 & 49.95 & \pm 0.08 & A & 0.27 \\
\hline STDUO75 & 75.00 & \pm 0.12 & A & 0.79 \\
\hline STDUO100 & 99.99 & \pm 0.16 & A & 1.27 \\
\hline STDUO125 & 125.00 & \pm 0.20 & A & 1.43 \\
\hline STDU0150 & 149.99 & \pm 0.23 & $\mathrm{~A}$ & 1.69 \\
\hline STDUO250 & 250.00 & \pm 0.39 & $\mathrm{~A}$ & 2.54 \\
\hline STDUO500 & 500.00 & \pm 0.78 & A & 2.90 \\
\hline STDUO750 & 750.0 & \pm 1.2 & A & 5.08 \\
\hline STDUO1000 & 1000.0 & \pm 1.6 & A & 5.87 \\
\hline STDU01500 & 1500.3 & \pm 2.3 & $\mathrm{~B}$ & 7.62 \\
\hline STDUO2000 & 2000.3 & \pm 3.1 & $\mathrm{~B}$ & 10.2 \\
\hline STDUO2800 & 2800.2 & \pm 4.4 & B & 14.0 \\
\hline STDUO3600 & 3600.5 & \pm 5.6 & B & 18.4 \\
\hline
\end{tabular}


One aspect of this set of AWCC WRMs is less than desirable. For some of the low-mass standards, the fill height is less than $0.5 \mathrm{~cm}$. Because the AWCC is sensitive to the position of the AmLi activation source relative to the sample, any changes in the distribution of the sample in the can could affect the assay result. In retrospect, for the low-mass standards (mass $<50 \mathrm{~g}$ ), a smaller can with the uranium oxide fixed inside the can would be more desirable.

7. Performance Demonstration Program Plutonium Working Reference Material. Of all the WRMs that have been prepared, this set of standards is unique. This WRM set is not intended for the calibration of NDA instruments; rather, it is intended to test the performance of waste assay NDA instruments. It is part of the waste assay performance demonstration program (PDP) organized by the DOE National Transuranic (TRU) Program. The program evaluates the performance of NDA instruments using unknown samples. ${ }^{10}$

The PDP test samples were designed by a team of scientists from different DOE sites so that the samples would be impartial to any particular assay technique. Considerable effort was devoted to using Monte Carlo transport calculations in the design of these samples. These WRMs were designed to provide exact quantities of plutonium and ${ }^{241} \mathrm{Am}$ uniformly distributed in a low-density matrix material. The WRMs were prepared in three sets: one containing $\sim 20-40 \mathrm{mg}$ of plutonium, one containing $\sim 200-400 \mathrm{mg}$ of plutonium and one set containing $\sim 2-4 \mathrm{~g}$ of plutonium. (Exact quantities of plutonium are not revealed to the user to ensure performance testing of the waste assay systems with an unknown.) These samples were inserted into one of three different types of 200-1 drums in a low-density matrix that was a special blend of DE having low limits on impurities that cause $(\alpha, n)$ reactions with plutonium.

The WRMs are contained in stainless steel capsules, doubly contained and independently welded shut. The inner container is cylindrical, $2.45 \mathrm{~cm}$ in diameter and $22.9 \mathrm{~cm}$ high. This allows the insertion of three sources per radial source location, resulting in a total of nine sources to "uniformly fill" the PDP matrix drum. This design is intended for flexibility in drum-loading procedures such that a variety of spatial distributions can be achieved.

Waste generated in nuclear facilities is heterogeneous by nature. It is therefore difficult and expensive to quantify the performance of any waste assay instrument. This set of WRMs has proven to be extremely useful in testing the performance of waste assay NDA instruments.

\section{Conclusions}

We have discussed in this chapter the two CRM standards that are available for the certification of NDA measurements. We have also discussed a variety of WRM standards that exist at PERLA and at Los Alamos. The latter were selected only to illustrate the principles of WRM fabrication and the discussion is by no means complete. 


\section{References}

1. R. J. S. Harry, "Enrichment Standards for Gamma Spectrometry, Historical Overview of the Project to Produce CRM's for ${ }^{235} \mathrm{U}$ Abundance Determination by Gamma-Spectrometry," in Proc. 6th ESARDA Symposium of Safeguards and Nucl. Mater. Manage. (ESARDA, Ispra, Italy, 1984), ESARDA 17, pp. 187-194.

2. P. Matussak, "Accurate Determination of the ${ }^{235} \mathrm{U}$ Isotope Abundance by Gamma Spectrometry: A User's Manual for the Certified Reference Material EC-NRM171/NBS-SRM-969," Insititut für Kernphysik report KfK 3752, Kernforschungszentrum, Karlsruhe, Federal Republic of Germany (1985).

3. S. Guardini, G. Guzzi, F. Mousty, S. Baumann, E. Kuhn, and P. De Regge, "Quality Control Provisions Applied in the Preparation and Characterization of PuBearing PERLA Standards," Institute for Safety Technology, PERLA Safeguards Performance Laboratory, CEC report EUR-13038-EN (1990).

4. S. Guardini, G. Guzzi, F. Mousty, C. Bigliocca, E. Kuhn, N. Doubek, R. Fiedler, A. Zoigner, S. Baumann, K. H. Nelges, G. Hesbacher, P. De Regge, L. Vandevelde, R. Boden, and D. Huys, "Pu PERLA Standards: Quality Control Provisions During Preparation and Analyses of the Samples," in Proc. 7th ESARDA Symposium on Safeguards and Nucl. Mater. Manage. (ESARDA, Ispra, Italy, 1989), ESARDA 19 p. 543.

5. J. Morel and B. Chauvenet, "Intercomparaison des Mesures de Composition Isotopique du Plutonium Par Spectrometrie X Et Gamma Resultats de L'Action 'PIDIE,' Rapport Final," Report CEA-R-5582, Centre D'Etudes Nucleaires de Saclay (December 1991).

6. D. Jackson, R. Wagner, V. Longmire, J. Wachter, F. Hsue, T. Ricketts, S.-T. Hsue, T. Sampson, and J. Stewart, "Report of the NDA Standard Advisory Group," Los Alamos National Laboratory informal report (December 15, 1988).

7. S.-T. Hsue, S. M. Simmonds, V. L. Longmire, and S. M. Long, "Design and Fabrication of SGS Plutonium Standards," in Fourth International Conference on Facility Operations-Sajeguards Interface (American Nuclear Society, La Grange Park, Illinois, 1991), pp. 410-413.

8. F. Hsue, S. M. Long, S.-T. Hsue, and M. C. Miller, "Design and Fabrication of the Uranium Drum Standards," Nucl. Mater Manage. XXII (Proc. Issue), pp. 940-944 (1993).

9. F. Hsue, P. A. Trupp, T. Wilson, E. Vigil, D. Knobloch, and M. Krick, "AWCC Uranium Can Standards Fabrication and Documentation," Los Alamos National Laboratory, Nuclear Materials Measurement and Accountability Group report NMT4-STD1-92 (January 1992). 
10. R. Marshall, M. Foley, L. McCullough, and D. Vance, "Plutonium Working Reference Materials For the NDA PDP Program," presented at the 4th Nondestructive Assay and Nondestructive Examination Waste Characterization Conference," Salt Lake City, Utah, October 24-26, 1995. 


\section{PREPARATION PROCEDURE AND TRACEABILITY}

In Chapters II and III we discussed NDA standards for different types of NDA measurement techniques and that the characteristics of each standard need to be tailored to the NDA technique for which it will be used. We will now present some generalized procedures for the preparation of NDA standards; if the procedures are followed diligently, technically defensible standards that are traceable to the national measurement system will be produced. Most of these procedures follow the recommendations of the American Society for Testing and Materials (ASTM) Standard Guide for Preparation of Working Reference Materials for Use in the Analysis of Nuclear Fuel Cycle Materials, ${ }^{1}$ which covers the preparation of both DA and NDA WRM standards. In this chapter we will point out the specifics applicable only to the preparation of NDA WRM standards. We would like to emphasize that the basis of any NDA WRM is a high-precision chemical analysis that utilizes appropriate CRMs to validate the analytical procedure. A recommended approach to producing WRM standards is given in Fig. 27. In the remainder of this chapter, we will discuss in some detail many of the aspects of producing WRM standards that are listed in Fig. 27.

\section{A. Planning}

The preparation of WRM standards requires careful planning and a written procedure before the work starts to ensure the credibility of the completed WRM. The written procedure should cover the entire project in detail and it should be carefully reviewed to minimize the potential problem areas. It should include at least the following: the intended use of the WRM; the selection and preparation of the SNM; the plans for sampling and sample characterization; containers and packaging for WRM; and the plans for verification measurements and statistical analyses to establish accurately the WRM reference value and its uncertainties.

The starting materials for the preparation of a WRM standard might be SNM material that is already in the desired WRM form. For example, one could take a batch of uranium dioxide pellets or MOX pellets directly from a process run, appropriately sample and characterize it, and then package the batch as a WRM standard. When the starting SNM is not in the desired WRM form, then a different approach must be used to produce the form desired. For example, one could dissolve high-purity uranium metal in acid, add given amounts of impurities to the solution, and then chemically convert the mixture back into the desired uranium dioxide form (after thorough mixing) to produce a WRM standard with a specific level of impurities.

\section{B . Preparation}

The homogeneity of SNM in a batch of nuclear material used to prepare a WRM standard is very important for some obvious reasons. First of all, it is important for the whole batch to be homogeneous in SNM so that all samples selected for chemical analysis 


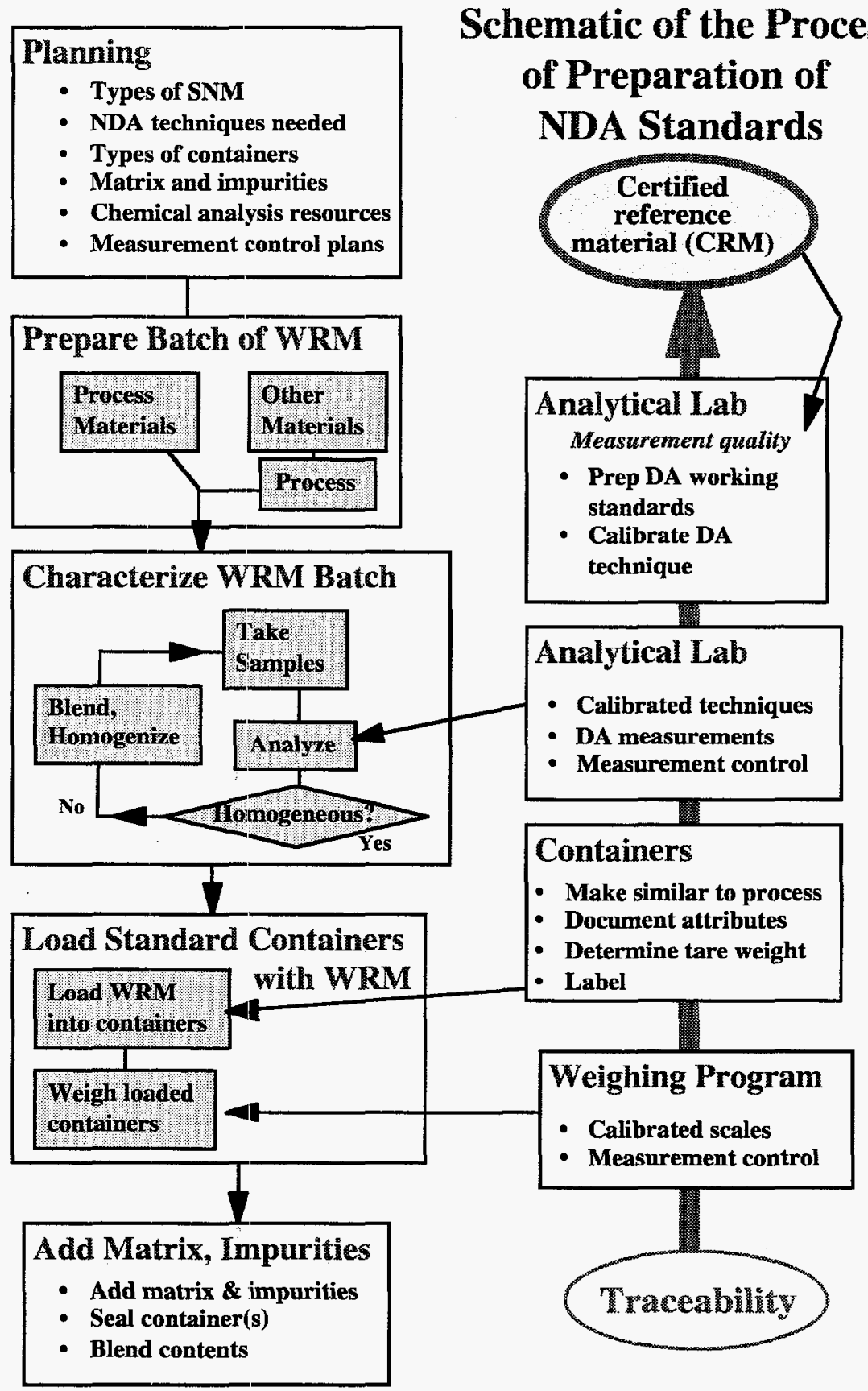

Fig. 27. Recommended procedure to fabricate WRM.

(DA) truly represent the entire batch. Secondly, it is important because it means that any small portion of the standard can be used for a characterization measurement (for example, an isotopic distribution measurement), and the results will represent the whole standard. Normally, homogeneity can readily be accomplished either by chemical methods, such as dissolving the SNM in acid followed by an appropriate precipitation, or by physical methods, such as blending a batch of oxides for an extended period of time (several hours). 
The homogeneity of a batch of SNM can be determined to roughly $1 \%$ by the NDA technique of measuring the isotopic distribution of a small portion of the batch with a collimated detector, or by neutron counting of small samples. A more precise test of homogeneity can be performed by DA techniques, or, if the sample is plutonium, by calorimetry. One can use either of these methods to determine the SNM concentration to a few tenths of a percent. Usually the batch can be considered homogeneous if the results from five or six sample analyses agree within statistical limits.

The form of the nuclear material in a WRM standard can be any stable form of the SNM of interest, but special attention must be given to potential causes of instability, such as problems with chemical reactions, problems with changes in stoichiometry, and problems with radiation damage or radioactive decay. The forms most commonly used for nuclear materials have been oxide powder, pellets, or metals. Once the SNM has been characterized and packaged as a WRM standard (usually in the form of oxide powder, pellets, or in metallic form), it is important that its characteristics remain stable for the estimated shelf life of the standard. This is an important concern because it is well known that regular plutonium oxide is deliquescent and readily absorbs moisture. However, if the oxide has been fired to a relatively high temperature $\left(950^{\circ} \mathrm{C}\right.$ to $\left.1100^{\circ} \mathrm{C}\right)$, then the plutonium oxide is relatively stable and will not absorb moisture. ${ }^{2}$ The moisture content of a sample can be determined by performing the weight LOI test. The LOI test should be performed on every sample taken to determine the plutonium concentration. For the high-fired plutonium oxide, the LOI weight loss usually amounts to only a few tenths of one percent. Once the sample is prepared and sealed (normally under a dry nitrogen atmosphere), any subsequent weight gain has been found to be negligible. This obviously means that the standard must be very well sealed to prevent moisture or other impurities from seeping into the container.

\section{Weighing}

The final determination of the amount of SNM in a WRM standard is carried out by weight measurements because they constitute such a high-precision measurement method. Therefore, it is important that the weight be determined accurately and correctly. The scale or balance should be properly chosen to accommodate the range of weights to be determined and it should be at least a factor of 10 more accurate than the final requirements. The balance should be verified with NIST-traceable certified weights both before and after the weighing of the SNM. The certified weights and the SNM samples should be weighed by one person with a second person to verify the weight readings to minimize transcription errors in recording the weights.

An important aspect of weighing in the preparation of WRM standards is to determine the appropriate time in the procedure to make the weight measurements. Let us take the case of preparing a diluted plutonium oxide WRM standard for the NDA technique of SGS. There are two potential methods of determining the weight of SNM. In the first method, the empty inner can is initially weighed, an appropriate quantity of plutonium oxide is transferred to the can, and then the can is weighed again to determine the net weight of plutonium oxide. Then the diluent material (typically graphite DE) is added to the 
can, and after the can is properly sealed, the mixture is blended by shaking to create a homogeneous mixture. This method requires that the plutonium concentration of the oxide be determined by DA techniques. In the second method, an already diluted and homogeneous plutonium oxide mixture is transferred into the preweighed inner can. This method requires that the plutonium concentration of the diluted oxide be determined by DA techniques, which is potentially a much harder problem, especially if the diluent material cannot be readily dissolved. Graphite and DE are commonly used as diluents in the preparation of such standards; both are difficult to dissolve by standard chemical techniques.

\section{Container and Packaging}

It is important that the container material be chemically compatible with the WRM matrix and that the container material not affect signals from the WRM. For example, plastic bottles should not be used for neutron standards because plastics tend to moderate the neutron spectrum and thereby affect neutron measurements. Some plastics also contain fluorine, which will increase the $(\alpha, n)$ signal from the standard.

Usually, aluminum, steel, or stainless steel containers should be used to package the WRM standards. Steel (food) cans that can be sealed by a food packing process are sometimes used, but food pack cans are nominally, relatively thin and tend to deform with usage. Aluminum and stainless steel are better choices for container materials, but both require welding to seal the container, which is cumbersome and costly if it has to be done inside a glove box (for plutonium WRM). Few facilities can weld inside glove boxes. Whenever a stainless steel container is used for a gamma-ray WRM, the attenuation of the gamma rays due to the container should be taken into account in the calibration of the NDA instrument being used.

After the container is packaged, it should also be leak tested to minimize any possibility of radioactive contamination leaking out or moisture and other impurities leaking into the standard.

\section{E. Sampling and Characterization}

Of all the steps in the preparation of WRM standards, the sampling and subsequent sample characterization is the most important step and it needs to be planned carefully. It is the combination of sampling and characterization that enables one to determine both the uniformity of the batch of WRM and the amount of nuclear material in each standard. Samples characterized by a trained analyst are traceable to the national system of measurements.

Sampling is relatively simple if the WRM is uranium, plutonium, or mixed oxide. Typically, samples are taken from different parts of a mixed and blended oxide batch to test homogeneity. Sampling can become complicated if the WRM is in the form of fuel pellets. But one can select fuel pellets from the same batch, then assure the uniformity from pin to pin by measuring the U/Pu ratio or the oxygen to heavy metal ratio, and finally select several fuel pins for DA.

Figure 28 is a block diagram of the United States nuclear measurement system. Because the available types of CRMs for the calibration of NDA techniques are limited 


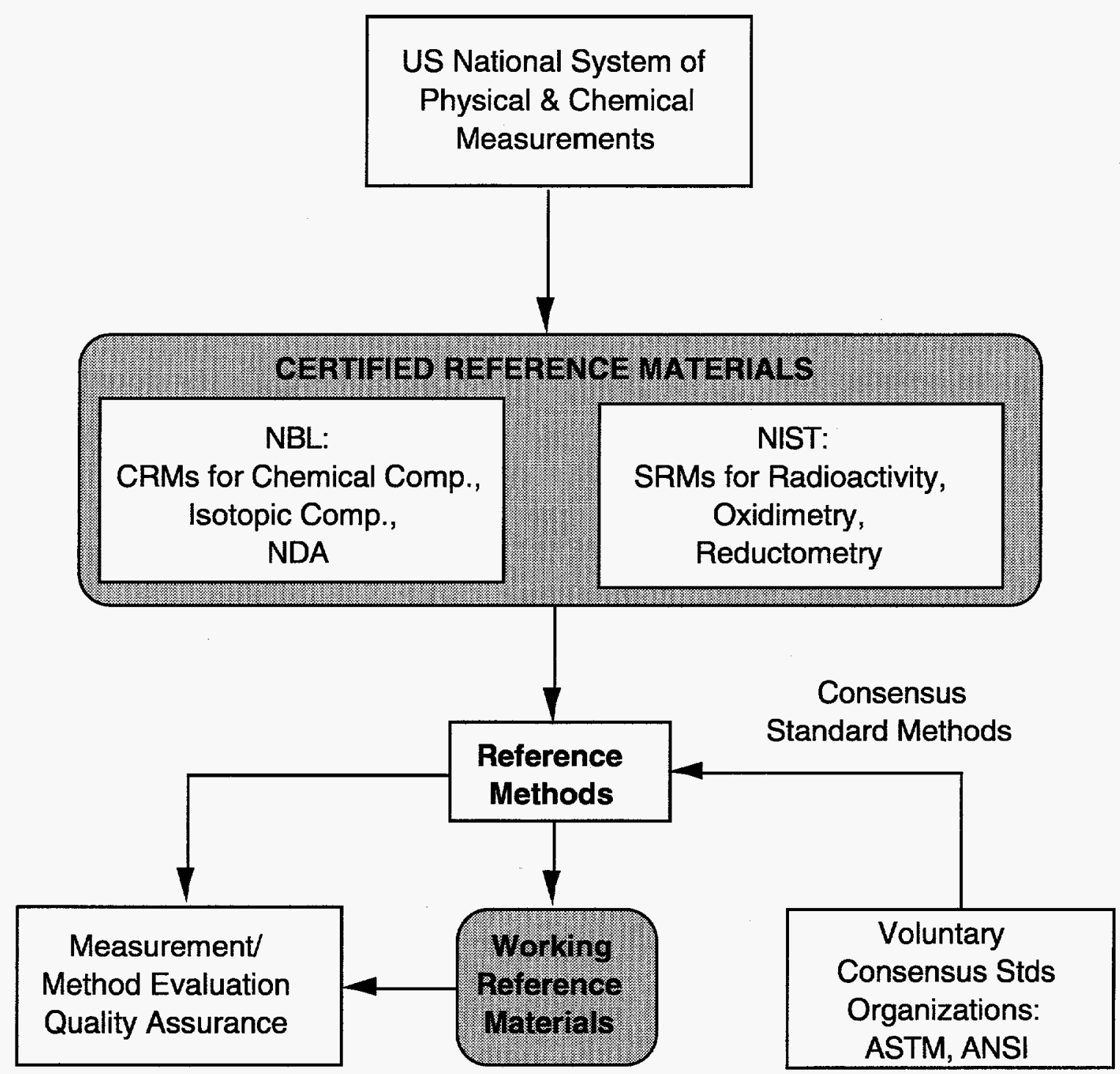

Fig. 28. United States nuclear measurement system.

(Chapter IV), the traceability of the WRM has to rely mainly on DA techniques. Where possible, DA CRM standards are used to calibrate the methods used to establish the concentration values (reference values) assigned to the WRMs, thus providing the traceability to CRM standards. Uranium- and plutonium-concentration CRM standards are available from NBL as well as CBNM in Europe. Uranium and plutonium isotopic CRM standards are also available from NBL and CBNM. ${ }^{3}$ The analytical laboratories should validate their measurement techniques by assaying the CRM standards before or after assaying the unknown nuclear material. The analysis method selected should be a consensus standard method for the sample to be analyzed. For example, mass spectrometry should be used to determine the ${ }^{239} \mathrm{Pu}^{, 240} \mathrm{Pu}^{241} \mathrm{Pu}^{242} \mathrm{Pu}$ isotopic distribution, 
and alpha spectrometry should be used to determine the ${ }^{238} \mathrm{Pu}$ and ${ }^{241} \mathrm{Am}$ isotopic distribution. The uranium concentration of a sample can be determined by means of the Davis-Gray technique. Because of the importance of DA and its traceability to the national measurement system, it is desirable to have two or more independent laboratories performing analyses on identical samples from the same batch of WRM. This is necessary to prevent any potential systematic bias from one laboratory. However, the desire to minimize systematic bias must be balanced with the additional cost and the time delay.

There is also the question of how accurate the DA should be. A general guideline is that the results of the DA should be more precise by a factor of 3 to 5 than and less biased compared to the normal accuracy of the NDA technique with which the WRM will be used. For example, in preparing a neutron standard for an NDA technique where the measurement precision is typically $1 \%$, the DA to quantify the WRM should have a precision of $\sim 0.2 \%$ so that the uncertainty contributed by the DA to the overall NDA measurement error is relatively small, $0.02 \%$ in this case. In the case of waste WRM standards, the DA can be more precise by a factor of 3. For example, an analytical DA precision of $1 \%$ to $2 \%$ is acceptable for SGS WRM standards because the SGS assay precision and bias are typically around 5\%. In light of this, it is quite possible that a combination of calorimetry and isotopic distribution measurements will be sufficient to quantify WRMs intended for NDA measurements on waste nuclear material. The advantage of this approach is that it is relatively inexpensive compared to DA techniques.

At least one of the samples should be analyzed for impurities to limit the influence of the impurities on the certification of the WRM. Typical low-Z impurities that can have a marked effect on neutron NDA are beryllium, boron, fluorine, lithium, sodium, magnesium, aluminum, silicon, chlorine, carbon, and oxygen. Some samples should also be archived to facilitate the resolution of any future questions concerning the standard ("referee" samples).

\section{F . Verification}

For a set of WRM standards that covers a range of masses (or concentrations), it is necessary to perform verification measurements to check the internal consistency of the set. This is particularly important for solution WRM standards. Solutions are ideal samples and NDA systems for solutions can achieve an analytical precision and bias of $0.1 \%$ to $0.2 \%$. We have found that NDA techniques can determine the gross inconsistencies within a set of solution WRM standards that range from low to high concentrations. The total corrected counts/g of SNM should be internally consistent for all of the WRM standards in a set of standards; we have found that some samples from a set of concentration WRM standards can deviate from the average value for the amount of SNM by several percent. An example of the verification measurements for a set of SGS can standards ${ }^{4}$ is shown in Fig. 29. 


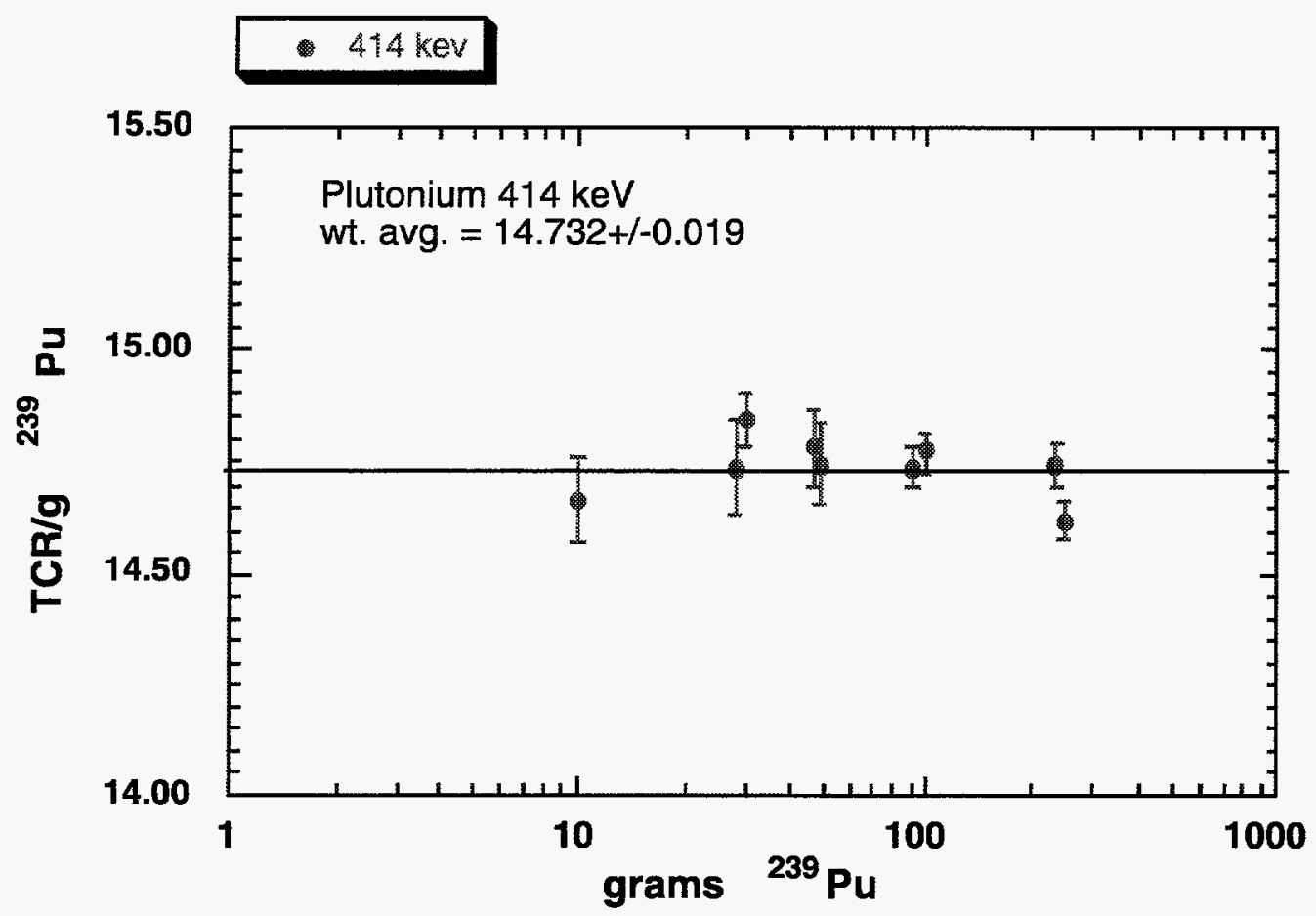

Fig. 29. Measurements for set $A$ of the SGS can standard plus one of the measurement control standards.

\section{Conclusion}

We have outlined in this chapter the recommended steps to prepare traceable WRMs in procedures that have been approved by the ASTM. It should be emphasized that the foundation of the preparation of an NDA WRM is an accurate chemical analysis that has been validated with CRM standards. If the procedures are followed diligently, technically defensible standards that are traceable to the national measurement system will be produced. While these steps are necessary, they may not be sufficient. To produce CRMs, it is very important to have a thorough understanding of the scientific principles involved in both DA and NDA techniques.

\section{References}

1. "Standard Guide for Preparation of Working Reference Materials for Use in the Analysis of Nuclear Fuel Cycle Materials," American Society For Testing and Materials, Designation C1128-89, ASTM, (1989).

2. S. Guardini, G. Guzzi, F. Mousty, S. Baumann, E. Kuhn, and P. De Regge, "Quality Control Provisions Applied in the Preparation and Characterization of $\mathrm{Pu}$ Bearing PERLA Standards," Institute for Safety Technology, PERLA Safeguards Performance Laboratory, CEC report EUR-13038-EN (1990). 
3. New Brunswick Laboratory, US Department of Energy, 9800 South Cass Avenue, Argonne, IL 60439-4899.

4. S.-T. Hsue, S. M. Simmonds, V. L. Longmire, and S. M. Long, "Design and Fabrication of SGS Plutonium Standards," in Fourth International Conference on Facility Operations-Safeguards Interface (American Nuclear Society, La Grange Park, Illinois, 1991), pp. 410-413. 


\section{ALTERNATIVES TO STANDARDS}

\section{A. Detector Family Techniques-Cross Calibration}

For both domestic and international inspections of nuclear materials, it is often necessary to employ NDA instruments based on neutron time-correlation counting. For quantitative NDA, the neutron-measuring instruments must be calibrated using physical standards representative of the unknowns. For four reasons, calibration requirements and constraints are significantly different for international-inspector-controlled NDA equipment than for in-plant operator equipment: 1) the physical standards representative of the assay samples are generally not available to the inspector at the plant; 2) the inspector normally covers a much wider range of nuclear materials than does the plant operator because the inspector visits many different facilities; 3) the inspector typically uses a family of similar NDA instruments rather than a single, specialized detector; and 4) the inspector routinely transports equipment between facilities, making it necessary to frequently renormalize prior calibrations.

The traditional calibration approach is to develop physical standards representative of the unknowns and then measure them on the same detector that will be used for the unknowns. However, this approach is frequently impractical for field verification, e. g., because the standards must be sampled and the samples destructively analyzed for certification. Calibration and normalization procedures have been developed for the Uranium Neutron Coincidence Collar (IAEA designation $\mathrm{UNCL}^{1}$ to overcome the above-mentioned problems. These UNCL calibration procedures can be generalized for the other instrument families such as the AWCC. ${ }^{2}$ Both the AWCC and the UNCL are active systems and hence they are more complex to implement than the passive systems such as the High-Level Neutron-Coincidence Counter (HLNC-II). ${ }^{3}$

The basic idea of cross-calibration ${ }^{4}$ is to carefully calibrate one member of the instrument family for an important category of material [for example, boiling-water reactor (BWR) fuel assemblies] covering a wide range of mass loadings. The calibration parameters for this reference detector are then fixed, and the responses of other members of the instrument family are normalized to these fixed calibration parameters.

This technique of fixing calibration parameters (curve shape) assumes that the nonlinear shape is primarily a characteristic of the nuclear material items, and any detector-related effects are the same for all members of that instrument family.

The primary benefit of this calibration approach is that it reduces the requirements for physical standards as well as the in-field time required to completely calibrate all members of a detector family. For example, there are presently more than $20 \mathrm{UNCLs,} \mathrm{and} \mathrm{it} \mathrm{is} \mathrm{very} \mathrm{difficult} \mathrm{to}$ find a nuclear facility with a wide enough range of BWR or pressurized-water reactor (PWR) mass loadings. Also, full use is made of historical calibration data. This tie-in with historical data enhances the quality assurance of measurements. Another more subtle benefit is that detector-todetector consistency can be determined easily using the cross-reference approach.

The following sections give specific examples and recommended calibration functions for selected detector families.

1. Inventory Sample Counter ${ }^{5}$. The case of the inventory sample (INVS) counter is considered first, because of its simplicity. There are only two material categories. Multiplication corrections for both are insignificant and therefore unnecessary. 
The material categories of interest are

1. $\mathrm{PuO}_{2}$ powder, $\mathrm{MOX}$ powder, and pellets; and

2. plutonium nitrate and other solutions.

In all cases, the sample plutonium masses should be $<20 \mathrm{~g}$ for solids and $<10 \mathrm{~g}$ for liquids. It is possible to lump several material types into one category because the neutronmultiplication differences are negligible.

The preferred calibration function is

$$
R=a_{1} m+a_{2} m^{2}
$$

where $R$ is the real coincidence count rate (Reals), $a_{1} m>>a_{2} m^{2}$, and $m$ is the effective ${ }^{240} \mathrm{Pu}$ mass.

Note that the term $a_{2} m^{2}$ represents the small multiplication effects.

A set of physical standards covering the full mass range of interest is used to obtain the constants $a_{1}$ and $a_{2}$ for the reference $\mathbb{I N V S}$. The reals rate for a reference ${ }^{252} \mathrm{Cf}$ spontaneous-fission neutron source is measured at the same time as the calibration of the reference INVS. The precision of the ${ }^{252} \mathrm{Cf}$ reals measurement should be $0.5 \%$, or less. The ${ }^{252} \mathrm{Cf}$ data, based on measurements made with the Los Alamos source CR-5, are given in Ref. 1 (Table I, p. 4) for seven INVS counters. The calibration parameters $a_{1}$ and $a_{2}$ are also given for MOX pellets and powders measured in the reference INVS.

For in-field applications of the reference INVS, the measured response $R$ is corrected for possible electronic drifts by remeasuring the reference ${ }^{252} \mathrm{Cf}$ source (or one whose relative neutron intensity is known), making decay corrections, and computing the electronics normalization constant. Table XIX of Ref. 1 gives absolute and relative yields for $43{ }^{252} \mathrm{Cf}$ sources used routinely by Los Alamos, the IAEA, and EURATOM.

A different INVS can be cross-calibrated by counting the same reference sample (or samples) in both the reference and new detectors at approximately the same time and computing the cross-calibration constant. Occasionally, a container correction is required.

The complete correction factor is

$k=($ electronics $) \cdot($ cross reference $) \cdot($ container changes $)$, or

$k=\left[R_{0}(C f) / R_{\text {new }}(C f) \cdot\left[R_{0}(\mathrm{INVS}-\mathrm{ref}) / R_{0}(\mathrm{INVS}-\mathrm{X})\right] \bullet(\right.$ container changes $)$

The cross-reference term $R_{0}$ (INVS-ref)/ $R_{0}$ (INVS-X) can be measured using either a plutonium sample or a ${ }^{252} \mathrm{Cf}$ source. The counting precision should be a few tenths of a percent, or less, because any error in this factor will appear as a bias in the assay.

The last term in $k$, which represents container or matrix changes or both, is needed only if the unknown samples are packaged in a way that alters their INVS response relative to the original standards. Usually, this term is unity. 
Given the stability of present-day coincidence electronics and power supplies, the electronics term will be near unity under most conditions.

After $k$ has been determined, the new calibration equation is

$$
k R=a_{1} m+a_{2} m^{2}
$$

The significant aspects of using the cross-calibration procedure are to introduce the term $R_{0}$ (INVSref) $/ R_{0}$ (INVS-X) and to fix the calibration constants $a_{1}$ and $a_{2}$ of all INVS detector heads for a given material category.

2. High-Level Neutron-Coincidence Counter. ${ }^{3}$ The HLNC-II is a more complicated case than the INVS because of the significance of neutron multiplication effects in larger samples. There are two possible calibrations: $R$, before multiplication corrections and $R_{m c}$, after multiplication corrections. ${ }^{6} \quad$ The calibration function for $R$ vs $m$ (effective ${ }^{240} \mathrm{Pu}$ mass) changes for different sample sizes, densities, isotopics, and impurities. Thus the calibration function

$$
R=a_{0}+a_{1} m+a_{2} m^{2}
$$

is very sample-dependent, and considerable scatter is observed among different samples because of the effects mentioned above.

On the other hand, if $\alpha[(\alpha, n)$ neutrons/spontaneous-fission neutrons produced in item] is known, then the calibration function

$$
R_{m c}=a m
$$

is valid over a wide range of sample characteristics. The constant $a$ defines the base-line slope of the multiplication-corrected calibration. This is a constant for a particular HLNC-II (typical values are $a=18.15$ for $\rho_{0}=0.103$ ), and the ratio of $a / \rho_{0}=176$ is proportional to the detector efficiency. The constant $\rho_{0}$ is defined as $R / T(1+\alpha)$ for a nonmultiplying sample. For a typical HLNC-II, the effective ${ }^{240} \mathrm{Pu}$ mass can be written

$$
m=T / 176(1+\alpha) M]
$$

where $T$ is the total neutron count rate and $M$ is the sample leakage multiplication. The variable $M$ is calculated from the $R / T$ ratio, with a known value for $\alpha$. The parameter $\alpha$ is 0 for pure plutonium metal and calculable for pure plutonium oxide (or impure oxide, only if the impurity concentrations are known) with known plutonium isotopic ratios. For cross-calibration, it is necessary to correct both $R$ and $T$ before calculating $M$ or using the calibration constant $a$.

The correction $k$ for the HLNC-II is defined the same as $k$ for the INVS: 
$k=\left[R_{0}(C f) / R_{\text {new }}(C f)\right] \cdot\left[R_{0}(\mathrm{HLNC}-\mathrm{ref}) / R_{0}(\mathrm{HLNC}-\mathrm{X})\right] \bullet$ (sample correction).

The calibration equations become

$$
\begin{aligned}
& k R=a_{0}+a_{1} m+a_{2} m^{2}, \text { and } \\
& R_{m c}=a m
\end{aligned}
$$

The totals rate $T$ is corrected by $k^{0.5}$ because changes in the efficiency $\varepsilon$ affect $R$ as $\varepsilon^{2}$ and $T$ as $\varepsilon$. $R$ and $T$ are corrected by $k$ and $k^{0.5}$, respectively, before the $R / T$ ratio is used to calculate $R_{m c}$ and $M$. These renormalization corrections are performed automatically in present-day neutron coincidence counting software.

Reference values for 19 members of the HLNC-II family are given in Table III of Ref. 4. Ref. 4 also gives uncorrected and multiplication-corrected HLNC-II calibration parameters determined from 39 bulk $\mathrm{PuO}_{2}$ powder samples covering the mass range of 60 to $7000 \mathrm{~g}$ of plutonium. Use of the uncorrected $R$ calibration curve is limited to this mass range. Samples with different sizes, densities, and isotopics will scatter about the $R$ curve, and the $k$ factor does not address this scatter. The $R_{m c}$ calibration data do not scatter because effects due to size, density, and isotopic variations are corrected. After the $k$ correction is made, all HLNC-II systems will have the same linear calibration line, $R_{m c}=18.15 \mathrm{~m}$. If one compares the calculated $M$ with that predicted from Monte Carlo simulations, we have $\rho_{o}=0.108$, and $a=19.05$.

The primary benefit of this calibration procedure is that all HLNC-II units have the same calibration equation for the same material category, and new units can be cross-referenced using a single sample or even a ${ }^{252} \mathrm{Cf}$ source. To preserve the accuracy of this simple normalization method, deadtime corrections must be accurate so that the shape of the calibration curve is not distorted at the higher counting rates. Also, totals background corrections must be accurate because of the use of the totals rate in the multiplication. This can require frequent background measurements in situations where the background is significant and variable.

3. Active-Well Coincidence Counter. ${ }^{2}$ The AWCC is an active assay system for which the measured response $R$ is more complex than for the two previous passive instruments. In addition to the passive counting efficiency, the response is dependent on the neutron interrogation flux from the AmLi random sources. The observed coincidence response $R$ is the product of the sample characteristics, the flux, and the square of the counting efficiency. This means that when we calibrate and cross-reference different detectors, we must normalize for AmLi interrogation sources with different neutron intensities.

To use the same normalized calibration function for a detector family, we have heretofore assumed that the detector heads have identical materials, geometry, and flux profiles. Thus, the AWCC normalizations must be made for each end-plug configuration, because changing the end plugs changes the neutron flux profile and the AmLi source reference rate $\left(T_{0}\right)$. It is clear that we need to keep the end-plug variations to a minimum to avoid undue complexity.

For most field applications, a ${ }^{252} \mathrm{Cf}$ source can be used to correct for any changes in detector efficiency using the same procedure as for the HLNC-II. However, for some field applications of the AWCC and the UNCL, ${ }^{252} \mathrm{Cf}$ sources are not available, so the AmLi(s) are used to normalize the detector counting efficiency. Because the calibration response is the real coincidence rate $R$, the reference net AmLi total count rate is squared to obtain the appropriate 
correction factor. Whenever a ${ }^{252} \mathrm{Cf}$ reference source is available, it should be used for crossreferencing instead of the AmLi source(s). Out of necessity, it must be assumed that the interrogation source coupling to the sample does not change with time. The interrogation flux can be normalized using a uranium reference sample. This procedure checks both the flux and the counting efficiency.

Various calibration functions have been obtained for the AWCC, and it is evident that a function with an inflection is necessary to cover a large mass range. This arises from selfshielding (negative curvature) in the low-mass range and neutron multiplication (positive curvature) in the high-mass range.

For illustration purposes, a cubic polynomial with the possibility of a nonzero intercept (at zero mass) will be assumed. For some calibration data sets, the inflection is not present and the coefficient of the cubic term can be set to zero. In general,

$$
k R=a_{0}+a_{1} m+a_{2} m^{2}+a_{3} m^{3} .
$$

For a different AWCC or the same AWCC (reference unit) at a different time, the normalization factor is

$$
k=\left[R_{0}(C f) / R_{\text {new }}(C f)\right] \bullet\left[R_{0}(\mathrm{AWCC}-\mathrm{ref}) / R_{0}(\mathrm{AWCC}-\mathrm{X})\right] \bullet(\text { sample correction }),
$$

or when using an AmLi source if no ${ }^{252} \mathrm{Cf}$ reference source is available, it is

$$
k=\left[T_{0}(\mathrm{AmLi}) / T_{\text {new }}(\mathrm{AmLi})\right]^{2} \cdot\left[R_{0}(\mathrm{AWCC}-\mathrm{ref}) / R_{0}(\mathrm{AWCC}-\mathrm{X})\right] \bullet(\text { sample correction })
$$

The ratio $\left.R_{0}(\mathrm{AWCC}-\mathrm{ref}) / \boldsymbol{R}_{0}(\mathrm{AWCC}-\mathrm{X})\right]$ corresponds to the responses from the two units measuring the same uranium sample with their assigned AmLi sources.

Both the $T_{0}$ and $R_{0}$ values must be corrected for ${ }^{241} \mathrm{Am}\left(T_{1 / 2}=432 \mathrm{yr}\right)$ and ${ }^{252} \mathrm{Cf}\left(T_{1 / 2}=\right.$ $2.643 \mathrm{yr}$ ) decay. A correction must also be applied to the measured value of $R$ because of $\mathrm{AmLi}$ source decay. For consistency, all source-decay dependent quantities should be corrected back in time to the date of original calibration.

The sample correction term allows correction for known biases caused by differences between the calibration samples and the unknowns. An example is calibration with $\mathrm{UO}_{2}$ in steel cans and assay of $\mathrm{UO}_{2}$ in polyethylene bottles, which will increase $R$ by a few percent. This perturbation can be measured or calculated and applied to $k$.

Material categories that require individual calibrations include

1. HEU metal disks or buttons,

2. HEU metal chips or pieces,

3. U-Al bildchen,

4. U/Th/C beads,

5. $\mathrm{U}_{3} \mathrm{O}_{8}$ powder,

6. $\mathrm{UF}_{4}$, 
7. Materials Testing Reactor (MTR) elements,

8. $\mathrm{U} / \mathrm{Th} / \mathrm{C}$ pebbles, and

9. $\mathrm{UO}_{2}$.

The most significant factor resulting in different material categories (and thus calibration curves) is the ${ }^{235} \mathrm{U}$ density $\left(\mathrm{g} / \mathrm{cm}^{3}\right)$.

4. Uranium Neutron Coincidence Collar. ${ }^{1}$ UNCLs are being routinely used for inspection activities by both the IAEA and the Commission of European Communities (CEC) Safeguards Directorate, Luxembourg (EURATOM). These activities have led to increased requirements for calibration of all the collars in use and a procedure to apply the calibrations to differing fuel element types. The UNCL case is the most complex case to apply the crosscalibration approach, but the UNCL derives the most benefit because of the relative unavailability of fresh light-water reactor (LWR) fuel elements that can be used as standards. Compared with the AWCC, only one AmLi source is used, thus the problem of unmatched sources is not present. However, additional corrections are required because LWR fuel elements include many different enrichments, pin configurations, fuel masses, and burnable poison loadings. The case of the UNCL is treated comprehensively in Ref. 1 and thus won't be elaborated here.

5. Cross-Calibration Summary. The primary goal of the calibration crossreference approach described in this section is to reduce the number of physical standards and the time required for calibration work. The larger the number of detectors in a family, the greater the savings. Standards from several different facilities can be used to better define calibration curve shapes over a wide range of fissile loadings. The cross-reference approach also makes it much easier to spot instrument malfunctions and sample outliers.

Although the method was first introduced for the UNCL active assay system, the basic method can be used with greater ease and better accuracy with the passive systems such as the HLNC-II and INVS.

The AWCCs do not meet the full assumptions for applying the cross-reference approach because the various detector heads can have different response functions caused by unmatched AmLi sources. For example, if the top source is more intense than the bottom source, the interrogation flux profile will be distorted, changing the shape of the calibration curve.

The cross-reference ratios and the calibration coefficients given in Ref. 4 represent a first approximation to the final values. As more accurate calibration data are obtained, updates are being made.

One of the basic problems in selecting the appropriate calibration functions such as polynomials, power functions, or exponentials is selecting a reliable and representative data set. The cross-reference approach helps to solve this problem if the reference detector and the primary calibration data cover the full mass range of interest. Before the primary calibration function can be obtained, the data should be

1. free of anomalous data (e.g., electronic noise or badly characterized standards),

2. accurately corrected for dead time,

3. accurately corrected for container variations, and

4. free of sample positioning and fill-height effects. 
The normalization constant $k$ must be applied carefully for standard sets that span different facilities and times. When there are known sample-to-sample differences within the sample category, corrections for these differences must be made to $k$ before curve fitting. The magnitude of the sample perturbation can be determined using Monte Carlo simulations or experimental measurements or both.

Calculational results using Monte Carlo computer codes can be applied directly to the sample correction factor in $k$ to improve assay accuracy. This topic is the subject of the next section. The sample-dependent correction term in $k$ can be used to correct any known biases in the calibration equations.

\section{B. Use of Monte Carlo Simulations to Minimize the Required Number of Standards}

In several important cases, sufficient information is known about the items to be assayed so that accurate simulations of instrument response can be constructed. The first of these cases is that of unirradiated fast breeder reactor (FBR) fuel elements. One of the primary assay techniques for this important material category is passive neutron coincidence counting (PNCC). Another is active neutron coincidence counting (ANCC) of highly enriched uranium (HEU) in the form of $\mathrm{UF}_{6}$. A third case is of ANCC applied to fissile assay of research reactor irradiated fuel elements. These cases are described in the following three subsections. Other cases also exist and some are being actively pursued.

1. Fast Breeder Reactor Mixed-Oxide Fuel Elements. In Ref. 7, a calculational model is applied to the two-parameter (singles and doubles) PNCC assay of fresh, finished FBR subassemblies and the results are compared with calibration measurements. Two assay instruments were considered: the Universal Fast Breeder Reactor Subassembly Counter (UFBC) and the Capsule Counter installed in the Japanese Plutonium Fuel Production Facility. Passive UFBC assays of four Fast Flux Test Facility (FFTF) and six German SNR-300 FBR fuel subassemblies were simulated. Calculated results are shown in Fig. 30, along with measured

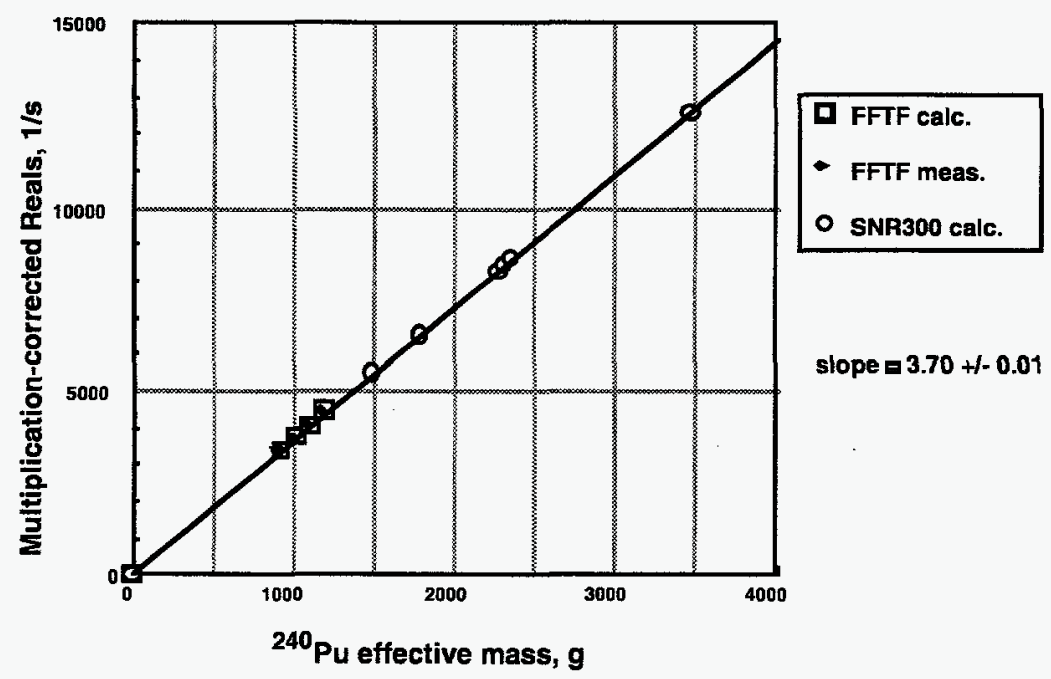

Fig. 30. Plot of calculated and measured multiplication-corrected real-coincidence count rates versus effective ${ }^{240} \mathrm{Pu}$ mass for FFTF and SNR300 FBR fuel subassemblies measured in the UFBC. The linear fit (through the origin) is that for the calculated points and its slope is $3.70 \pm 0.01$. 
results for the FFTF assemblies. Passive Capsule Counter assays of five FFTF, three JOYO assemblies, and one MONJU assembly were simulated. Calculated and measured results are shown in Fig. 31.

For the case of the US FFTF fuel, the absolute ratio of calculations to measurements for the multiplication-corrected-reals calibration constant is $+1.1 \pm 1.0 \%$ (average of four subassemblies) for the UFBC and $-1.3 \pm 0.6 \%$ (average of five subassemblies) for the Plutonium Fuel Production Facility (PFPF) Capsule Counter. For initial measurements of Japanese fuel in the Capsule Counter, the absolute ratio is $-1.0 \pm 0.7 \%$ for three JOYO assemblies and $+0.8 \pm 0.7 \%$ for the one MONJU assembly. The calculated results are very accurate and precise and offer more effective and less costly inspector verification of FBR fuel elements by reducing reliance on physical standards (as well as costly and time-consuming sampling and DA) to expand the cross-calibration database.

Fig. 31. Plot of calculated and measured multiplicationcorrected real-coincidence count rates versus effective ${ }^{240}$ Pu mass for FFTF, JOYO, and MONJU FBR fuel subassemblies measured in the PFPF Capsule Counter. The linear fit (through the origin) is that for the calculated points and its slope is $14.79 \pm 0.02$.

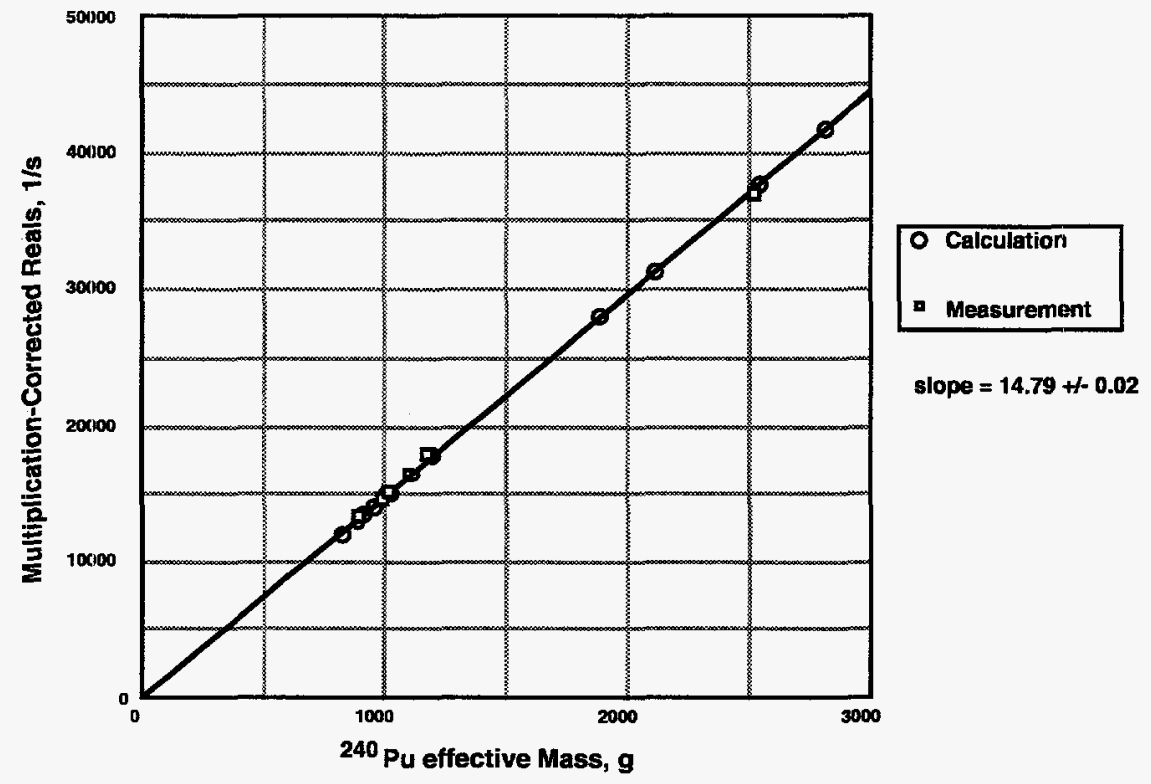

2. Uranium Hexafluoride $\left(\mathbf{U F}_{6}\right)$. An AWCC was modified to measure variableenrichment $\mathrm{UF}_{6}$ in storage bottles. ${ }^{9}$ An active technique was used to assay the ${ }^{235} \mathrm{U}$ content because of the small quantity (nominal loading of $2 \mathrm{~kg} \mathrm{UF}_{6}$ ) and nonuniform distribution of $\mathrm{UF}_{6}$ in the storage bottles. A new AWCC insert, composed of graphite containing four AmLi sources, was designed. Monte Carlo calculations were used to design the insert and to calibrate the detector. Benchmark measurements and calculations were performed using uranium oxide standards. The Monte Carlo generated calibration curves, normalized to uranium oxide measurements, resulted in $\mathrm{UF}_{6}$ assays that agreed to within $2 \%$ to $3 \%$ of the $\mathrm{DA}$ values. In addition, the AWCC was also calibrated for HEU ingots, billets, and scrap alloy using the standard end-plug configuration for fast model $1 .^{4}$

3. Irradiated Materials Test Reactor Fuel Elements. A special, ANCC was designed, built, and tested at the SRS for the assay of irradiated MTR fuel elements. The instrument operates underwater and is called the Research Reactor Fuel Counter (RRFC) system. ${ }^{10}$ 
The RRFC contains twelve ${ }^{3} \mathrm{He}$ tubes, each with its own preamplifier, polyethylene neutron moderator, and lead gamma-ray shielding; two AmLi interrogation sources; and an ion chamber in a sealed, stainless steel housing. MTR assemblies are loaded from the top; a funnel aids in the loading process. The fuel assembly is centered in the measurement cavity by baskets designed to fit each specific type of MTR assembly.

Calibration parameters for the RRFC were derived by calculation for each separate type of spent fuel assembly. Calibration by calculation, rather than by measurement, is necessary because of the lack of suitably characterized MTR-type spent-fuel assembly standards. However, the RRFC can be calibrated for fresh fuel by measuring adequately characterized fresh-fuel assemblies, thereby providing a benchmark for the spent-fuel calculations.

The calculational model was validated by measurements made on a fresh MTR test assembly that can be configured with various numbers of fuel plates in various geometries. The fitted calibration curve is

$$
R=a m /(1+b m)
$$

The calibration parameters obtained from the measurements and calculations agreed to within $1 \%$. The facility is now using the RRFC with the calculated calibration parameters for the spent MTR fuel.

\section{Use of Calorimetry/Gamma-Ray Spectroscopy to Reduce the Need for De- structive Analysis}

The combination of calorimetry and plutonium gamma-ray spectrometry is a powerful approach to characterizing plutonium-bearing WRMs. The precision and bias are not as good as chemical analysis, but the cost is considerably less and the results are much more timely. With sufficient counting time, precision and bias of better than $0.3 \%$ can be achieved for homogeneous and relatively pure plutonium samples. This is certainly sufficient for waste assay systems where the precision and bias are in the several percent range. The precision and bias are probably adequate for certifying secondary WRMs. In addition, in the US the calorimetry exchange program has been ongoing since 1981. The data from this program provide assurance of the accuracy of the approach at several facilities.

The combination of calorimetry and plutonium gamma-ray spectrometry should certainly be used for the reverification of standards on a scheduled basis.

\section{References}

1. H. O. Menlove, J. E. Stewart, S. Z. Qiao, T. R. Wenz, and G. P. D. Verrechia, "Neutron Collar Calibration and Evaluation for Assay of LWR Fuel Assemblies Containing Burnable Neutron Absorbers," Los Alamos National Laboratory report LA-11965-MS (ISPO-323) (November 1990).

2. H. O. Menlove, "Description and Operation Manual for the Active Well Coincidence Counter," Los Alamos Scientific Laboratory report LA-7823-M (ISPO-66) (May 1979). 
3. H. O. Menlove and J. E. Swansen, "A High-Performance Neutron Time-Correlation Counter," Nucl. Technol. 711, 497-505 (November 1985).

4. H. O. Menlove and J. E. Stewart, "A New Method of Calibration and Normalization for Neutron Detector Families," Los Alamos National Laboratory report LA-11229-MS (ISPO287) (April 1988).

5. H. O. Menlove, O. R. Holbrooks, and A. Ramalho, "Inventory Sample Coincidence Counter Manual," Los Alamos National Laboratory report LA-9544-M (ISPO-181) (November 1982).

6. N. Ensslin, "A Simple Self-Multiplication Correction for In-Plant Use," in Proc. 7th ESARDA Symposium on Safeguards and Nucl. Mater. Manage., (ESARDA, Ispra, Italy, 1985), ESARDA 19, pp. 223-238.

7. J. E. Stewart, R. R. Ferran, S. M. Simmonds, and H. O. Menlove, "Calibration Parameters from Monte Carlo Simulations for Neutron Coincidence Assay of MOX Fuel Elements-A Substitute for Physical Standards?," in Proc. ESARDA 11th Symposium on Safeguards and Nucl. Mater. Manage., (ESARDA, Ispra, Italy, 1989), ESARDA 22, pp. 135-141.

8. J. E. Stewart, "A Hybrid Monte Carlo/Analytical Model of Neutron Coincidence Counting," Trans. Am. Nucl. Soc. 53 149-151 (1986).

9. T. R. Wenz, H. O. Menlove, G. Walton and J. Baca, "Design and Calibration of the AWCC for Measuring Uranium Hexafluoride," Los Alamos National Laboratory report LA-12992 (ISPO-377) (August 1995).

10. M. E. Abhold, S.-T. Hsue, H. O. Menlove, G. Walton and S. Holt, "The Design and Performance of the Research Reactor Fuel Counter," Nucl. Mater. Manage. XXV (CDROM), 424-429 (1996). 


\section{QUALITY ASSURANCE OF STANDARDS}

\section{A. Calorimetry Exchange Program}

NDA performance may be tested by means of interlaboratory measurements on multiple standards produced from one well-characterized lot of material. An equal quantity of the reference material is placed in each container to be distributed: one to each participating facility. Measurements can then be made using the same type of NDA system: neutron, gamma/x-ray, or calorimeter. Interlaboratory exchange programs are useful in evaluating measurement biases between sites. The measurements made by each facility provide a basis for evaluating shipperreceiver $(\mathbf{S} / \mathrm{R})$ differences. The statistical information provided by an interlaboratory program can be used to evaluate whether a $S / R$ difference is statistically significant. A systematic difference in measurement results exchanged between two sites may presage a difference in $S / R$ results for a shipment of SNM measured by the same instruments. The known bias between facilities could be corrected using the exchange data. The standard can also be used in the facility for other measurement control purposes. The exchange program may indicate a statistically significant measurement bias for one laboratory compared to the consensus results and this may indicate a measurement problem.

The CALEX Program was initiated in 1981. The format of the calorimetry exchange program was similar in concept to other chemical exchange programs such as the NBL's Safeguards Analytical Laboratory Evaluation (SALE). For the CALEX program, plutonium (6\% ${ }^{240} \mathrm{Pu}$ ) oxide samples were prepared from a homogeneous batch of material. The elemental and isotopic compositions of the material were determined by chemical and mass spectrometric methods traceable to the NBS. The isotopic composition of the material used to prepare the samples is shown in Table XX. The results in Table XX for the $6 \%{ }^{240} \mathrm{Pu}$ (CALEX1) are based on measurements made by four analytical laboratories.

Table XX

Isotopic Composition of CALEX samples $\left(6 \%\right.$ and $\left.12 \%{ }^{240} \mathrm{Pu}\right) * 1,2$

\begin{tabular}{|c|c|c|c|}
\hline Isotope & $\begin{array}{c}\text { CALEX1 } \\
\text { (Weight \%) }\end{array}$ & $\begin{array}{c}\text { CALEX1 } \\
\text { RSD(\%) }\end{array}$ & $\begin{array}{c}\text { CALEX2 } \\
\text { (Weight \%) }\end{array}$ \\
\hline${ }^{238} \mathrm{Pu}$ & 0.00944 & 0.6 & 0.0853 \\
\hline${ }^{239} \mathrm{Pu}$ & 93.8676 & 0.003 & 86.5304 \\
\hline${ }^{240} \mathrm{Pu}$ & 5.86 & 0.06 & 12.169 \\
\hline${ }^{241} \mathrm{Pu}$ & 0.2338 & 0.57 & 1.0085 \\
\hline${ }^{242} \mathrm{Pu}$ & 0.0291 & 5.0 & 0.2067 \\
\hline${ }^{241} \mathrm{Am}$ & 0.1429 & 0.4 & 0.4320 \\
\hline
\end{tabular}

*CALEX1 isotopic compositions were measured on 1/1/89. Uncertainties are calculated from the data from the four reporting laboratories. CALEX2 isotopic compositions were measured on 7/11/95 with results from one reporting laboratory. 
One container with $400 \mathrm{~g}$ of plutonium was distributed to each participating DOE laboratory. Each site measured its standard with calorimetry and gamma-ray spectroscopy and reported the results to Mound Laboratory on a quarterly basis. There the data from all the laboratories was summarized and a quarterly report issued to the participants. The data were intended for use by participants in measurement control programs or to determine the bias of the measurement or both. No attempt was made to standardize operational procedures or the frequency of measurements. The quarterly average calorimetry results for the CALEX program in 1990 are shown in Fig. 32. ${ }^{1}$ The reported power measurements were decayed to a common date using plutonium and ${ }^{241} \mathrm{Am}$ half lives and the original mass spectrometry/alpha counting analyses of the materials lot. Most calorimetry results agreed to within $0.2 \%$ of the power, $0.9759 \mathrm{~W}$, predicted from the original plutonium weight and mass/alpha spectrometry measurements. The results for CALEX gamma-ray isotopic measurements are presented elsewhere in this guide.

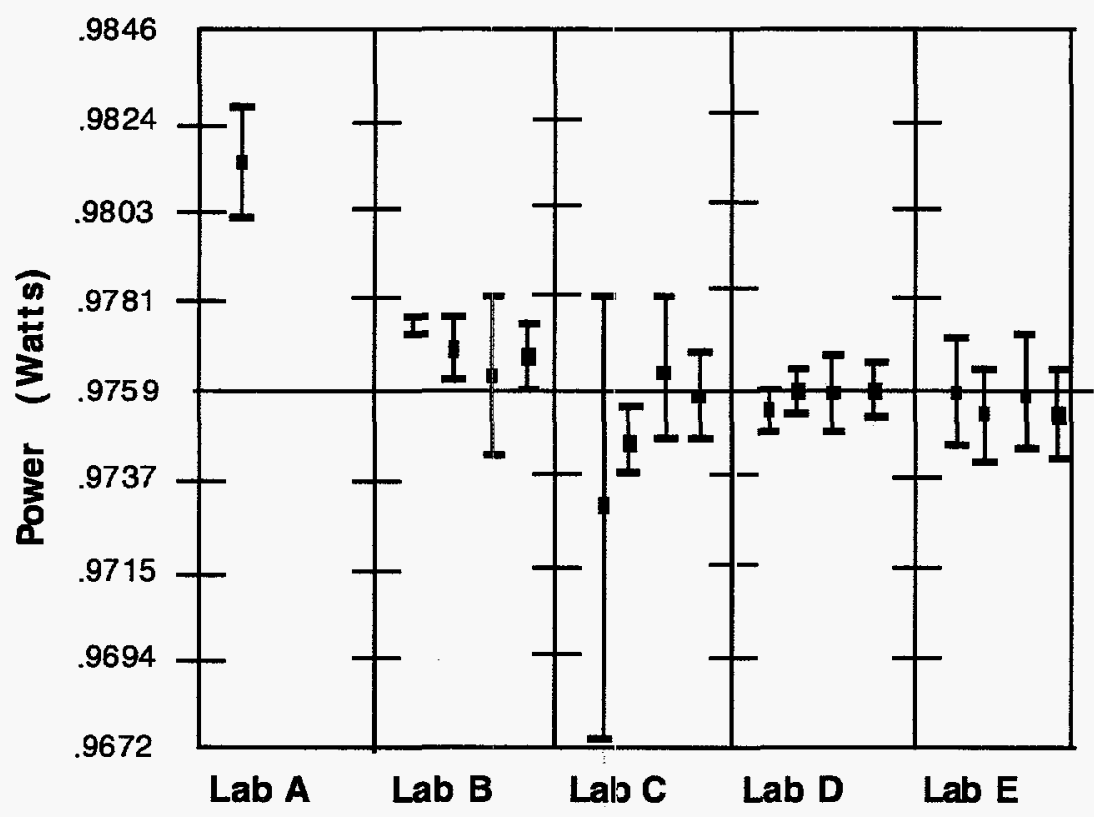

Fig. 32. 1990 interlaboratory calorimeter measurements of CALEX1 samples.

Recently the scope of the CALEX program was expanded. A series of well-characterized $12 \%{ }^{240} \mathrm{Pu}$ standards each weighing $2000 \mathrm{~g}$ with a thermal power of $6.2 \mathrm{~W}$ has been prepared. These samples provide higher wattage standards and isotopic compositions representative of higher burnup plutonium. ${ }^{2}$ The isotopic composition of this newer reference material (CALEX2) is shown in Table XX. The CALEX isotopic results are discussed elsewhere in this guide. The CALEX program is now administered by the NBL at ANL.

\section{B . Calibration Using Calorimetry/Gamma-Ray Assay}

Calorimeters are designed to capture all the heat flow from a plutonium sample. The temperature-sensing element completely surrounds the sides of the cylindrical sample chamber. Insulators and heat shunts at the top and bottom of the calorimeter ensure that the heat flowing 
through these areas is directed through the heat-sensing element. The heat distribution error can be determined by moving a small, compact ${ }^{238} \mathrm{Pu}$ heat source in the larger sample chamber and measuring the difference in instrument response for different locations. It has been found that this error is small, less than $0.4 \%$ for a 12 -in. diameter calorimeter. This insensitivity to position means that the heat measurement is independent of the location of the plutonium in the sample chamber. Thus, spatial inhomogeneities will not significantly affect the heat measurement result. The thermal power $W$ measured by heat-flow calorimeters is insensitive to thermal physical properties such as specific heat and the thermal conductivity of the material in the sample chamber. Only the time to reach thermal equilibrium is affected by these properties. The independence of calorimetry from material properties is its primary advantage over other more rapid, but matrixdependent, NDA techniques. A calorimeter will measure the same quantity of thermal power independent of how the SNM is distributed in the matrix and independent of the chemical or physical nature of the matrix. The same thermal power result will be obtained whether the SNM sample is solid, liquid, or gas, whether the SNM is pure material; whether the SNM is mixed with high-Z materials such as lead or depleted uranium, low-Z materials such as paper, oil, or plastic; or whether the SNM is mixed with scrap metal parts, such as from contaminated glove boxes or machine tools.

High-resolution gamma-ray spectroscopy using germanium detectors is associated with calorimetry measurements of plutonium-bearing materials. This type of measurement is needed to determine the isotopic composition from which the effective specific power of the sample, $P_{\text {eff }}$, is calculated. The quantity of plutonium in the sample is calculated from $W / P_{\text {eff }}$. The sample needs to be isotopically homogeneous so that the isotopic composition measured by gamma-ray spectroscopy represents the isotopic composition of the whole sample.

The relative independence of the calorimeter output with respect to matrix type indicates that calorimetry may be used in producing NDA physical standards. In general, physical standards for NDA instruments have been produced synthetically, by mixing known quantities of pure, wellcharacterized SNM with a matrix material similar in characteristics to the unknowns to be measured or by selecting representative containers from the material category in question and analyzing the contents using chemical methods. The chemical analysis may involve sampling the container contents or completely dissolving the container contents after the appropriate NDA measurement is made. If sampling is performed, a sampling error will be added to the overall standard uncertainty. These methods of producing physical standards are expensive because of the cost of chemical analyses and the generation of nuclear waste. A new standard set may cost more than $\$ 150,000$.

The chemical route to preparing standards can be bypassed in many cases by using calorimetry/gamma-ray measurements. The insensitivity of calorimetry to matrix and SNM distribution inside the sample container suggests that it could be used in preparing NDA standards at a production facility. Representative containers would be withdrawn from a population of items from one category and assayed by calorimetry/gamma-ray spectroscopy. These assayed items would serve as standards for other, more rapid, NDA methods such as neutron counting. The calorimetry/gamma-ray assay technique is traceable to NIST thus providing traceability for the new secondary standards. The method depends on the isotopic homogeneity of the material category to be measured. The assay technique would also be applicable to material categories where there are too many items to measure by calorimetry alone and where the more rapid NDA methods are needed. A transportable calorimeter could be moved to locations with no permanent calorimeter setup. 
The calorimetry/gamma-ray standards preparation technique has not been generally used to produce secondary standards for NDA instrumentation but has been used for a closely related activity: verification measurements. From 1972 to 1995, calorimetry and gamma-ray measurements were performed by Mound Laboratory teams for the Safeguards Branch of the DOE Albuquerque Operations Office. Once a year these teams would visit other DOE facilities to verify the plutonium content of selected items in conjunction with DOE audits. A portable water bath calorimeter and germanium counting system were shipped to the sites from Mound for the measurements. The site visits lasted two weeks including setup time. In 1983, a third measurement technique, passive TNCC was added. ${ }^{3}$ This technique was used to increase the number of samples that could be evaluated in a limited time.

The method by which the three techniques were used for audit verification campaigns was essentially equivalent to the calorimetry standards preparation technique. Items to be measured were selected from different material categories by the auditors. Some plutonium categories measured were product oxide, low-fired oxide, electrorefining salt residue, direct oxide-reduction salt, and direct oxide-reduction metal. Within each category, the Mound team selected several items that spanned the plutonium mass range of the material category in question. Calorimetry and gamma-ray spectroscopy were used to determine the plutonium and the effective ${ }^{240} \mathrm{Pu}$ mass of these items. The new standards were then used to determine the calibration curve of the neutron counter. An in-field calibration curve, including additional calorimetry measurements, is shown for an audit verification in Fig. 33. ${ }^{4}$ The remaining samples of the category were then measured by the neutron counter using this calibration curve. Certain material categories were difficult to assay by this technique. Materials with known isotopic inhomogeneities such as crucible pieces or anode heels in matrices with high levels of americium fluoride salt presented difficulties for the gammaray isotopic analysis. ${ }^{4}$ However, for most material types, the calorimetry/gamma-ray standardization technique worked satisfactorily.

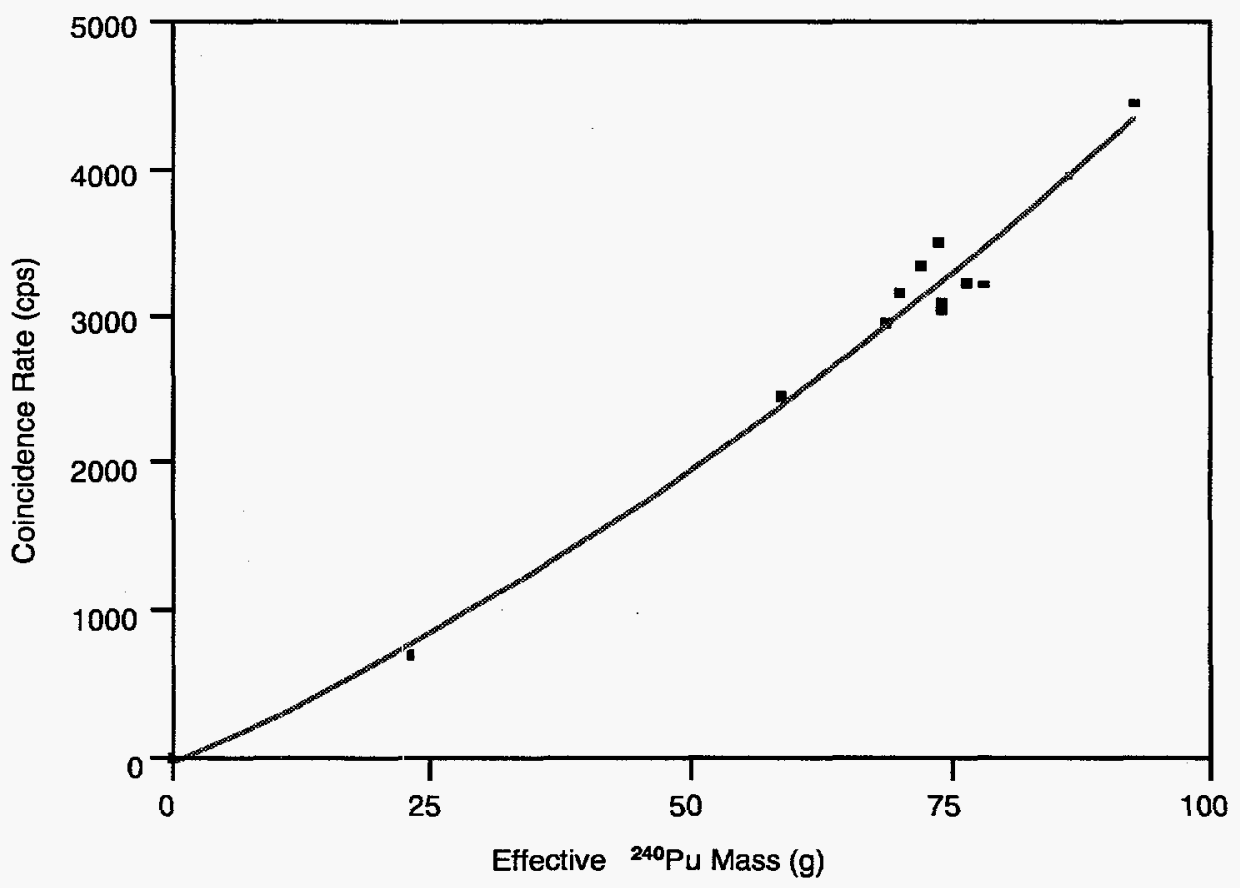

Figure 33. In-field calibration via calorimetric/gamma-ray assay for passive TNCC. 5 
The calorimeter verification measurements of plutonium materials provide an example of using calorimetry and gamma-ray isotopic measurements to create secondary NDA standards. The procedure outlined above could be applied by an individual facility to the assay of its own inventory and would remove the need to buy, prepare, or chemically characterize NDA physical standards.

\section{Standards Recertification}

NDA standards contain uranium- or plutonium-bearing materials similar in quantity and isotopic composition to the materials to be assayed. The matrix material in which the SNM is located is chosen to represent the material category. In that case, the matrix material of the standard affects the NDA measurement the same way as the matrix affects the sample measurement.

Although the standard may have been synthesized or characterized correctly, over time changes in physical properties could make the standard no longer representative of the material category measured. The changes could lead to a drift in the response of the NDA instrument to the standard. Changes in physical properties include stratification of particle size or clumping of particles or both. Gamma-ray tomographic techniques could be used to detect shifts in physical properties that might affect NDA instrument performance. Grow-in of gamma-emitting progeny with high gamma branching fractions, such as ${ }^{241} \mathrm{Am}$ from ${ }^{241} \mathrm{Pu}$ or ${ }^{208} \mathrm{Tl}$ from ${ }^{232} \mathrm{U}$, can degrade the performance of gamma-ray analyses. The properties of the standard could vary over time and no longer represent the material category. New standards may have to be produced to replace the old ones.

Physical standards may exist with inexactly known isotopic compositions. For short-lived isotopes the imprecision of the isotopic measurement can lead to a larger uncertainty in isotopic composition over a period of years. This uncertainty is a concern for highly accurate calorimeter measurements and leads to the need to recalibrate ${ }^{238} \mathrm{Pu}$ standards. Currently there is no DOE complex-wide requirement concerning the frequency of recertification of NDA standards. However, the Albuquerque Field Office (DOE/AFO) has set a maximum calibration interval of 5 years for any calibrations in support of weapons, nonweapons, or general operations activities. ${ }^{5}$ The maximum period can be exceeded if formal documentation is provided justifying a longer interval. This DOE order has been applied to setting the 5-year maximum interval for the calibration of ${ }^{238} \mathrm{Pu}$ heat sources.

The recertification of ${ }^{238} \mathrm{Pu}$ heat standards is an example of the need for recertification because of the change in standard properties with time. Plutonium-238 heat sources are certified to a high accuracy, $0.015 \%(=150 \mathrm{ppm})$. Uncertainties in the initial isotopic measurements of the

${ }^{238} \mathrm{Pu}$ source material, partly caused by the lack of suitable isotopic reference materials, lead to an uncertainty in the decay correction used to calculate the thermal power of the heat standard at later times. Table XXI compares the power predicted from isotopic decay from initial value measured by mass spectrometry with the average power measured in the calibration calorimeter for a typical 1-W heat standard over a 19-year period. The data indicate an overall $90 \mathrm{ppm}$ drift over the time range. A linear least-squares fit can be performed on the changing bias; the uncertainty of that fit can be used to estimate the standard accuracy over an extrapolated time period. The accuracy is estimated by lbiasl +12 sigma fitl.

The heat sources must be recalibrated every 3 to 5 years. Recalibrations previously carried out for the DOE complex at Mound are now done at Los Alamos using special calibration calorimeters. The calorimetry calibration procedure is described elsewhere in this guide. 
Table XXI

Calibration and Recalibration Measurements for ${ }^{238} \mathrm{Pu}$ Heat Standard $1.0 \mathrm{~W}$

\begin{tabular}{|c|c|c|c|}
\hline $\begin{array}{c}\text { Date } \\
\text { (days) }\left[^{*}\right]\end{array}$ & $\begin{array}{c}\text { Power(observed) } \\
\left.\text { (Watts) }{ }^{* *}\right]\end{array}$ & $\begin{array}{c}\text { Power(Predicted) } \\
\text { (Watts) }\end{array}$ & $\begin{array}{c}\text { Power (observed) - } \\
\text { Power (Predicted) } \\
\text { (Watts) }\end{array}$ \\
\hline 3001 & 0.972683 & 0.972638 & 0.000045 \\
\hline 3001 & 0.972682 & 0.972638 & 0.000044 \\
\hline 4800 & 0.935921 & 0.935888 & 0.000033 \\
\hline 6185 & 0.908499 & 0.908517 & -0.000018 \\
\hline 7475 & 0.883717 & 0.883727 & -0.000010 \\
\hline 9887 & 0.839101 & 0.839137 & -0.000036 \\
\hline 9884 & 0.839153 & 0.839191 & -0.000038 \\
\hline
\end{tabular}

[*] Day $1=$ January 1,1966

[**] Data are averages of replicate (4 to 9 ) calorimeter measurements.

\section{References}

1. T. Barnett, "Calorimetry Exchange Program, Annual Data Report for 1990," EG\&G Mound Laboratories report MLM-MU-91-63-005 (1991).

2. S. M. Long, "Fabrication of $12 \%$ Pu-240 Calorimetry Standards," Nucl. Mater. Manage. XXIV (Proc. Issue), 348-351(1995).

3. D. A. Rakel, "In-Field Calibration of Neutron Correlation Counter Via Calorimetry and High Count Rate Gamma-Ray Isotopic Abundance Measurements," IAEA-SM-293/126, Nuclear Safeguards Technology 1986, Volume 2, 239-249, IAEA, 1987.

4. L. J. Satkowiak, J. A. McDaniel, and D. P. Renz, "In-Field Calibration of a Neutron Correlation Counter Via Calorimetric Assay," 2nd International Workshop on Calorimetric Assay, Santa Fe, New Mexico, 27 October 1994.

5. "Standards and Calibration Program, Appendix A - General Requirements," DOE Albuquerque Operations Office Order AL-57XA/AA Rev 2. 


\section{RECOMMENDATIONS}

Following are six recommendations based on issues we identified in preparing this report. We also give the rationale for each recommendation.

1. Establish, within the United States, a resource center (such as PERLA in the EEC) housing an extensive collection of well-characterized $N D A$ standards and laboratories in which NDA systems are characterized, calibrated, and used for training.

The US has no dedicated center where users can bring their NDA instruments for evaluation and calibration. We recommend establishing, in the US, a resource center such as PERLA in the EEC. The purposes of this US center would be as follows.

a. The user community could validate their NDA instruments and simulation techniques using the wide range of types and masses of standards available at this center. The standards at the center must all be well-characterized.

b. Fully calibrating the majority of neutron NDA instruments for bulk SNM requires a set of relatively large plutonium- or uranium-bearing standards or both for each material type. Many of the developed neutron NDA instruments have been transferred to the commercial sector and, therefore, some standard instrument models are widely available. Each instrument from a given family of a standard model should have the same calibration curve shape. The "universal" calibration curve of a family of counters could carefully be established for a given material type and reference detector at this center. Also, the user could normalize the response of the individual counter to the reference detector(s) by using the universal curve and one or a few CRMs or WRMs.

c. The well-characterized standards would further serve the user community in a variety of NDA workshops and training courses.

\section{Request that NBL/IRMM develop several CRMs.}

Several CRMs needed by the user community should be produced. Because of the difficulty in preparing CRMs, they should be selected carefully in conjunction with the development effort to reduce the number of standards required to calibrate NDA systems. If NBL and the IRMM have difficulty in fabricating some of these CRMs, national laboratories can provide support. The plutonium-bearing CRMs may be issued in limited sets because of the difficulty in shipping. The two following needs stand out:

a. Plutonium metal standards. These will be used to normalize the response of individual counters and can use the universal calibration curve established for these families of instruments. We find that 3-5 thin disks of pure plutonium metal in the mass range 0.5 to $10 \mathrm{~g}$ would be very useful. 
b. Low-density standards. These are useful in many ways. They can be used to calibrate SGS systerns, which are designed to assay low-density wastes. Because of the relatively low density of these standards, the self-attenuation can be easily corrected and these standards can be used for holdup calibration and testing. One to two standards of plutonium and uranium in the mass range of 10 to $50 \mathrm{~g}$ would be adequate. Only a small number of CRMs are required because the SGS calibration is linear as a function of SNM mass.

\section{Develop bulk-plutonium WRMs}

Several WRMs needed by the user community at the resource facility should be produced. These items should not be shipped from site to site. Three needs have been identified.

a. Impure oxide set. Develop a set of impure plutonium oxide standards to be used in studying the effects of impurities on neutron coincidence and multiplicity counting. Six standards will be made; each will have a mass of $1 \mathrm{~kg}$ and a constant isotopic composition $\left(\sim 6 \%{ }^{240} \mathrm{Pu}\right)$. The impurities and their $(\alpha, \mathrm{n})$ yields relative to spontaneous fission (the alpha value) will be as follows:

1. none,

2. silicon with $\alpha=1$,

3. silicon with $\alpha=1.5$,

4. fluorine with $\alpha=2$,

5. boron with $\alpha=2.5$, and

6. magnesium with $\alpha=3$.

b. Pure plutonium metal set. Develop a set of pure plutonium metal standards to study the effects of neutron multiplication on neutron coincidence and multiplicity counting. Six standards will be made in the form of right circular cylinders and constant isotopic composition $\left(\sim 6 \%{ }^{240} \mathrm{Pu}\right)$. The plutonium masses will be 30,60 , $125,250,500$, and $1000 \mathrm{~g}$.

c. MOX and wet plutonium oxide set - Develop a set of three wet oxide standards and a set of three MOX standards to use in studying the effects of moisture and induced fissions in uranium on neutron coincidence and multiplicity counting. The wet oxide standards will contain $1 \mathrm{~kg}$ of plutonium with constant isotopic composition $\left(\sim 6 \%{ }^{240} \mathrm{Pu}\right)$. The moisture contents will be $1 \%, 2 \%$, and $3 \%$ by weight. The MOX standards will have the same plutonium isotopic composition, will contain natural uranium, and will all have a combined uranium and plutonium mass of $1 \mathrm{~kg}$. The plutonium masses will be 100,200 , and $300 \mathrm{~g}$. 


\section{Establish the combination of calorimetry and plutonium gamma spectrometry as an acceptable, routine method to certify plutonium- bearing WRMs.}

The combination of calorimetry and plutonium gamma spectrometry is a powerful approach for characterizing plutonium-bearing WRMs. The combination is less precise and has a larger bias than chemical analysis, but the combination is cheaper and more timely. With sufficient counting time, precision and bias can be better than $0.3 \%$ for homogeneous and relatively pure plutonium samples. This is certainly sufficient for waste assay systems where the precision and bias are in the several percent range and probably adequate for certifying secondary WRMs. In addition, in the US the calorimetry exchange program has existed since 1981. Data from this program assure accuracy and precision of the approach at several facilities.

The combination of calorimetry and plutonium gamma spectrometry should certainly be used for the scheduled reverification of standards in measurement control procedures.

\section{Publish a reference manual on the standards (RMs) that cannot and should not be shipped from site to site.}

Some NDA standards, because of physical size, cannot be shipped from site to site. Also, some low-level waste standards that are used to flag disposable waste at the 100 $\mathrm{nCi} / \mathrm{g}$ level should not be shipped to other sites because of ease of preparation. It will be very useful to publish a practical guide to illustrate how these standards are made.

\section{Establish the calculational approach as an acceptable, routine method of NDA instrument calibration.}

Some standards cannot be characterized without being partially or completely destroyed, for example, spent reactor fuel-elements. These standards would also be difficult to store and maintain. For spent-fuel assay systems, a credible calculational method should be developed, which must be acceptable to the regulatory agency. An example of this approach is the calculation of fresh fuel assemblies and comparison to the measured response, which can serve as a validation step in the calculational standard approach.

Also, for fresh plutonium-bearing reactor fuel assemblies, standards are difficult and expensive to access for calibration. Using fast-reactor fuel-element fabrication data, calibration curves based on calculations have been shown to reproduce passive coincidence measurements to within $\sim 1 \%$ over a wide range of element types.

In fact, for any plutonium item that is thoroughly characterized with regard to geometry, mass, composition, and matrix, calculational approaches should yield calibration parameters to within $1 \%$. This should be verified case by case, to develop standard calculational methods. 


\section{APPENDIX A}

\section{BIBLIOGRAPHY}

\section{A. Gamma-Ray Based NDA}

a. "American National Standard for Nuclear Materials-Calibration Material for Nondestructive Assay Systems That Count Passive Gamma-Rays-Guide to Preparing," American National Standards Institute, Inc. report N15.35-1983.

b. J. L. Parker, "The Use of Calibration Standards and the Correction for Sample Self-Attenuation in Gamma-Ray Nondestructive Assay," Los Alamos National Laboratory report LA-10045, Rev. (1986).

\section{Enrichment Measurements}

a. J. L. Parker and M. Brooks, "Accurate, Wide-Range Uranium Enrichment Measurements by Gamma-Ray Spectroscopy: I. Characterization of Enrichment Standards, II. Demonstration of Enrichment Measurement Capability," Los Alamos National Laboratory report LA-11277-MS (September 1988).

b. P. Matussak, "Accurate Determination of the ${ }^{235} \mathrm{U}$ Isotope Abundance by Gamma Spectrometry: A User's Manual for the Certified Reference Material EC-NRM171/NBS-SRM-969," Institute für Kernphysik report KfK 3752, Kernforschungszentrum, Karlsruhe, Federal Republic of Germany (1985).

c. W. De Bolle, G. Müschenborn, and P. De Bièvre, "The Potential of Double Collector $\mathrm{UF}_{6}$ Mass Spectrometry for Verification of Isotopic Homogeneity. Application to $\mathrm{U}_{3} \mathrm{O}_{8}$ Rms for Gamma Spectrometry," in Proc. 7th ESARDA Symposium on Safeguards and Nucl. Mater. Manage. (ESARDA, Ispra, Italy, 1985), ESARDA 19, pp. 277-286.

\section{Densitometry}

a. W. J. McGonnagle, M. K. Holland, S. Scott Reynolds, N. M. Trahey, and A. C. Zook, "Evaluation and Calibration of a Los Alamos National Laboratory $\mathrm{L}_{\mathrm{III}}$-Edge Densitometer," New Brunswick Laboratory report NBL-307 (July 1983).

\section{Segmented Gamma Scanning}

a. S. -T. Hsue, S. M. Simmonds, V. L. Longmire, and S. M. Long, "Design and Fabrication of SGS Plutonium Standards," in Fourth International Conference on Facility Operations-Safeguards Interface (American Nuclear Society, La Grange Park, Illinois, 1991), pp. 410-413.

b. S.-T. Hsue, S. M. Simmonds, V. L. Longmire, and S. M. Long, "Design and Fabrication of SGS Plutonium Can Standards," Los Alamos National Laboratory, Safeguards Assay Group report N-1/WP-90-7 (1990).

c. J. K. Sprinkle, R. N. Likes, J. L. Parker, and H. A. Smith, "Reference Materials for Nondestructive Assay of Special Nuclear Material. Volume 1: Uranium Oxide 
Plus Graphic Powder, Vol. 1," Los Alamos National Laboratory report LA-9910MS, Vol. 1 (October 1983).

d. F. Hsue, S. M. Long, S. -T. Hsue, and M. C. Miller, "Design and Fabrication of the Uranium Drum Standards," Nucl. Mater Manage. XXII (Proc. Issue), pp. 940-944 (1993).

e. S. M. Long, D. J. Martinez, S. C. Serna, and V. L. Longmire, "Fabrication of Plutonium/Diatomaceous Earth Standards," Nucl. Mater. Manage. XIX (Proc. Issue), pp. 226-228 (1990).

f. T. E. Sampson, "Bias in Segmented Gamma Scans Arising from Size Differences Between Calibration Standards and Assay Samples," Nucl. Mater. Manage. X X (Proc. Issue), pp. 235-239 (1991).

\section{Small U Standards}

a. J. K. Sprinkle, R. N. Likes, and H. A. Smith, "Reference Materials for Nondestructive Assay of Special Nuclear Material. Volume 2: Thin Metal Foils of Highly Enriched Uranium, Vol. 2," Los Alamos National Laboratory report LA9910-MS, Vol. 2 (October 1983).

\section{B . Neutron based NDA}

\section{208-L Drums}

a. R. Dierckx, L. Bondar, R. Bosser, W. B. Bremner, P. Cresti, P. Filss, J. W. Leake, H. Ottmar, C. Vicini, and H. Wurz, "European Interlaboratory Test Measurements on Alpha-Contaminated Waste," Nucl. Sci. \& Technol., CEC report EUR-12609-EN (1990).

b. P. M. Rinard, E. L. Adams, H. O. Menlove, and J. K. Sprinkle, Jr., "The Nondestructive Assay of 55-Gallon Drums Containing Uranium and Transuranic Waste Using Passive-Active Shufflers," Los Alamos National Laboratory report LA-12446-MS (November 1992).

c. S. M. Long, F. Hsue, C. W. Hoth, R. Fernandez, C. Bjork, and J. K. Sprinkle, "Design and Fabrication of 55-Gallon Drum Shuffler Standards," Nucl. Mater. Manage. XXIII (Proc. Issue), pp. 470-473 (1994).

\section{Small Containers of Pu}

a. S. Guardini, G. Guzzi, F. Mousty, S. Baumann, E. Kuhn, and P. De Regge, "Quality Control Provisions Applied in the Preparation and Characterization of $\mathrm{Pu}$ Bearing PERLA Standards," Institute for Safety Technol., PERLA Safeguards Performance Laboratory, CEC report EUR-13038-EN (1990).

b. S. Guardini, G. Guzzi, F. Mousty, C. Bigliocca, E. Kuhn, N. Doubek, R. Fiedler, A. Zoigner, S. Baumann, K. H. Nelges, G. Hesbacher, P. De Regge, L. Vandevelde, R. Boden, and D. Huys, "Pu PERLA Standards: Quality Control Provisions During Preparation and Analyses of the Samples," in Proc. 7th ESARDA Symposium on Safeguards and Nucl. Mater. Manage. (ESARDA, Ispra, Italy, 1985), ESARDA 19, p. 543. 
c. S. Guardini and J. K. Sprinkle, Jr., "The Preparation of Large Standards for NDA Measurements," Nucl. Mater. Manage. XX (Proc. Issue), pp. $995-997$ (1991).

\section{Calorimetry}

a. S. M. Long, "Update on Fabrication of 12\% PU-240 Calorimetry Standards," Los Alamos National Laboratory document LA-UR-94-3517.

\section{Other References}

a. S.-T. Hsue and T. Marks, "Measurement Control Program for NDA Instruments," in Proc. ANS/INMM Conf. Safeguards Technol.: Process Safeguards Interface, Hilton Head Island, South Carolina, November 28-December 2, 1983 (US DOE/New Brunswick Laboratory, August 1984), CONF-831106, pp. 422-425.

b. P. A. Russo, R. B. Strittmatter, E. L. Sandford, I. W. Jeter, E. McCullough, and G. L. Bowers, "Operation of Automated NDA Instruments for In-Line HEU Accounting at Y-12," in Proc. ANS/INMM Conf. Safeguards Technol.: Process Safeguards Interface, Hilton Head Island, South Carolina, November 28December 2, 1983 (US DOE/New Brunswick Laboratory, August 1984), CONF831106, pp. 112-134.

c. J. E. Stewart, R. R. Ferran, S. M. Simmonds, and H. O. Menlove, "Calibration Parameters from Monte Carlo Simulations for Neutron Coincidence Assay of MOX Fuel Elements-A Substitute for Physical Standards?," in Proc. 11th ESARDA Symposium on Safeguards and Nucl. Mater. Manage., (ESARDA, Ispra, Italy, 1989), ESARDA 22, pp. 135-141.

d. G. R. Waterbury, D. C. Croley, and C. F. Metz, "Variability in the Standardization of Ceric Sulfate Solution for the Titration of Plutonium," Los Alamos Scientific Laboratory report LA-3841 (1967).

e. R. H. Augustson and T. D. Reilly, "Fundamentals of Passive Nondestructive Assay of Fissionable Material," Los Alamos Scientific Laboratory report LA-5651M (1974).

f. S. M. Long, "Design and Fabrication of NDA Standards," in Proceedings of the Plutonium/Uranium Recovery Operations Conference, Knoxville, TN, October 1720, 1994, Los Alamos National Laboratory document LA-UR-94-3496.

g. G. E. Bentley, V. T. Hamilton, E. J. Peterson, and L. E. Wangen, "The Selection and Evaluation of Internal Standard Elements Based on Elemental/Instrumental Covariance," in Proceedings of the Applied Spectroscopy, Vol. 40, pp. 949-953 (1986).

h. E. S. Gladney, P. E. Neifert, and N. W. Bower, "Determination of Silicon in National Institute of Standards and Technology Biological Standard Reference Materials by Instrumental Epithermal Neutron Activation and X-Ray Fluorescence Spectrometry," in Proceedings of the Analytical Chemistry, Vol. 61, pp. 18341836 (1989).

i. S. L. Yarbro, S. L. Dunn, and S. B. Schreiber, "Preparation of Pure Neptunium Oxide for Nondestructive Assay Standards," Los Alamos National Laboratory report LA-11890 (1991). 
j. G. E. Bosler, "Analytical Techniques to Reduce the Number of Physical Standards Needed for Neutron Coincidence Counters," Los Alamos National Laboratory report LA-8509-MS (1981).

k. L. A. Bruckner, W. L. Delvin, and M. W. Hume, "On Precision and Accuracy (Bias) Statements for Measurement Procedures," Los Alamos National Laboratory report LA-11190-MS, (January 1988). 